\title{
Strong Higgs interactions at a linear collider
}

\author{
Roberto Contino $^{a}$ Christophe Grojean, ${ }^{b, c}$ Duccio Pappadopulo ${ }^{d, e}$ Riccardo Rattazzi $^{f}$ \\ and Andrea Thamm ${ }^{c, f}$ \\ ${ }^{a}$ Dipartimento di Fisica, Università di Roma "La Sapienza" and INFN - Sezione di Roma, \\ Roma, Italy \\ ${ }^{b}$ ICREA at IFAE, Universitat Autònoma de Barcelona, \\ E-08193 Bellaterra, Spain \\ ${ }^{c}$ CERN, Physics Department, Theory Unit, \\ Geneva, Switzerland \\ ${ }^{d}$ Department of Physics, University of California, \\ Berkeley, CA 94720, U.S.A. \\ e Theoretical Physics Group, Lawrence Berkeley National Laboratory, \\ Berkeley, CA 94720, U.S.A. \\ ${ }^{f}$ Institut de Théorie des Phénomènes Physiques, EPFL, \\ CH-1015 Lausanne, Switzerland \\ E-mail: roberto.contino@roma1.infn.it, christophe.grojean@cern.ch, \\ pappadopulo@berkeley.edu, riccardo.rattazzi@epfl.ch, \\ andrea.thamm@epfl.ch
}

ABSTRACT: We study the impact of Higgs precision measurements at a high-energy and high-luminosity linear electron positron collider, such as CLIC or the ILC, on the parameter space of a strongly interacting Higgs boson. Some combination of anomalous couplings are already tightly constrained by current fits to electroweak observables. However, even small deviations in the cross sections of single and double Higgs production, or the mere detection of a triple Higgs final state, can help establish whether it is a composite state and whether or not it emerges as a pseudo-Nambu-Goldstone boson from an underlying broken symmetry. We obtain an estimate of the ILC and CLIC sensitivities on the anomalous Higgs couplings from a study of $W W$ scattering and $h h$ production which can be translated into a sensitivity on the compositeness scale $4 \pi f$, or equivalently on the degree of compositeness $\xi=v^{2} / f^{2}$. We summarize the current experimental constraints, from electroweak data and direct resonance searches, and the expected reach of the LHC and CLIC on $\xi$ and on the scale of the new resonances.

Keywords: Higgs Physics, Beyond Standard Model, Technicolor and Composite Models ARXIV EPRINT: 1309.7038 


\section{Contents}

1 Introduction 1

2 General parametrization of the Higgs couplings 3

3 Current constraints on the Higgs couplings $\quad 7$

3.1 EW precision observables

3.2 Direct coupling measurements 9

3.3 Resonance searches 9

4 What can be learned from single and double Higgs production? 11

5 What can be learned from triple Higgs production? 14

$\begin{array}{lll}5.1 & \text { Symmetry structure } & 14\end{array}$

$\begin{array}{ll}5.2 \text { Quantitative analysis of } V V \rightarrow h h h & 15\end{array}$

6 Quantitative analysis of $V V \rightarrow V V$ and $V V \rightarrow h h \quad 19$

$\begin{array}{lll}6.1 & \text { Identification cuts } & 19\end{array}$

$\begin{array}{ll}6.2 V V \rightarrow V V \text { scattering } & 20\end{array}$

$6.3 V V \rightarrow h h$ scattering $\quad 23$

$\begin{array}{lll}6.4 & \text { Double Higgs-strahlung } & 29\end{array}$

$\begin{array}{lll}7 & \text { Discussion } & 35\end{array}$

A Dimension-8 operators for strong scatterings $\quad 39$

B Double Higgs-strahlung 40

\section{Introduction}

With the discovery of the Higgs boson [1,2] and with the absence, thus far, of any clear evidence for New Physics (NP), a basic feature of the dynamics underlying electroweak symmetry breaking (EWSB) has begun to materialize: the new states associated with that dynamics do not seem to be as light as naturalness considerations would have recommended. That this might be the case has been suggested for quite some time by considerations based on precision electroweak and flavor data, but the LHC results have made this picture more concrete. Of course, we may just sit at the edge of New Physics and evidence may plentifully show up in the next run of the LHC. However, once a bit of un-naturalness is accepted, it is natural to expect, or fear, that history may repeat itself at $13 \mathrm{TeV}$. For instance, keeping the composite Higgs scenario in mind [3-9], a plausible situation is one where, at the end of its program, the LHC will have only measured inconclusive $O(10 \%)$ deviations 
from the Standard Model (SM) in the Higgs couplings. In a definitely more optimistic situation these small deviations would appear along with some new states, but without a clear indication for their role in the EWSB dynamics. Under the above circumstances, the next experimental project would be more one of exploration and discovery than one of refinement and consolidation. With this in mind, a high-energy hadron machine like the LHC at $\sqrt{s}=33 \mathrm{TeV}[10-12]$ would superficially seem better suited than a cleaner but less powerful leptonic machine like the ILC [13] or CLIC [14-16]. However, given the criticality of the decisions we may face in the coming years, it is important to carefully assess the potential of each machine in each plausible NP scenario. It is the goal of this paper to provide one such assessment: by focussing on a high-energy lepton machine such as CLIC, we shall explore its potential in the exploration of the composite Higgs scenario.

Single Higgs production at a linear collider, even at $500 \mathrm{GeV}$, is known to be a very sensitive probe of compositeness [17], even though an indirect one. In the case where resonances are still out of reach, a more direct probe on compositeness is offered by the study of the interactions of longitudinally polarized electroweak vector bosons and the Higgs. The relevant processes are $V V \rightarrow V V$ and $V V \rightarrow h h(V=W, Z)$, whose cross sections grow like $E^{2}$. There exist several studies of $V V \rightarrow V V$ at hadron machines [18$28]$ but just a few of $V V \rightarrow h h$ [28, 29]. The study of these processes in hadron collisions is not an easy task. $V V \rightarrow V V$ offers final states with leptons that stand out well against the QCD background. However, the genuine SM contribution to $V V \rightarrow V V$ happens to be numerically so large that the effects of compositeness dominate only at very high energy, where LHC parton luminosities are small [28]. The result is a poor reach on the scale of compositeness. In the case of $V V \rightarrow h h$ the genuine SM contribution is numerically small, so that in principle this would be a good probe of compositeness. However the final states and branching ratios of Higgs decay are not favorable in a hadronic environment. The reach on compositeness from this process at the LHC is thus also not very good. Instead, as we will show in this paper, a machine like CLIC offers the right combination of a clean environment and center-of-mass energy to significantly probe the composite Higgs scenario. The improvement compared to hadron machines is particularly stark for $V V \rightarrow h h$. Our main result is that $V V \rightarrow h h$ at CLIC offers about half the reach on the scale of compositeness as single Higgs production at a $500 \mathrm{GeV}$ ILC. However the observation of a cross section for $h h$ production that grows with energy would be a more direct and convincing evidence of the strongly coupled nature of NP. It also turns out that in the presence of a signal in $V V \rightarrow h h$ one may in principle be able to make more refined statements about the nature of $h$. First of all, via a comparison with single $h$ processes, one can nicely and directly confirm that $h$ is part of a doublet. Moreover one can in principle test whether $h$ is a pseudo Nambu-Goldstone boson (PNGB) living in a coset space [3-9] or whether it is a generic composite scalar. A way in which this could also be done is by studying triple Higgs production: $V V \rightarrow h h h$. This process could be marginally observable in the case of a generic composite $h$, while in the case of a PNGB non-trivial selection rules suppress its rate below observability.

This paper is organized as follows. In section 2, we review the general parametrization of the Higgs couplings and the relative importance of energy-growing $2 \rightarrow 2$ scattering 
processes. Current constraints on the couplings, especially from electroweak precision tests, and their consequences on the scale of NP are discussed in detail in section 3 . In sections 4 and 5, we study the information contained in single, double and triple Higgs production on the structure of the underlying theory. A quantitative analysis of the ILC and CLIC sensitivities on the anomalous couplings and their reach in the parameter space is given in section 6 . We present our conclusions and outlook in section 7 .

\section{General parametrization of the Higgs couplings}

Under the assumption that the mass scale at which new states appear is large, $m_{\rho} \gg m_{h}$, the recently discovered Higgs boson can be described by means of an effective Lagrangian. Motivated by the experimental evidence accumulated both at LEP and recently at the LHC, we will assume that the dynamics behind electroweak symmetry breaking has an approximate custodial invariance, under which $h$ is a singlet. The effective Lagrangian can be organized by expanding in the number of derivatives and classifying the various terms according to the number of $h$ fields. The expression obtained in this way extends the EW chiral Lagrangian [30-32] to include the light state $h$. Such a construction does not assume that $h$ is part of an $\mathrm{SU}(2)_{L}$ doublet, nor does it make hypotheses on the strength of its interactions, as long as it is weakly coupled at energies of the order of its mass. It is thus completely general and applies as well to the case where $h$ is a Higgs-like impostor not directly involved in EWSB. Despite the strong indications from the experimental measurements in favor of a SM-like Higgs boson, hence in favor of the Higgs being part of a weak doublet, the purpose of this paper is to study processes with multiple Higgs production, and the general parametrization adopted can help emphasizing the peculiarity of a linearlyrealized EW symmetry. In the following we will further assume that $h$ is a $\mathrm{CP}$-even scalar. This choice is both motivated from the theoretical point of view (it follows for example in minimal composite Higgs theories), and supported by the preliminary results on the Higgs couplings obtained by the LHC collaborations.

At $O\left(p^{2}\right)$ in the derivative expansion, the bosonic part of the effective Lagrangian thus reads $[28,33-36]$

$$
\mathcal{L}=\frac{1}{2}\left(\partial_{\mu} h\right)^{2}-V(h)+\frac{v^{2}}{4} \operatorname{Tr}\left(D_{\mu} \Sigma^{\dagger} D^{\mu} \Sigma\right)\left(1+2 a \frac{h}{v}+b \frac{h^{2}}{v^{2}}+b_{3} \frac{h^{3}}{v^{3}}+\ldots\right),
$$

where the $2 \times 2$ matrix $\Sigma$ is defined as

$$
\Sigma(x)=\exp \left(i \sigma^{a} \chi^{a}(x) / v\right)
$$

and $\chi^{a}(x)$ are the Nambu-Goldstone (NG) bosons of the global coset $\mathrm{SO}(4) / \mathrm{SO}(3)$ which are eaten in the unitary gauge to form the longitudinal polarizations of the $W$ and $Z$. Under the custodial $\mathrm{SO}(3)$ the $\chi$ 's transform as a triplet. In eq. $(2.1), V(h)$ is the potential for $h$

$$
V(h)=\frac{1}{2} m_{h}^{2} h^{2}+d_{3}\left(\frac{m_{h}^{2}}{2 v}\right) h^{3}+d_{4}\left(\frac{m_{h}^{2}}{8 v^{2}}\right) h^{4}+\ldots,
$$


and $a, b, b_{3}, d_{3}, d_{4}$ are arbitrary dimensionless parameters. The dots stand for terms of higher order in $h$. For the SM Higgs $a=b=d_{3}=d_{4}=1$ while all higher-order terms vanish. The dilaton couplings are instead characterized by the relations $a=b^{2}, b_{3}=0$ [37-42].

Any deviation of the couplings $a, b, b_{3}$ from their SM values implies the energy growth of some scattering amplitude whose strength can be parametrized in terms of a "running" coupling $\bar{g}(\sqrt{s})$ at a given center-of-mass (c.o.m.) energy $\sqrt{s}$. For example, the couplings $a$ and $b$ control the strength of the interactions in $2 \rightarrow 2$ processes among $\chi$ 's and $h$. Under the assumption of $\mathrm{SO}(3)$ custodial invariance, the scattering amplitudes read

$$
\begin{aligned}
\mathcal{A}\left(\chi^{a} \chi^{b} \rightarrow \chi^{c} \chi^{d}\right) & =A(s, t, u) \delta^{a b} \delta^{c d}+A(t, s, u) \delta^{a c} \delta^{b d}+A(u, t, s) \delta^{a d} \delta^{b c}, \\
\mathcal{A}\left(\chi^{a} \chi^{b} \rightarrow h h\right) & =A_{h h}(s, t, u) \delta^{a b}, \\
\mathcal{A}\left(\chi^{a} \chi^{b} \rightarrow \chi^{c} h\right) & =A_{h \chi}(s, t, u) \epsilon^{a b c},
\end{aligned}
$$

where $s, t, u$ are the usual Mandelstam variables. As implied by the equivalence theorem [43, 44 , at high energy each of the above amplitudes equals one in which each external $\chi$ is replaced by the corresponding longitudinal vector boson $\left(\chi^{ \pm} \rightarrow W_{L}^{ \pm}, \chi^{0} \rightarrow Z_{L}\right)$. From the Lagrangian in eq. (2.1), at leading order in the derivative expansion, it follows $A(s, t, u)=$ $\left(1-a^{2}\right) s / v^{2}$ and $A_{h h}(s, t, u)=\left(a^{2}-b\right) s / v^{2}$. In both these cases the scattering amplitude defines a coupling strength

$$
\mathcal{A}(2 \rightarrow 2)=\delta_{h h} \frac{s}{v^{2}} \equiv(\bar{g}(\sqrt{s}))^{2},
$$

where we indicate by $\delta_{h h}$ both $a^{2}-1$ (for $\chi \chi \rightarrow \chi \chi$ ) and $a^{2}-b$ (for $\chi \chi \rightarrow h h$ ). A measurement of the $V_{L} V_{L} \rightarrow V_{L} V_{L}$ and $V_{L} V_{L} \rightarrow h h(V=W, Z)$ scattering rates at a given center of mass energy $\sqrt{s}$ thus corresponds to the measurement of an effective coupling $\bar{g}(\sqrt{s})$, characterizing the strength of the EWSB dynamics. The effective coupling $\bar{g}(\sqrt{s})$ grows with energy so that perturbativity, and with it the validity of the effective Lagrangian, would be lost at the scale $\sqrt{s_{*}}$ where $\bar{g}\left(\sqrt{s_{*}}\right) \sim 4 \pi$. A reasonable expectation is then that new states will UV complete the effective Lagrangian at a scale $m_{\rho} \leq \sqrt{s_{*}}$. The new states would expectedly saturate the growth of the effective coupling to $g_{\rho} \equiv \bar{g}\left(m_{\rho}\right) \leq 4 \pi$.

In the case of the scattering $\chi \chi \rightarrow \chi h$, Bose and crossing symmetries imply that the function $A_{h \chi}(s, t, u)$ is antisymmetric under the exchange of any two Mandelstam variables. As a consequence, the lowest-order contribution to $A_{h \chi}(s, t, u)$ arises at $O\left(p^{6}\right)$, that is $A_{h \chi} \propto(s-u)(u-t)(t-s)$, in accordance with the fact that there exists no local operator at the level of two and four derivatives giving a vertex with three $\chi$ 's. The corresponding scattering amplitude, $V_{L} V_{L} \rightarrow V_{L} h$, is expected to be suppressed by a factor $\left(s / m_{\rho}^{2}\right)^{2}$ compared to that of $V_{L} V_{L} \rightarrow V_{L} V_{L}, h h$, and is thus not a sensitive probe of the Higgs interaction strength at energies below the scale $m_{\rho}$ of New Physics. In fact, the absence of an energy growth in the $\chi \chi \rightarrow \chi h$ amplitude could have been anticipated on the basis of a simple symmetry argument. The request of custodial invariance fixes the global coset to be $\mathrm{SO}(4) / \mathrm{SO}(3)$, which is a symmetric space. The grading of its algebra, under which all 
broken generators and thus all NG bosons change sign, is an accidental symmetry of the $O\left(p^{2}\right)$ Lagrangian $(2.1)^{1}$

$$
P_{L R}: \quad \chi^{a}(x) \rightarrow-\chi^{a}(x), \quad h(x) \rightarrow h(x) .
$$

Any process with an odd number of $\chi$ 's, including $\chi \chi \rightarrow \chi h$, must thus vanish at leading derivative order. Furthermore, although $P_{L R}$ is generically broken at $O\left(p^{4}\right)$, it turns out that none of the $P_{L R}$-odd operators with four derivatives contributes to $2 \rightarrow 2$ processes [45]. In absence of custodial symmetry, on the other hand, the global coset is $\mathrm{SU}(2) \times \mathrm{U}(1) / \mathrm{U}(1)$ rather than $\mathrm{SO}(4) / \mathrm{SO}(3)$. This is not a symmetric space, and there is no grading symmetry which forbids vertices with three NG bosons at $O\left(p^{2}\right)$. In particular, the operator $\left[\operatorname{Tr}\left(\Sigma^{\dagger} D_{\mu} \Sigma \sigma^{3}\right)\right]^{2} h$ contains the term $h \partial_{\mu} \chi^{3}\left(\chi^{+} i \overleftrightarrow{\partial_{\mu}} \chi^{-}\right)$, which gives $\mathcal{A}\left(\chi^{+} \chi^{-} \rightarrow \chi^{3} h\right) \propto(t-u)$. In practice, the experimental results on the Higgs couplings obtained by the LHC collaborations already set tight limits on possible custodial breaking effects [46-48] and thus on the energy growth of $V_{L} V_{L} \rightarrow V_{L} h$. These new constraints are not surprising given the very strong constraint on custodial symmetry breaking provided by electroweak precision tests at LEP/SLC/Tevatron.

One might ask whether the amplitude of the process $V_{T} V_{L} \rightarrow V_{L} h$, with one transversely polarized vector boson, grows with the energy and thus probes the Higgs interaction strength. By virtue of the equivalence theorem, at high energy this coincides with the amplitude of $V_{T} \chi \rightarrow \chi h$, for which a naive power counting would suggest $\mathcal{A} \sim g \sqrt{s} / v$. A direct calculation, on the other hand, reveals that the energy-growing term cancels after summing all relevant diagrams, thus implying $\mathcal{A}\left(V_{T} \chi \rightarrow \chi h\right) \sim g^{3}(v / \sqrt{s}) .{ }^{2}$ Eventually, the leading contribution to $V V \rightarrow V h$ comes from the scattering amplitude with two transversely polarized vector bosons, $\mathcal{A}\left(V_{T} V_{T} \rightarrow V_{L} h\right) \sim g^{2}$, which makes it clear that this process cannot be used to probe the Higgs interaction strength. Incidentally, notice that there is no analog cancellation in the scatterings with zero or two Higgses and one transverse vector boson, that is: $\mathcal{A}\left(V_{T} \chi \rightarrow h h\right) \sim\left(a^{2}-b\right) g \sqrt{s} / v$ and $\mathcal{A}\left(V_{T} \chi \rightarrow \chi \chi\right) \sim\left(a^{2}-1\right) g \sqrt{s} / v$.

So far our discussion has been general, since the effective Lagrangian (2.1) applies to any scalar $h$ with arbitrary couplings (provided the custodial symmetry is exact). One might however consider a situation in which future experiments constrain the Higgs couplings to be close to their SM value, so that only small deviations are allowed. In this case it is convenient to adopt a more specific effective description in which $h$ is assumed to be part of an $\mathrm{SU}(2)_{L}$ doublet $H$ and the Lagrangian is expanded in powers of the $H$ field (as well as in the number of derivatives). The list of dimension-6 operators of such an effective Lagrangian has been discussed at length in the literature [49-58], for recent reviews see refs. $[59,60]$. The case of a strongly interacting light Higgs (SILH) was addressed in this context in ref. [29], where a power counting was introduced to estimate the Wilson coefficients. A similar scenario, limited to bosonic operators, was also studied earlier in ref. [17]. A simple yet crucial observation is that any additional power of $H$ costs

\footnotetext{
${ }^{1}$ It coincides with parity up to a spatial inversion: $P=P_{0} P_{L R}$, with $P_{0}:\{\vec{x} \rightarrow-\vec{x}, t \rightarrow t\}$.

${ }^{2}$ The cancellation follows from the fact that all the diagrams have the same dependence on the Higgs couplings, namely they are all proportional to $a$. Since in the SM limit $a=1$ the amplitude cannot grow with the energy, by continuity this implies that the same holds true for any $a$.
} 
a factor $g_{\rho} / m_{\rho} \equiv 1 / f$, where $g_{\rho} \leq 4 \pi$ denotes the coupling strength of the Higgs to New Physics states; any additional derivative instead is suppressed by a factor $1 / m_{\rho}{ }^{3}$. If the light Higgs interacts strongly with the new dynamics, $g_{\rho} \gg 1$, then the leading corrections to low-energy observables arise from operators with extra powers of $H$ rather than derivatives. This remark greatly simplifies the list of relevant operators. By concentrating on the bosonic part of the SILH Lagrangian and assuming custodial invariance, there are two such operators involving only the Higgs multiplet

$$
O_{H}=\frac{c_{H}}{2 f^{2}} \partial_{\mu}|H|^{2} \partial^{\mu}|H|^{2}, \quad O_{6}=-\frac{c_{6} \lambda}{f^{2}}\left(H^{\dagger} H\right)^{3},
$$

where $\lambda$ is the quartic coupling which appears in front of the marginal operator $\left(H^{\dagger} H\right)^{2}$. The $O(1)$ coefficients $c_{H}$ and $c_{6}$ control the Higgs couplings $a, b, b_{3}, d_{3}$ at order $(v / f)^{2}$. Under the assumption of $h$ being part of a doublet, the Higgs couplings of eq. (2.1) are thus correlated and functions of a smaller set of parameters. Note that there exists only one dimension-6 structure involving fermions: $\left(H^{\dagger} H\right) H \bar{f} f$. Under the assumption of minimal flavor violation there are thus three additional operator coefficients $c_{t}, c_{b}$ and $c_{\tau}$, describing the non-linear couplings of the Higgs multiplet to up- and down-type quarks and to charged leptons respectively [29]. Operators of dimension 8 induce corrections of order $(v / f)^{4}$ to the Higgs couplings. As described in more detail in appendix A, there are only two dimension- 8 operators which modify $a, b, b_{3}, d_{3}$

$$
O_{H}^{\prime}=\frac{c_{H}^{\prime}}{2 f^{4}}|H|^{2} \partial_{\mu}|H|^{2} \partial^{\mu}|H|^{2}, \quad O_{8}=-\frac{c_{8} \lambda}{f^{4}}\left(H^{\dagger} H\right)^{4}
$$

where the coefficients $c_{H}^{\prime}$ and $c_{8}$ are expected to be of order 1 . The expressions for the couplings at $O\left(v^{4} / f^{4}\right)$ thus read

$$
\begin{aligned}
a & =1-\frac{c_{H}}{2} \frac{v^{2}}{f^{2}}+\left(\frac{3 c_{H}^{2}}{8}-\frac{c_{H}^{\prime}}{4}\right) \frac{v^{4}}{f^{4}}, \quad b=1-2 c_{H} \frac{v^{2}}{f^{2}}+\left(3 c_{H}^{2}-\frac{3 c_{H}^{\prime}}{2}\right) \frac{v^{4}}{f^{4}}, \\
b_{3} & =-\frac{4 c_{H}}{3} \frac{v^{2}}{f^{2}}+\left(\frac{14 c_{H}^{2}}{3}-2 c_{H}^{\prime}\right) \frac{v^{4}}{f^{4}}, \\
d_{3} & =1+\left(c_{6}-\frac{3 c_{H}}{2}\right) \frac{v^{2}}{f^{2}}+\left(\frac{15 c_{H}^{2}}{8}-\frac{5 c_{H}^{\prime}}{4}-\frac{c_{6} c_{H}}{2}-\frac{3 c_{6}^{2}}{2}+2 c_{8}\right) \frac{v^{4}}{f^{4}} .
\end{aligned}
$$

In the special case in which the Higgs doublet $H$ is a PNGB of a global breaking $\mathcal{G} / \mathcal{H}$, all powers $(H / f)^{n}$ can be resummed exactly by imposing $\mathcal{G}$ invariance. In this case $f$ must be identified with the decay constant of the NG bosons. For example, there are just two custodially symmetric cosets yielding only one complex doublet of Goldstones: $\mathrm{SO}(5) / \mathrm{SO}(4)$ and $\mathrm{SO}(4,1) / \mathrm{SO}(4)$. They lead to

$$
a=\sqrt{1-\xi}, \quad b=1-2 \xi, \quad b_{3}=-\frac{4}{3} \xi \sqrt{1-\xi},
$$

where $\xi=v^{2} / f^{2}>0$ for $\mathrm{SO}(5) / \mathrm{SO}(4)$ and $\xi=-v^{2} / f^{2}<0$ for $\mathrm{SO}(4,1) / \mathrm{SO}(4)$. By expanding the above relations to $O\left(\xi^{2}\right)$ one re-obtains those of eq. (2.11) for $c_{H}=1, c_{H}^{\prime}=2$.

\footnotetext{
${ }^{3}$ Extra powers of the gauge fields are also suppressed by $1 / m_{\rho}$ as they can only appear through covariant derivatives.
} 
The expression for $d_{3}$ depends instead on the form of the Higgs potential, which is model dependent since it requires some explicit breaking of the Goldstone symmetry $\mathcal{G}$. For example, in the minimal models MCHM4 and MCHM5 of refs. [61, 62] one has

$$
\text { MCHM4 : } \quad d_{3}=\sqrt{1-\xi}, \quad \text { MCHM5 : } \quad d_{3}=\frac{1-2 \xi}{\sqrt{1-\xi}},
$$

from which it follows $c_{6}=1, c_{8}=5 / 4$ in the MCHM 4 and $c_{6}=c_{8}=0$ in the MCHM5.

The fact that at $O\left(v^{2} / f^{2}\right)$ the couplings $a, b, b_{3}$ are affected by only one operator [29], whose coefficient $c_{H}$ can be always redefined away by a proper redefinition of $f$ (for example it can be set to 1), has an important consequence. Since the predictions of any coset $\mathcal{G} / \mathcal{H}$ must match those of the SILH Lagrangian at low energy, this implies that the expressions of eq. (2.11) are universal at first order in $v^{2} / f^{2}$, i.e. they are the same for a PNGB and for a generic scalar. At order $v^{4} / f^{4}$, instead, the couplings $a, b, b_{3}$ are modified by two operators, whose coefficients are thus related by a specific relation for any given coset $\mathcal{G} / \mathcal{H}$; for example, the coset $\mathrm{SO}(5) / \mathrm{SO}(4)$ implies $c_{H}^{\prime}=2 c_{H}$. One can thus distinguish the case of a generic SILH, where $c_{H}^{\prime}$ can have any value, from that of a PNGB Higgs.

\section{Current constraints on the Higgs couplings}

Past and current experiments set important constraints on the Higgs couplings and on the scale of New Physics $m_{\rho}$. In particular, the coupling $a$ is indirectly constrained by the precision tests of the EW observables performed at LEP, SLD and Tevatron, and directly measured in single Higgs processes studied at the LHC. However, there is currently no constraint on the couplings $b$ and $d_{3}$ as these can be measured only through double Higgs processes.

\subsection{EW precision observables}

The sensitivity of the EW observables on $a$ arises at the 1-loop level only through the Higgs contribution to vector boson self energies. ${ }^{4}$ This is the leading effect, two-loop corrections are small and thus negligible. Compared to a few years ago, the information that comes from the EW fit has sharpened considerably [63-66]. This is mainly due to the value of the Higgs mass being now precisely known experimentally, so that a global fit can be used to extract the Higgs coupling to vector bosons directly, but also due to the new and more precise measurement of the $W$ mass from Tevatron. For example, compared to the average of Tevatron and LEP measurements $m_{W}=(80.425 \pm 0.034) \mathrm{GeV}$ used in the 2006 final report on EW tests at the $Z$ pole [67], the current world average $m_{W}=(80.385 \pm 0.015) \mathrm{GeV}[68]$ has an error smaller by more than a factor 2 . As a matter of fact, among the various observables sensitive to the Higgs coupling $a, m_{W}$ is the one which leads to the most precise determination [66]. Focusing on the 1-loop Higgs contribution to the vector boson self energies, the dependence on $a$ can be straightforwardly derived from that on $m_{H}$ at $a=1$ [69]: the $b$-quark forward-backward asymmetry $A_{F B}^{b}$ prefers values $a<1$, while the leptonic asymmetries $A_{l}$ and $m_{W}$ favor values slightly larger than

\footnotetext{
${ }^{4}$ The 1-loop Higgs contribution to the $b \bar{b} Z$ vertex is suppressed by $y_{b}^{2}$ and thus negligible.
} 
1. Overall, the global fit of $a$ is dominated by $m_{W}$ due to its small uncertainty, with the other observables individually playing a minor role. By using the results from the GFitter collaboration [63], we find that in absence of additional NP contributions to the EW fit, the Higgs coupling is expected to lie in the interval $0.98 \leq a^{2} \leq 1.12$ with $95 \%$ of probability. A similar result has been recently obtained by ref. [66]. This is an extremely strong bound which seems to disfavor Higgs compositeness as a natural solution of the little hierarchy problem, in particular its realizations through compact cosets where $a$ is always reduced compared to its SM value (see for example eq. (2.13)).

To better understand this result it is useful to perform a two-dimensional fit in terms of the Peskin-Takeuchi $\hat{S}$ and $\hat{T}$ parameters [70, 71]. It is well known that modifying the Higgs coupling to vector bosons compared to its SM prediction leads to a logarithmically divergent shift in these two parameters, with $\Delta \hat{S}>0$ and $\Delta \hat{T}<0$ [69]. For $m_{H}=125 \mathrm{GeV}$ and $a=1$ the theoretical point lies slightly outside the $68 \%$ contour, and by decreasing $a$ it moves further outside the experimentally preferred region, following a trajectory almost orthogonal to the probability isocontours. Thus, small reductions of the coupling $a$ have dramatic impact on the fit. Values $a>1$ are less constrained but also theoretically less motivated, as they require either non-compact cosets (like for example $\mathrm{SO}(4,1) / \mathrm{SO}(4)$ ), or a sizable tree-level contribution from a scalar resonance with isospin $I=2[72,73] .{ }^{5}$ If one excludes these more exotic theoretical scenarios, one concludes that sizable New Physics contributions to the EW observables, in particular to the vector boson self energies, are required to accommodate $\sim O(10 \%)$ shifts in the Higgs coupling $a$.

A negative and large $\Delta \hat{S}$ can follow from loops of fermion resonances [74-77]. A sizable and positive $\Delta \hat{T}$ could also be generated by the 1-loop exchange of composite fermions, in particular the top partners. For example, if both SM top chiralities couple with the same strength to the strong dynamics, one naively expects $\Delta \hat{T} \sim \xi y_{t}^{2} /\left(16 \pi^{2}\right)$ (see for example ref. [29]), which shows that it is possible to obtain $\Delta \hat{T} \sim$ a few $\times 10^{-3}$ for $\xi \sim O(10 \%)$. Corrections of this size would dramatically modify the range of $a$ preferred by the EW fit, especially if accompanied by an additional $\Delta \hat{S}<0$. For example, by assuming $\Delta \hat{T}=+1.5 \times 10^{-3}$ (with no extra $\Delta \hat{S}$ ), we find that the $95 \%$ interval on the Higgs coupling becomes $0.70 \leq a^{2} \leq 0.92$. It is thus interesting to see under what conditions models can accommodate such corrections while satisfying all other constraints, in particular on the $Z \bar{b} b$ vertex, and investigate what their predictions for the production of the top partners are at the LHC. The first analyses that appeared in the literature seemed to indicate a generic difficulty to obtain positive $\Delta \hat{T}[69,78-80]$. However, a more detailed exploration of the full parameter space in a broader class of models has shown that there is more freedom in accommodating a positive and sizeable $\Delta \hat{T}$ while keeping corrections to $Z \bar{b} b$ under control [76] (see also ref. [81]).

One might wonder if a modified value of $a$ can be helpful in relaxing the tension of the $b$-quark observables $A_{F B}^{b}$ and $R_{b} \cdot{ }^{6}$ In fact, as we already pointed out, all the

\footnotetext{
${ }^{5}$ The latter possibility can be directly tested experimentally, since the $I=2$ multiplet includes doublycharged scalars which can be produced and observed at the LHC.

${ }^{6}$ By including the two-loop calculation of $R_{b}$ performed by ref. [82], the pulls of $A_{F B}^{b}$ and $R_{b}$ are respectively $+2.7 \sigma$ and $-2.1 \sigma[66]$ (see also [63] for similar results).
} 
observables, including those related to the $b$ quark, depend on $a$ mainly through the 1-loop contribution of the Higgs to the vector boson self-energies. As a consequence, any NP correction to $a$ cannot lead to an effect restricted to the $b$-quark sector, but will propagate to all observables. Notice also that excluding $A_{F B}^{b}$ from the fit pushes $a$ towards larger values, which are even more problematic from the theoretical viewpoint, although the effect is small. It is thus clear that the existence of a tension in the $b$ observables does not lead to any room for relaxing the strong bound on the Higgs coupling $a$. Yet, the fact that in a fit to the couplings of $b_{L}$ and $b_{R}$ to the $Z$ the SM point lies outside the $95 \%$ probability contour (see refs. $[65,66,83]$ ) might indicate that the contribution from NP states is already at work.

Apart from possible New Physics effects, the fit of $a$ is strongly sensitive to the value of the $W$ and top quark masses. We have already stressed that $m_{W}$ dominates over the other observables. Its current experimental measurement is $\sim 1.2 \sigma$ larger than the one preferred by the EW fit, if it goes down in the future also the central value of $a$ will diminish. The strong dependence on the top mass originates from the 1-loop correction to the $\rho$ parameter, $\Delta \rho=\Delta \hat{T} \propto m_{t}^{2} G_{F}$, which we have seen has an important impact on $a$. In this regard one must notice that the error reported in the current Tevatron average $m_{t}=(173.18 \pm 0.94) \mathrm{GeV}[84]$ does not include the theoretical uncertainty on the definition of the parameter extracted from the event kinematics in terms of the $\overline{M S}$ mass. If one instead adopts the larger error $\sigma_{t}=2.8 \mathrm{GeV}$ that follows from measuring the $\overline{M S}$ mass directly from the $t \bar{t}$ cross section [85], one finds that the uncertainty on $a$ increases by a non-negligible amount [66]. While these issues have to be considered to make a precise determination of the Higgs coupling, the overall picture which emerges from the EW fit seems quite robust: $O(10 \%)$ shifts in $a$ require sizeable NP contributions to the vectorboson self energies.

\subsection{Direct coupling measurements}

The precision currently reached at the LHC on the direct measurement of the Higgs couplings to vector bosons and to fermions is, on the other hand, more limited. The exact value depends on the assumptions one makes to extract the couplings. For example, one can make a two-dimensional fit of $a$ and $c$, where the latter parametrizes a common rescaling of all the Yukawa couplings. Even neglecting the second solution at $c<0$, the uncertainty in the official fits of the ATLAS and CMS collaborations is of the order of $\sim 20 \%$ on $a$ and even larger on $c[47,48]$. In particular, while ATLAS prefers values $a>1$, the best fit value of CMS is for $a<1$. A naive combination of these results leads to a smaller uncertainty [86-91], but more data are definitely required to form a clearer picture. Preliminary studies indicate that eventually a precision of $\sim 5 \%$ on $a$ should be reached at the $14 \mathrm{TeV}$ LHC with an integrated luminosity of $300 \mathrm{fb}^{-1}[92,93]$.

\subsection{Resonance searches}

Searches for direct production of resonances at the LHC also set important constraints on the mass scale $m_{\rho}$ of a new strongly-interacting sector. Here we consider the case of a generic $\mathrm{SO}(5) / \mathrm{SO}(4)$ composite Higgs theory as a benchmark scenario, although the 


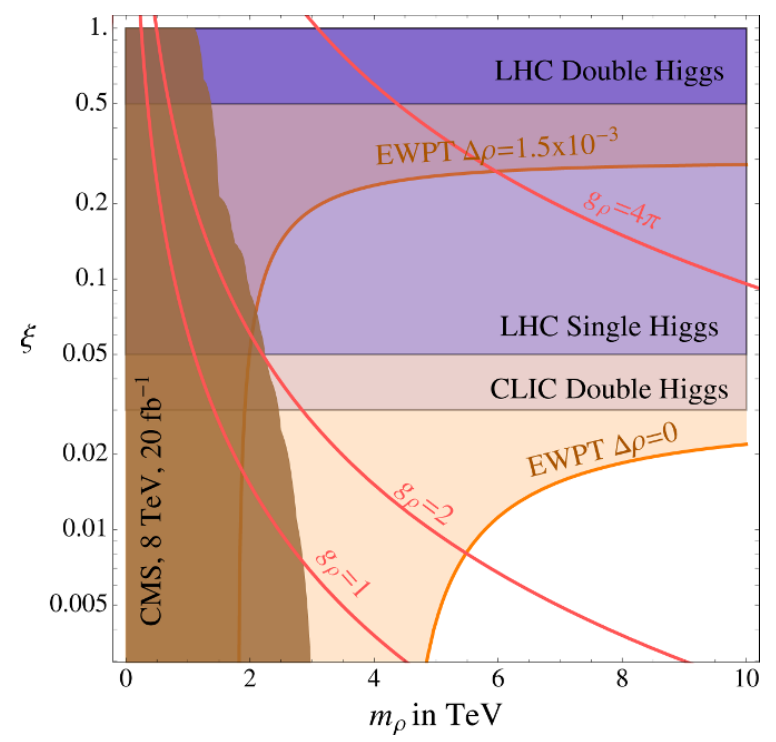

Figure 1. Summary of current constraints (orange curves and brown region) and expected sensitivities at CLIC and the LHC (horizontal regions) on $\xi=(v / f)^{2}$ and the mass of the lightest spin-1 resonance $m_{\rho}$ for $\mathrm{SO}(5) / \mathrm{SO}(4)$ composite Higgs theories. See text.

actual bounds will depend on the details of the strong dynamics and on how it couples to the SM fermions. For illustrative purposes we focus on the lightest spin-1 resonance of the strong sector, which we denote by $\rho$, and assume that it transforms as a $(3,1)$ under $\mathrm{SU}(2)_{L} \times \mathrm{SU}(2)_{R} \sim \mathrm{SO}(4)$ (for recent studies in this direction see for example [45, 9496]). The dominant production is via Drell-Yan processes (see for example ref. [97]). A class of theories motivated both theoretically and experimentally is one in which the spin-1 resonance couples to light fermions only through its mixing to the SM gauge fields [98] (see ref. [99] for alternative possibilities). In this case the Drell-Yan production cross section scales as $1 / g_{\rho}^{2}$, since couplings of the resonances to the SM fermions are suppressed by $1 / g_{\rho}$. The strongest exclusion limits are currently set by the LHC searches performed at $8 \mathrm{TeV}$ with $20 \mathrm{fb}^{-1}$ in final states with one lepton and missing transverse energy [100] or dileptons [101, 102], looking for charged and neutral spin-1 resonances respectively. For values of $\xi$ of order 1 , searches for resonances decaying into $W Z$, in particular those with three leptons in the final state [103, 104], give slightly stronger bounds. ${ }^{7}$ Assuming the $\rho$ to be a $(\mathbf{3}, \mathbf{1})$ of $\mathrm{SU}(2)_{L} \times \mathrm{SU}(2)_{R}$, we translated the bounds on $(\sigma \times B R)$ set by the experimental collaborations into a combined exclusion region in the $\left(\xi, m_{\rho}\right)$ plane.

The situation of direct and also indirect constraints is summarized in figure 1 for the case of a generic $\mathrm{SO}(5) / \mathrm{SO}(4)$ composite Higgs theory. We fix $a_{\rho} \equiv m_{\rho} /\left(g_{\rho} f\right)=1$, where we define $g_{\rho}$ as the physical coupling strength between three $\rho$ resonances. The fundamental free parameters of the new dynamics are then the mass of the spin- 1 resonance, $m_{\rho}$, and the strengths of the Higgs interactions parametrized by $\xi=\left(v^{2} / f^{2}\right)$. The dark brown region on the left shows the current $95 \%$ combined limit from direct production of the charged $\rho^{ \pm}$

\footnotetext{
${ }^{7}$ We find that the more recent searches for spin-1 resonances decaying to pairs of vector bosons with boosted decay products [105-107] give less strong constraints.
} 
at the LHC decaying to $l \nu$ and $W Z \rightarrow 3 l \nu$ final states. A similar exclusion region follows from the limits on the production of the neutral $\rho^{0}$. The dark (medium light) horizontal purple bands of figure 1 indicate instead the sensitivity on $\xi$ expected at the LHC from double (single) Higgs production with $300 \mathrm{fb}^{-1}$ of integrated luminosity (see footnote 18 for the definition of sensitivity adopted in this paper). The value shown for the case of double Higgs production is based on a naive (and perhaps optimistic) extrapolation of the study of ref. [28]; a more precise determination requires an updated analysis for $m_{h}=125 \mathrm{GeV}$. As we will discuss in section 6, the study of double Higgs processes alone at CLIC is expected to lead to a precision on $\xi$ larger than what obtainable at the LHC through single Higgs studies. In the plot of figure 1 this is illustrated by the lowest horizontal band. The possibility of directly testing such small values of $\xi$ at CLIC has to be compared with the indirect bounds set by the EW precision data. By including only the tree-level contribution $\Delta \hat{S}=m_{W}^{2} / m_{\rho}^{2}$ from the $\rho$ exchange [45] and the 1-loop IR effect from the modified Higgs couplings, we find that the region above the lower orange band is excluded at $95 \%$. For $m_{\rho} \rightarrow \infty$ the upper limit on $\xi$ tends to $\sim 0.02$, as previously reported in the discussion of the EW fit. In the absence of other contributions to the oblique parameters, masses $m_{\rho} \lesssim 5 \mathrm{TeV}$ are already excluded even for very small $\xi$. Lowering the value of $a_{\rho}$ makes the bound on $m_{\rho}$ weaker, but does not change much that on $\xi$. The allowed region instead opens up in presence of an additional contribution to $\hat{T}$ : for $0<\Delta \hat{T} \leq+1.5 \times 10^{-3}$ the $95 \%$ exclusion boundary varies between the upper and lower orange lines and masses as low as $m_{\rho} \sim 2 \mathrm{TeV}$ can still be viable. The domain of validity of our predictions, $g_{\rho}<4 \pi$, is below the upper red line.

\section{What can be learned from single and double Higgs production?}

In this section we discuss what could be learned directly, or indirectly, from a program of precise Higgs measurements at CLIC. For definiteness we can imagine a scenario where the LHC did not measure deviations from the SM larger than $O(20 \%)$ in single Higgs production, and also no clear indications emerged on what the underlying theory may be (new particles may have been discovered but not with a clear role, i.e. no supersymmetric particles). There are various broad questions one can in principle address with these measurements. One question is whether the scalar $h$ is elementary or composite. Other questions concern the nature of $h$, whether or not it fits into an SU(2) doublet (an explicit, if not well motivated, example of a non-doublet Higgs-like scalar is a dilaton [37-42]) or whether or not it is a PNGB. To some extent the information will be indirect, so it is worth illustrating the logic in some detail.

Consider the issue of compositeness first. Of course, in order to directly answer this question, it would be necessary to explore the energy scale associated with the new states and observe the onset of a novel UV regime, perhaps described by a strongly coupled CFT. That would be the analogue of observing the hadron to parton transition in QCD processes. However, the measurement of low-energy quantities can already give an appraisal of the strength of the underlying interactions, thus favoring or disfavoring a composite scenario. Indeed for an $\mathrm{SU}(2)$ Higgs doublet, a heavy particle with mass $m_{\rho}$ and coupling to the 
Higgs $g_{\rho}$ modifies the low-energy couplings by a relative amount of order $\left(g_{\rho} v / m_{\rho}\right)^{2}{ }^{8}$ For instance, massive fermions with a vectorlike mass $m_{\rho}$ and a Yukawa interaction to the Higgs of strength $g_{\rho}$ affect the coupling of $h$ to two gluons and two photons by a relative amount $\sim\left(g_{\rho} v / m_{\rho}\right)^{2}$ (for recent work in the context of composite Higgs models see e.g. ref. [111113]). Similarly, a heavy singlet scalar $S$ coupled to the Higgs doublet via a trilinear term $g_{\rho} m_{\rho} S|H|^{2}$ mixes by an angle $\theta \sim g_{\rho} v / m_{\rho}$ with $h$, implying shifts in its couplings of order $\theta^{2} \sim\left(g_{\rho} v / m_{\rho}\right)^{2}{ }^{9}$ In the absence of new states below a certain scale $M$, the observation of deviations of order $\delta_{h}^{e x p}$ in single Higgs production would then imply a qualitative lower bound on the coupling

$$
g_{\rho}>\sqrt{\delta_{h}^{e x p}} \frac{M}{v} .
$$

Sizeable deviations $\delta_{h}^{\text {exp }}$ in the absence of new states would suggest a strong coupling and, indirectly, $h$ compositeness. A more direct measurement of the strength of the underlying interaction is obtained by a study of the processes $W W \rightarrow W W$ and $W W \rightarrow h h$. As discussed in section 2, a deviation from $a=b=1$ leads to a cross section that grows with $s$. The $2 \rightarrow 2$ amplitude can be taken as a measure of a "running" coupling $\bar{g}(\sqrt{s})$, see eq. (2.7). The measurement of an enhancement, quantified by $\delta_{h h}^{e x p}$, in these processes at an energy $\sqrt{s}$, corresponds directly, though qualitatively, to a lower bound on the strength of the interaction

$$
g_{\rho}>\bar{g}(\sqrt{s}) \sim \sqrt{\delta_{h h}^{\exp }} \frac{\sqrt{s}}{v} .
$$

Equations (4.1) and (4.2) look similar, and not by chance. Notice, however, that the second equation corresponds to a direct measurement of the coupling, and is thus a more robust estimate. Indeed, at a precise machine such as CLIC a detailed study of $2 \rightarrow 2$ processes would allow even stronger conclusions. The point is that eq. (2.7) is only the leading term in a derivative expansion, the subleading corrections being of relative size $s / m_{\rho}^{2}$ :

$$
\mathcal{A}(2 \rightarrow 2)=\delta_{h h} \frac{s}{v^{2}}\left(1+O\left(\frac{s}{m_{\rho}^{2}}\right)\right)
$$

In principle at CLIC one could measure the leading $O(s)$ contribution and set an upper bound $\epsilon_{h h}$ on the relative size of the $O\left(s^{2}\right)$ term. That would indirectly suggest that there are no new states below a mass $M \sim \sqrt{s} / \sqrt{\epsilon_{h h}}$ and that the amplitude will keep rising at least until that scale. That would amount to a stronger indirect bound

$$
g_{\rho}>\bar{g}(M) \sim \sqrt{\frac{\delta_{h h}^{e x p}}{\epsilon_{h h}}} \frac{\sqrt{s}}{v} .
$$

We clearly see here the value of being able to measure $2 \rightarrow 2$ processes with high precision below the threshold of New Physics. Of course another possibility is that of directly observing, rather than setting limits on, the $O\left(s^{2}\right)$ effects from the tails of heavy resonances.

\footnotetext{
${ }^{8}$ Notice that a light Higgs mass $m_{h}=125 \mathrm{GeV}$ disfavors maximal values $g_{\rho} \sim 4 \pi$ unless some (additional) tuning is present in the Higgs potential. See for example refs. [108-110].

${ }^{9}$ One could also consider a potential $V=-m_{\rho}^{2}|S|^{2}+g_{\rho}^{2}|S|^{2}|H|^{2}+g_{\rho}^{2}|S|^{4}$, by which $\langle S\rangle \sim m_{\rho} / g_{\rho} \equiv f$, and reach the same conclusion.
} 
In this case detailed information on the strong dynamics, such as the quantum numbers of its resonances, can come from the comparison of different scattering channels, see for example refs. $[45,114-116] .{ }^{10}$

Consider now the properties of $h$ from the standpoint of symmetries. In the case of the SILH, in which $h$ fits into a doublet of $\mathrm{SU}(2)$ arising from some unspecified dynamics at the scale $m_{\rho}$, the bosonic couplings $a, b, b_{3}$ are predicted in terms of just one parameter at $O\left(v^{2} / f^{2}\right)$, as illustrated by eq. (2.11). In particular, by defining $\Delta a^{2} \equiv a^{2}-1$ and $\Delta b \equiv b-1$ one has

$$
\Delta b=2 \Delta a^{2}\left(1+O\left(\Delta a^{2}\right)\right),
$$

where the higher-order corrections are determined by the tower of higher-dimensional operators with two derivatives and $2 n H$ fields using the SILH power counting. Furthermore, in the very special case where $H$ is a PNGB the whole tower of operators and the resulting $W W h^{n}$ couplings are all fixed in terms of a single parameter $\xi$. Equation (2.13) reports for example the predictions of the minimal $\mathrm{SO}(5) / \mathrm{SO}(4)$ and $\mathrm{SO}(4,1) / \mathrm{SO}(4)$ theories. In both cases eq. (4.5) becomes exactly

$$
\Delta b=2 \Delta a^{2} .
$$

From single Higgs production one would be able to measure $\Delta a^{2}$ with an error $\sim 10^{-2}$, maybe of a few per mille $[13,17,123-126]$. The measurement of $W W \rightarrow h h$, as we will discuss in the next sections, allows one in principle to measure $\Delta b$ with an error of order $10^{-2}$. Equations (4.5) and (4.6) can then be tested at the percent level. For instance, in the case of a SILH not embedded in a coset one could imagine finding $\Delta a^{2}, \Delta b \gtrsim 0.1$ and to be compatible with eq. (4.5) but violating eq. (4.6) by an amount bigger than the expected percent accuracy. On the other hand, for $\Delta a^{2}, \Delta b<0.1$, it would not be possible to distinguish between a SILH and a PNGB. Finally, down to $\Delta a^{2}, \Delta b \sim 10^{-2}$ one could find that eq. (4.5) is not respected, indirectly speaking against the embedding of $h$ in a doublet. It should however be pointed out that such a scenario, normally associated with a fully composite $h$, would more probably imply $\Delta$ 's of order 1 , which are already excluded by the current LHC results. It should also be remarked that the only case of this type with some mild motivation is the one of a light dilaton, corresponding to

$$
\Delta b=\Delta a^{2},
$$

implying a vanishing contribution to $W W \rightarrow h h$ at leading order in the energy expansion [28].

We should finally point out the potential role of the rates for $h \rightarrow g g$ and $h \rightarrow \gamma \gamma$ in distinguishing a pseudo Nambu-Goldstone $h$ from a generic composite scalar. The basic remark [29] is that there are two classes of corrections to these rates. One correction originates from the modification of the coupling of $h$ to $W W$ and to $\bar{t} t$ and affects the on-shell $h \rightarrow g g, h \rightarrow \gamma \gamma$ amplitudes via the $W$ and $t$ loop contribution. In a sense this

\footnotetext{
${ }^{10}$ The effects from the tails of spin- 1 resonances can also be studied through the process $e^{+} e^{-} \rightarrow V V$, see for example refs. [117-122].
} 
contribution is long distance. A second correction is the genuine short-distance contribution to the Wilson coefficient of the operators

$$
\mathcal{O}_{g g}=h G_{\mu \nu} G^{\mu \nu}, \quad \mathcal{O}_{\gamma \gamma}=h F_{\mu \nu} F^{\mu \nu},
$$

that arises from loops of heavy states. In the case of a PNGB this second class of effects is suppressed with respect to the first by a factor $\left(g_{G^{*}} / g_{\rho}\right)^{2}$, where by $g_{G^{*}}$ we indicate a weak spurion coupling which breaks the Goldstone symmetry. This suppression is a consequence of the Goldstone symmetry selection rules and would be absent in the case of a generic composite scalar, like for instance the dilaton. In the limit where $g_{G} / g_{\rho} \ll 1$, the rates $h \rightarrow g g, h \rightarrow \gamma \gamma$ are fully controlled by $a$ and $c_{t}$ ( $c_{t}$ measures the deviations of the top Yukawa coupling [29]), a result that can in principle be tested. However, one should keep in mind that the measured value of $m_{h}$ prefers a scenario where the top partners are somewhat lighter than the rest and only moderately strongly coupled [108, 110, 127-129]. In that situation the correlation between $h \rightarrow g g, h \rightarrow \gamma \gamma$ and the parameters $a, c_{t}$ may receive important corrections.

\section{What can be learned from triple Higgs production?}

In this section we discuss the relevance of the process $V V \rightarrow h h h$ in distinguishing between a generic SILH and a PNGB (for an earlier study of this process at a linear collider, see ref. [130], while a study at the LHC has been recently carried out in ref. [131] and triple Higgs production by gluon fusion has also been studied in ref. [132]). We will show that this process is suppressed in the PNGB case as a consequence of a $Z_{2}$ invariance of the Lagrangian under which the NG bosons are odd. A priori, any three-body final state involving the Higgs and gauge bosons could be a further probe of the nature of the Higgs. In practice, however, $V V \rightarrow h h h$ is the only process that adds new information, thanks to its sensitivity to $b_{3}$. Studying further final states like $h h V, h V V$ and $V V V$ merely gives a complementary probe of the relation between $a^{2}$ and $b$.

\subsection{Symmetry structure}

In a symmetric coset like $\mathrm{SO}(5) / \mathrm{SO}(4)$ there exists a $Z_{2}$ invariance of the algebra (grading) under which the broken generators $T^{\hat{a}}$ change sign while the unbroken generators $T^{a}$ do not: $T^{a} \rightarrow+T^{a}, T^{\hat{a}} \rightarrow-T^{\hat{a}}$. At the field level this corresponds to a parity $R$ under which all NG bosons are odd

$$
R: \quad \pi^{\hat{a}}(x) \rightarrow-\pi^{\hat{a}}(x) .
$$

In general, $R$ is an accidental invariance of the Lagrangian at the two-derivative level and is violated at higher orders. This is for example the case of $\mathrm{SO}(4) / \mathrm{SO}(3)$, where $R$ coincides with the $P_{L R}$ parity of eq. (2.8). It may happen however, as for example in the case of $\mathrm{SO}(5) / \mathrm{SO}(4)$, that $R$ is an element of $\mathcal{G}$, in which case it remains unbroken to all orders in the derivative expansion of the strong dynamics. In fact, this is true for any coset $\mathcal{G} / \mathcal{H}$ 


\begin{tabular}{|c|c|c|}
\hline \multirow{2}{*}{ Polarisation } & \multicolumn{2}{|c|}{ Amplitude for } \\
& PNGB & SILH \\
\hline$V_{L} V_{L} \rightarrow h h h$ & $g^{2} v / f^{2}$ & $\hat{s} v / f^{4}$ \\
$V_{L} V_{T} \rightarrow h h h$ & $\sqrt{\hat{s}} g / f^{2}$ \\
$V_{T} V_{T} \rightarrow h h h$ & \multicolumn{2}{|c}{$g^{2} v / f^{2}$} \\
\hline
\end{tabular}

Table 1. Naive high-energy and large-angles behavior of partonic $V V \rightarrow h h h$ amplitudes for a PNGB Higgs (first column) and a generic SILH scalar (second column).

involving only doublets under some $\mathrm{SU}(2)^{\prime} \subset \mathcal{H} .{ }^{11}$ In particular this property is shared by the simplest cosets involving just one scalar doublet, whether custodially symmetric $(\mathrm{SO}(5) / \mathrm{SO}(4)$ or $\mathrm{SO}(4,1) / \mathrm{SO}(4))$ or not $(\mathrm{SU}(3) / \mathrm{SU}(2) \times \mathrm{U}(1))$. Consequently, when the Higgs doublet is the only PNGB multiplet from some strong dynamics, processes with an odd number of pseudo-NG bosons are forbidden to all orders in the strong dynamics and only arise as a weak effect of the SM couplings.

The above argument implies that, although by a naive counting one would expect the $V_{L} V_{L} \rightarrow h h h$ cross section to grow with $\hat{s}^{2},{ }^{12}$ this does not happen for a PNGB Higgs. In practice $R$ is weakly broken by the gauging, so that this process is not strictly zero but only suppressed by $g$. The expected energy behavior of the amplitude at the parton level can be estimated by power counting and is shown in table 1 . Longitudinal modes interact with coupling strength $\bar{g}(\sqrt{\hat{s}}) \sim \sqrt{\hat{s}} / f$, while transverse modes have weak coupling strength $g$. Measuring the cross section of triple Higgs production can thus give important indications on the nature of the Higgs boson and distinguish the case of a PNGB from that of a generic SILH. Indeed, as it will become more clear in a moment, the grading symmetry $R$ is reflected in some non-trivial correlations among the coefficients of operators of different dimensionality in the expansion of the effective lagrangian in powers of the Higgs doublet $H$.

\subsection{Quantitative analysis of $V V \rightarrow h h h$}

We checked that the expected cancellation of the energy-growing term of the $V_{L} V_{L} \rightarrow h h h$ scattering amplitude takes place by performing an explicit computation in the gaugeless limit $g=g^{\prime}=0$. By the equivalence theorem, the leading energy behavior of $V_{L} V_{L} \rightarrow h h h$ is captured by the NG boson scattering $\chi \chi \rightarrow h h h$. From the Lagrangian of eq. (2.1) we find three distinct diagrams, depicted in figure 2, plus their crossings, which contribute to the amplitude. At leading order in $\hat{s}$ we find ${ }^{13}$

$$
\mathcal{A}(\chi \chi \rightarrow h h h)=\frac{i \hat{s}}{v^{3}}\left(4 a b-4 a^{3}-3 b_{3}\right) .
$$

\footnotetext{
${ }^{11}$ Such a coset is obviously symmetric, as the commutator of any element in $\mathcal{G} / \mathcal{H}$ cannot be a doublet, and must therefore belong to $\mathcal{H}$. Moreover the residual $\mathrm{SU}(2)^{\prime}$ will forbid odd powers of the NG-bosons at any order in the derivative expansion.

${ }^{12}$ From here on we will indicate the partonic c.o.m. energy with $\hat{s}$, while $s$ will denote the collider energy.

${ }^{13}$ Note that, similarly to the process $V V \rightarrow h h$, anomalous Higgs self-interactions parametrized by $d_{3}$ and $d_{4}$ modify the amplitude of triple Higgs production near threshold but do not affect the asymptotic behavior at large partonic energy. Their contribution is thus subleading and will be neglected in the following.
} 

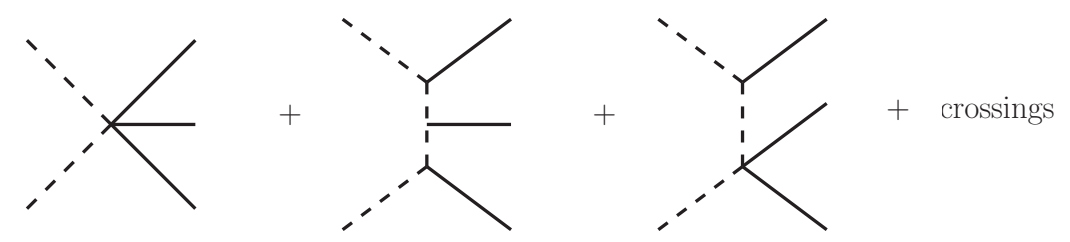

Figure 2. Leading diagrams contributing to the $\chi \chi \rightarrow h h h$ amplitude. Dashed lines represent the NG bosons $\chi$, while solid lines denote the Higgs boson $h$. The sum of these diagrams with their crossings cancels out exactly in the gaugeless limit for a symmetric coset and at the $O\left(p^{2}\right)$ level for any coset. See text.

\begin{tabular}{|c|ccccccc|}
\hline$\sigma$ & \multicolumn{7}{|c|}{$\xi$} \\
{$[\mathrm{ab}]$} & 0 & 0.05 & 0.1 & 0.2 & 0.3 & 0.5 & 0.99 \\
\hline PNGB & 0.32 & 0.46 & 0.71 & 1.47 & 2.41 & 4.13 & 0.30 \\
SILH & 0.32 & 0.71 & 0.87 & 7.56 & 42.89 & 407.9 & 7808 \\
\hline
\end{tabular}

Table 2. Cross section for the process $e^{+} e^{-} \rightarrow \nu \bar{\nu} h h h$ for $m_{h}=125 \mathrm{GeV}$ at $\sqrt{s}=3 \mathrm{TeV}$. The first line shows the cross sections obtained in the symmetric $\mathrm{SO}(5) / \mathrm{SO}(4)$ coset for various values of $\xi$. The cross sections in the second line are for a SILH with $c_{H}=1$ and $c_{H}^{\prime}=0$ and vanishing higher-order operators.

In the case of $\mathrm{SO}(5) / \mathrm{SO}(4)$ the values of the couplings $a, b$ and $b_{3}$ are given by eq. (2.13) and the coefficient of the term growing with $\hat{s}$ in the amplitude vanishes identically. In the case of a generic Higgs doublet the cancellation works at the $O\left(v^{2} / f^{2}\right)$ level, as due to the universality of the SILH Lagrangian, but it fails at higher orders. By substituting the relations of eq. (2.11) into eq. (5.2) we find

$$
\mathcal{A}(\chi \chi \rightarrow h h h)=2 i\left(c_{H}^{\prime}-2 c_{H}^{2}\right) \frac{\hat{s}}{v^{3}}\left(\frac{v^{4}}{f^{4}}\right) .
$$

As expected, the coefficient of the energy-growing term is of order $v^{4} / f^{4}$ and proportional to the linear combination $\left(c_{H}^{\prime}-2 c_{H}^{2}\right)$. This latter must vanish if the Higgs lives on a symmetric $\operatorname{coset} \mathcal{G} / \mathcal{H} .^{14}$

At CLIC, triple Higgs production proceeds through the process $e^{+} e^{-} \rightarrow \nu \bar{\nu} V V \rightarrow$ $\nu \bar{\nu} h h h$, where $V=W^{ \pm}, Z$. Some typical values of the cross section are shown in table 2 for the case of a PNGB and a SILH with $c_{H}=1$ and $c_{H}^{\prime}=0$ (and vanishing higher-order operators). While the cross section for a PNGB is in the range of a few ab, in the case of a generic SILH it can be much bigger and grows like $\xi^{4}$, with the dominant contribution coming from the subprocess $V_{L} V_{L} \rightarrow h h h$. A careful analysis of the sensitivity of a linear collider to the anomalous couplings involved in triple Higgs production is beyond the scope of this work. A very conservative approach is to decay every Higgs to $b \bar{b}$ and to require the identification of at least $5 b$-jets. The branching ratio of three Higgses into $3 b \bar{b}$ pairs is $20 \%$. Assuming an $80 \% b$-tagging probability, the efficiency to reconstruct at least $5 b$-jets out of the available 6 in the final state is $66 \%$. Including an additional factor 3 reduction

\footnotetext{
${ }^{14}$ This shows that the relation $c_{H}^{\prime}=2 c_{H}^{2}$ holds true in any symmetric coset, and not only in $\mathrm{SO}(5) / \mathrm{SO}(4)$.
} 


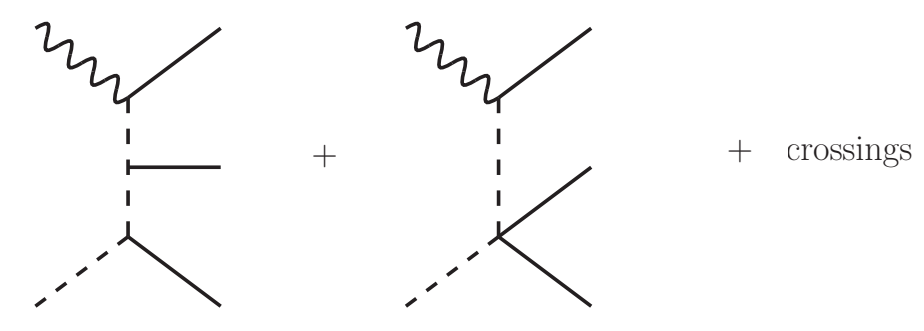

Figure 3. Diagrams giving the dominant contribution to the $V_{T} \chi \rightarrow h h h$ cross-section. Continuous, dashed and wiggly lines denote a Higgs boson $h$, the NG bosons $\chi$, and a transverse gauge boson $V_{T}$ respectively.

due to identification cuts to be performed on the final state jets one obtains an overall efficiency on the signal which is roughly $5 \%$. Requiring the identification of $O(10)$ triple Higgs events implies the possibility to detect this process with an integrated luminosity of $1 \mathrm{ab}^{-1}$ as soon as $\xi \gtrsim 0.3$ for a generic SILH.

The dominant contribution to triple Higgs production in the case of a PNGB Higgs comes from the subprocess $W_{L}^{ \pm} W_{T}^{\mp} \rightarrow h h h$, whose cross section is expected to grow as $\hat{s} \log \hat{s}$. The leading logarithmic behavior can be extracted by using the equivalence theorem and arises from the subset of diagrams shown in figure 3. In the limit in which the intermediate PNGB line is nearly on-shell, the total cross section factorizes into the product of a collinear $W_{T} \rightarrow \chi h$ splitting times the cross section of a hard $\chi^{ \pm} \chi^{\mp} \rightarrow h h$ scattering

$$
\sigma\left(W_{T}^{ \pm} \chi^{\mp} \rightarrow h h h\right)=\int d x d p_{T}^{2} f\left(x, p_{T}\right) \sigma\left(\chi^{ \pm} \chi^{\mp} \rightarrow h h\right)(x \hat{s}) .
$$

Here $x$ is the fraction of the $W$ energy carried by the emitted $\chi, p_{T}$ is its transverse momentum and $\hat{s}$ is the total center-of-mass energy of the $W_{T} \chi \rightarrow h h h$ process. The splitting function $f\left(x, p_{T}\right)$ can be calculated using eq. (2.1) and is given by

$$
f\left(x, p_{T}\right)=\frac{1}{p_{T}^{4}} \frac{x(1-x)}{8 \pi^{2}}\left|\mathcal{A}\left(W_{T} \rightarrow \chi h\right)\right|^{2}=\frac{x(1-x)}{p_{T}^{2}} \frac{a^{2} g^{2}}{32 \pi^{2}},
$$

where we have neglected the masses of the gauge and Higgs bosons. Notice that the amplitude of the splitting $\mathcal{A}\left(W_{T} \rightarrow \chi h\right)$ vanishes in the forward direction as required by angular momentum conservation. At leading order in $\hat{s}$, the cross section of the hard $\chi \chi \rightarrow h h$ scattering does not depend on $p_{T}$, and reads

$$
\sigma\left(\chi^{ \pm} \chi^{\mp} \rightarrow h h\right)(\hat{s})=\frac{\hat{s}\left(b-a^{2}\right)^{2}}{32 \pi v^{4}} .
$$

We thus obtain

$$
\sigma\left(W_{T}^{ \pm} \chi^{\mp} \rightarrow h h h\right)=\frac{g^{2}}{12288 \pi^{3}} \frac{\left(a b-a^{3}\right)^{2}}{v^{4}} \hat{s} \log \frac{\hat{s}}{m_{W}^{2}} .
$$

The factor $\log \left(\hat{s} / m_{W}^{2}\right)$ originates from the logarithmic divergence of the integral over $p_{T}$, which is cut off in the infrared at $p_{T}^{2} \sim m_{W}^{2}$ once the $W$ mass dependence is properly taken into account. 


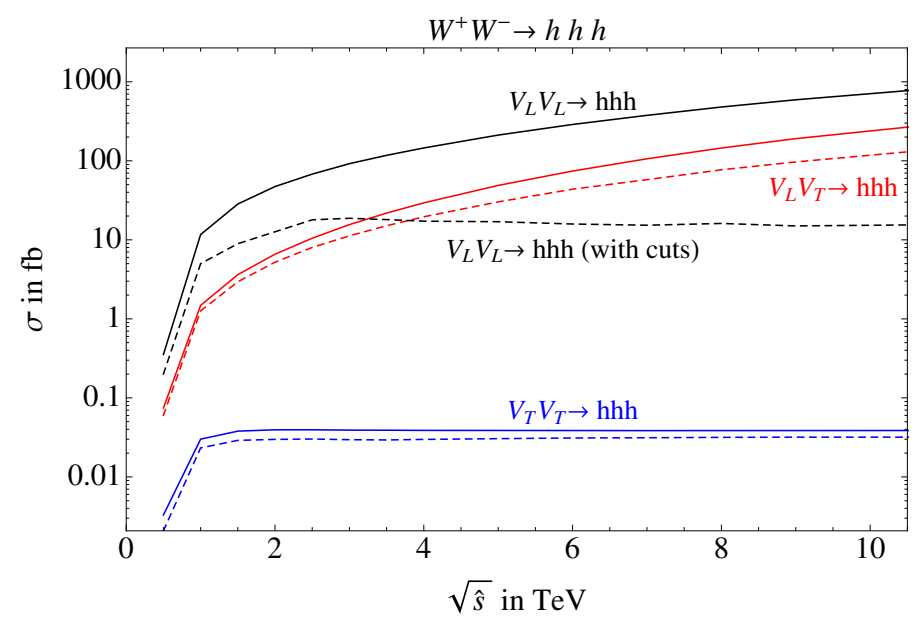

Figure 4. Partonic cross-sections of the processes $V_{L} V_{L} \rightarrow h h h$ (black), $V_{L} V_{T} \rightarrow h h h$ (red) and $V_{T} V_{T} \rightarrow h h h$ (blue) as a function of $\sqrt{\hat{s}}$ for a PNGB Higgs with $\xi=0.1$ and $m_{h}=125 \mathrm{GeV}$. The dashed line shows the partonic cross section after applying the cuts $p_{T}>0.05 \sqrt{\hat{s}}$ for each Higgs and $m_{h h}>0.1 \sqrt{\hat{s}}$ for all Higgs pairs.

Although this calculation captures the exact asymptotic behavior of the $W_{T}^{ \pm} W_{L}^{\mp} \rightarrow$ hhh process, it turns out that the subleading contribution proportional to $\hat{s}$ is numerically large, so that the logarithmically enhanced term starts to dominate only at very high center-of-mass energies. The energy dependence of the process $V V \rightarrow h h h(V=W, Z)$ in the various polarization channels is shown in figure 4. We assume that the Higgs boson is a PNGB living on the coset $\mathrm{SO}(5) / \mathrm{SO}(4)$, so that the selection rules discussed at the beginning of this section apply. The expectation for the various $2 \rightarrow 3$ amplitudes is reported in table 1 . Notice that for a three-body process the product of the flux factor and phase space is dimensionless. The naive high-energy behavior of the various cross sections is then obtained by squaring the entries of table 1 . The total cross section is expected to follow this naive energy behavior only if the phase space integral does not get any particular enhancement from singular kinematic configurations. As explained above, the $L T$ polarization channel indeed gets a logarithmic enhancement from the kinematical region where one of the final Higgs bosons is collinear with the incoming transverse vector. As figure 4 shows, the $T T$ channel cross section is constant at high energy, in agreement with the naive expectation $\sigma\left(V_{T} V_{T} \rightarrow h h h\right) \sim\left(g^{4} /(4 \pi)^{3}\right)\left(v^{2} / f^{4}\right)$. On the other hand the inclusive $V_{L} V_{L} \rightarrow h h h$ cross section grows like $\hat{s}$ at high energy, faster than what is expected from table 1. This is due to the Coulomb singularity that some of the diagrams have in the limit in which one of the final Higgs bosons is collinear to the incoming beam. The amplitude of those diagrams goes as $\mathcal{A}\left(V_{L} V_{L} \rightarrow h h h\right) \sim\left(g^{2} v / f^{2}\right)(\hat{s} / \hat{t})$, where $\hat{t}$ is the squared difference of one of the initial momenta and the momentum of the collinear Higgs boson. The integral over $\hat{t}$ is dominated by the singular region $t \simeq t_{\min } \sim-m_{W}^{2}$, so that the total cross section gets enhanced by a factor $\left(\hat{s} / m_{W}^{2}\right)$. Such an enhancement can be removed by suitable kinematic cuts to avoid all collinear configurations. For example, the dashed curves of figure 4 show the energy dependence of the cross sections after requiring $p_{T}>0.05 \sqrt{\hat{s}}$ on each of the Higgs final momenta. As expected, the TT and $L T$ channels 
are only slightly suppressed by the cuts, while the Coulomb singularity of the $L L$ channel is removed and its cross section is constant at high energies.

\section{Quantitative analysis of $V V \rightarrow V V$ and $V V \rightarrow h h$}

In this section we study the sensitivity of a linear $e^{+} e^{-}$collider to the anomalous Higgs couplings $a, b$ and $d_{3}$ through vector boson scattering and double Higgs production. In section 6.2 we discuss $V_{L} V_{L} \rightarrow V_{L} V_{L}$ scattering at low-energy (ILC) and high-energy (CLIC) linear colliders; in sections 6.3 and 6.4 we focus on double Higgs production. We show that, while CLIC can provide a precise determination of the anomalous couplings through the study of the vector boson fusion process $e^{+} e^{-} \rightarrow h h \nu \bar{\nu}$, a lower-energy machine like the ILC has to rely on the double Higgs-strahlung process $e^{+} e^{-} \rightarrow h h Z$. Previous analyses of $V V \rightarrow V V$ and $V V \rightarrow h h$ at high-energy linear colliders appeared in refs. [133-135] and $[17,137,138]$ respectively. ${ }^{15}$

\subsection{Identification cuts}

In the following we set $m_{h}=125 \mathrm{GeV}$ and focus on final states where $W, Z$ and $h$ decay hadronically, with the only exception of double Higgs-strahlung where we include leptonic decays of the $Z$. Our analysis is at the parton level and does not include corrections due to QCD radiation. Final-state partons are passed through a simple algorithm to obtain a crude though sufficiently accurate approximation of jet-reconstruction and detector effects of a real experiment. We perform a simple Gaussian smearing of the parton energies assuming a constant resolution $\Delta E / E=5 \%$ [14-16]. Two partons with

$$
\Delta R_{j j}<0.4
$$

are merged together by summing their 4-momenta. The algorithm is applied recursively until it converges to a list of final partons. A parton thus obtained which satisfies the cuts

$$
E_{j}>20 \mathrm{GeV}, \quad\left|\eta_{j}\right|<2
$$

is identified with a reconstructed jet. The rapidity cut, in particular, excludes reconstructed jets which fall within $15^{\circ}$ of the collision axis. Leptons (muons and electrons) are identified if they satisfy the following cuts:

$$
E_{\ell}>5 \mathrm{GeV}, \quad\left|\eta_{\ell}\right|<2 .
$$

We furthermore require a separation $\Delta R_{j \ell}>0.4$ between reconstructed jets and leptons.

In events with two Higgs bosons (like those coming from $W W$ fusion or double Higgsstrahlung) we require at least $3 b$-tags with a $b$-tagging efficiency of $80 \%$ assumed throughout the analysis. According to ref. [14-16], this is associated with mistag rates of $\sim 10 \%$ and $\sim 1 \%$ for $c$-jets and light jets respectively. With this assumption the probability of tagging at least $3 b$-jets out of 4 is $82 \%$.

\footnotetext{
${ }^{15}$ See also ref. [136] for a study of the process $\gamma \gamma \rightarrow V V(V=W, Z)$ to extract the anomalous Higgs couplings.
} 
All our event samples are generated with MADGraph5 [139], except for the background to $V V \rightarrow V V$ scattering which has been generated with Whizard [140]. We do not include parton showering and hadronization.

\section{2 $V V \rightarrow V V$ scattering}

All the channels $e^{+} e^{-} \rightarrow V V \ell \bar{\ell}$, where $\ell$ is either an electron or a neutrino, provide a framework for studying vector boson scattering at a linear collider. $V V$ scattering processes with electrons in the final state are initiated by a neutral current splitting $e^{ \pm} \rightarrow \gamma e^{ \pm}$or $e^{ \pm} \rightarrow Z e^{ \pm}$. While the first always contributes as a large background to the signal we are interested in, the second splitting is a factor of 2 smaller than the charged current splitting $e \rightarrow W \nu$. We will focus therefore on $V V \nu \bar{\nu}$ final states and neglect $V V e^{+} e^{-}$for simplicity. We will consider hadronically decaying vector bosons and, to avoid possible experimental issues related to energy resolution and $W / Z$ separation, we will be inclusive and sum over final states with $W$ 's or $Z$ 's.

The cross section for the process $e^{+} e^{-} \rightarrow V V \nu \bar{\nu}$ can be parametrized in terms of the coupling shift $\Delta a^{2}$ as

$$
\sigma\left(\Delta a^{2}\right)=\sigma_{S M}\left(1+A \Delta a^{2}+B\left(\Delta a^{2}\right)^{2}\right)
$$

where $\sigma_{S M}$ is the SM cross section and $A, B$ are two dimensionless coefficients. Notice that $\Delta a^{2}=\xi$ in the MCHM. For $\sqrt{s}=3 \mathrm{TeV}$ we find, before any cut,

$$
\sigma_{S M}=184 \mathrm{fb}, \quad\{A, B\}=\{0.01,0.15\} \quad\left[\begin{array}{cc}
e^{+} e^{-} \rightarrow V V \nu \bar{\nu} & \sqrt{s}=3 \mathrm{TeV} \\
\text { before any cut }
\end{array}\right] .
$$

It is clear that at this level the cross section is largely dominated by the SM term. One reason is the "accidental" numerical enhancement, discussed in ref. [28], of the partonic $V_{T} V_{T} \rightarrow V_{T} V_{T}$ cross section compared to $V_{L} V_{L} \rightarrow V_{L} V_{L}$. Another reason is that the total cross section displayed here is dominated by threshold production and does not really probe the highest energies.

The situation is worsened by the presence of backgrounds. The largest contribution arises from the process $e^{+} e^{-} \rightarrow W^{+} W^{-} e^{+} e^{-}$, which goes through a $\gamma \gamma \rightarrow W^{+} W^{-}$hard scattering, where the final electron and positron escape the detector. A similar though smaller background comes from $e^{+} e^{-} \rightarrow W^{ \pm} Z e^{\mp} \nu$. Before cuts, the cross section of these background processes is of the order of hundreds of picobarns.

We focus on hadronic decays of the $W$ and $Z$ bosons and select events with at least four reconstructed jets, where jet reconstruction is done according to the procedure discussed in section 6.1. The two $V$ candidates are defined by considering the four most energetic jets in each event, $j_{1, \ldots 4}$, and by identifying the pairing $\left(j_{1} j_{2}, j_{3} j_{4}\right)$ which minimizes the $\chi^{2}$ function

$$
\left(m_{j_{1} j_{2}}-m_{V}\right)^{2}+\left(m_{j_{3} j_{4}}-m_{V}\right)^{2},
$$

where $m_{V} \equiv\left(m_{W}+m_{Z}\right) / 2$. We use the average mass $m_{V}$ in the $\chi^{2}$ function since we do not know a priori if the $V$ candidate is a $W$ or a $Z$. The algorithm is however quite effective to identify real vector bosons, and the percentage of fake pairings is negligible. 
After their reconstruction, we impose the following cut on the invariant mass of each of the two $V$ candidates:

$$
\left|m_{j j}-m_{V}\right|<15 \mathrm{GeV}
$$

Events where such requirement is not satisfied are discarded. The overall efficiency of the identification cuts in eqs. (6.2) and (6.7) is roughly $30 \%$ for both signal and background. After imposing the identification cuts and including the hadronic branching ratios of the $W$ and $Z$ bosons, we find that the signal rate $r=\sigma\left(e^{+} e^{-} \rightarrow V V \nu \bar{\nu}\right) \times B R(V V \rightarrow 4 j)$ is parametrized by

$$
r\left(\Delta a^{2}\right)=r_{S M}\left(1+A_{r} \Delta a^{2}+B_{r}\left(\Delta a^{2}\right)^{2}\right)
$$

with

$$
r_{S M}=28.7 \mathrm{fb}, \quad\left\{A_{r}, B_{r}\right\}=\{0.04,0.16\} \quad\left[\begin{array}{cc}
e^{+} e^{-} \rightarrow 4 j \nu \bar{\nu} & \sqrt{s}=3 \mathrm{TeV} \\
\text { after identification cuts }
\end{array}\right] .
$$

In order to enhance the signal and reduce the backgrounds we apply the following additional set of cuts

$$
\begin{aligned}
m_{V_{1} V_{2}} & >500 \mathrm{GeV}, \\
\min p_{T}\left(V_{i}\right) & >100 \mathrm{GeV}, \\
\max \left|\eta_{V_{i}}\right| & <1.1, \\
m_{\nu \nu} & >150 \mathrm{GeV},
\end{aligned}
$$

where $V_{1,2}$ denote the two $V$ candidates. The cut on the invariant mass of the two neutrinos, in particular, eliminates those backgrounds, like $e^{+} e^{-} \rightarrow V V Z$ (with $Z \rightarrow \nu \bar{\nu}$ ), where the missing energy arises from the invisible decay of an on-shell $Z$ boson. Finally, we require

$$
p_{T}\left(V_{1} V_{2}\right)>75 \mathrm{GeV}
$$

This latter cut on the transverse momentum of the $V V$ system is applied to further reduce the $e^{+} e^{-} \rightarrow W^{+} W^{-} e^{+} e^{-}$and $e^{+} e^{-} \rightarrow W^{ \pm} Z e^{\mp} \nu$ backgrounds: if the final electrons are so forward to be lost in the beam-pipe it is reasonable to expect the total $p_{T}$ of the recoiling vectors to be small. After all these cuts, the signal rate is parametrized by

$$
r_{S M}=1.7 \mathrm{fb}, \quad\left\{A_{r}, B_{r}\right\}=\{0.04,0.7\} \quad\left[\begin{array}{cc}
e^{+} e^{-} \rightarrow 4 j \nu \bar{\nu} & \sqrt{s}=3 \mathrm{TeV} \\
\text { after analysis cuts }
\end{array}\right] .
$$

The background rate from $e^{+} e^{-} \rightarrow W^{+} W^{-} e^{+} e^{-}$and $e^{+} e^{-} \rightarrow W^{ \pm} Z e^{\mp} \nu$ processes amounts to roughly $r_{b}=2.5 \mathrm{fb}$ after the cuts. The calculation has been performed using WhIZARD [140] by requiring the electrons in the final state to be undetected $\left(\eta\left(e^{ \pm}\right)>2.5\right) \cdot{ }^{16}$

\footnotetext{
${ }^{16}$ We found significant numerical instabilities in the $\mathrm{MC}$ computation of the cross section, with variations in the final result up to $30-50 \%$. As explained below, we took into account such uncertainty in our analysis by rescaling the final background rate by a factor 1.5 .
} 


\begin{tabular}{|c|cc|}
\hline$\Delta \bar{a}^{2}=\bar{\xi}$ & $\Delta a^{2}$ & $\xi$ \\
\hline 0 & $(-0.21,0.17)$ & $(0,0.17)$ \\
0.05 & $(-0.22,0.17)$ & $(0,0.18)$ \\
0.1 & $(-0.23,0.18)$ & $(0,0.19)$ \\
0.2 & $(-0.34,-0.1) \cup(0.04,0.28)$ & $(0.06,0.28)$ \\
0.3 & $(-0.45,-0.22) \cup(0.17,0.39)$ & $(0.17,0.39)$ \\
0.5 & $(-0.62,-0.49) \cup(0.45,0.56)$ & $(0.43,0.56)$ \\
\hline
\end{tabular}

Table 3. Expected $68 \%$ probability intervals on $\Delta a^{2}$ (second column) and $\xi$ (third column) for different true values $\Delta \bar{a}^{2}=\bar{\xi}$ measured at CLIC $3 \mathrm{TeV}$ through $V V \rightarrow V V$ scattering. The limits on $\xi$ have been derived by taking into account that only values in the range $\xi \in[0,1]$ are theoretically allowed. See the text for details on the statistical analysis.

We thus proceed to estimate the expected sensitivity on $\Delta a^{2}$. We follow a Bayesian approach and construct a posterior probability for the total event rate $r_{\text {tot }}$

$$
p\left(r_{\text {tot }} \mid N_{\text {obs }}\right) \propto \mathcal{L}\left(N_{\text {obs }} \mid r_{\text {tot }} L\right) \pi\left(r_{\text {tot }}\right),
$$

where $N_{\text {obs }}$ is the assumed number of observed events and $L$ is the integrated luminosity. We denote with $\pi\left(r_{\text {tot }}\right)$ the prior distribution and with $\mathcal{L}\left(N_{\text {obs }} \mid r_{\text {tot }} L\right)$ the likelihood function, which we take to be a Poisson distribution

$$
\mathcal{L}\left(N_{\text {obs }} \mid r_{\text {tot }} L\right)=\frac{e^{-r_{\text {tot }} L}\left(r_{\text {tot }} L\right)^{N_{\text {obs }}}}{N_{\text {obs }} !} .
$$

For a given true value $\Delta \bar{a}^{2}$ of the coupling shift, we assume the number of observed events to be $N_{\text {obs }}=\left(r\left(\Delta \bar{a}^{2}\right)+r_{b}\right) L$, while the total rate is $r_{\text {tot }}=r\left(\Delta a^{2}\right)+r_{b}$. As we do not explicitly introduce additional uncertainties (theoretical or systematic) on the estimate of the background in our statistical analysis ${ }^{17}$ we have conservatively rescaled the background rate $r_{b}$ by a factor 1.5 compared to the MC prediction.

By assuming a flat prior on $\Delta a^{2}$ and setting the integrated luminosity to $L=1 \mathrm{ab}^{-1}$, we obtain the $68 \%$ probability intervals shown in table 3 (second column) for different true values $\Delta \bar{a}^{2}$. We find that for large $\Delta \bar{a}^{2}$, the term proportional to $\left(\Delta a^{2}\right)^{2}$ dominates the rate and a second peak of the likelihood appears at negative values of the coupling shift. The $68 \%$ interval in these cases consists of two disconnected parts. We also considered the $\mathrm{SO}(5) / \mathrm{SO}(4)$ models MCHM where the coupling shift is $\Delta a^{2}=\xi$, see eq. (2.13). In this case we have imposed a prior on $\xi$ which is flat in the theoretically allowed range $[0,1]$ and vanishing outside. The corresponding $68 \%$ probability intervals on $\xi$ are reported in the third column of table 3 for different true values $\bar{\xi}$. With our set of cuts, a $3 \mathrm{TeV}$

\footnotetext{
${ }^{17}$ This would require introducing one or more corresponding nuisance parameters in the likelihood function, which is beyond the scope of our simple statistical analysis.
} 
linear collider is sensitive to values of $\Delta a^{2}(\xi)$ bigger than $\sim 0.2$ through $W W$ scattering. ${ }^{18}$ We do not find any significant gain in resolution by applying a harder cut on the $V V$ invariant mass.

A similar analysis can be carried out for a lower energy machine. We considered for example the case of a $500 \mathrm{GeV}$ linear collider. Parametrizing the signal cross section as in eq. (6.4), before cuts we find

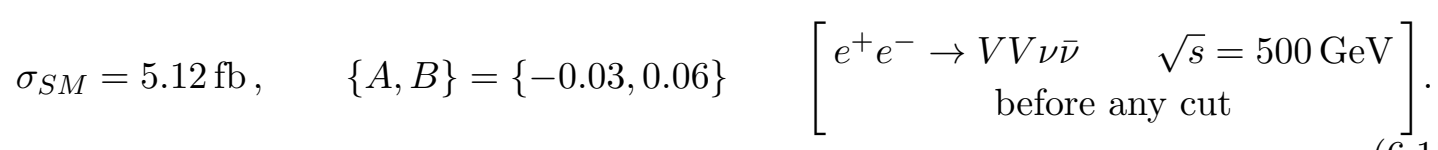

At this stage the background processes have very large cross sections, of the order of $100 \mathrm{fb}$. However, all backgrounds can be reduced to a negligible level by applying the identification cuts of eqs. (6.2) and (6.7), the additional cuts $p_{T}\left(V_{1} V_{2}\right)>40 \mathrm{GeV}, m_{\nu \nu}>100 \mathrm{GeV}$ and requiring all electrons in the final states to escape detection, $\eta\left(e^{ \pm}\right)>2.4$. After these cuts, and including the hadronic branching ratio of $W$ and $Z$, the signal rate is parametrized as in eq. (6.8) with:

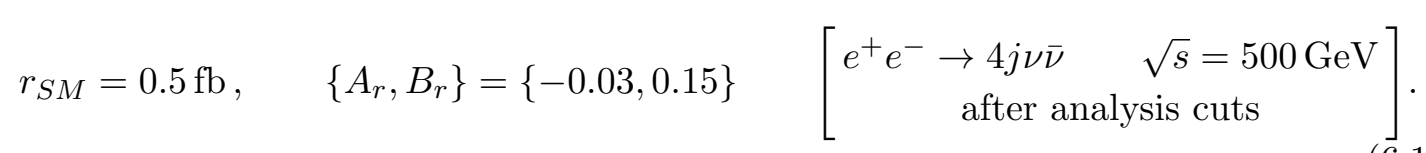

By repeating the previous statistical analysis, we find that with an integrated luminosity $L=1 \mathrm{ab}^{-1}$ the effect of a non-vanishing $h V V$ anomalous coupling can be resolved in $e^{+} e^{-} \rightarrow V V \nu \bar{\nu}$ only for large values of $\Delta a^{2}(\xi)$, of the order $0.5-0.6$.

\section{3 $V V \rightarrow h h$ scattering}

The scattering amplitude for $V_{L} V_{L} \rightarrow h h$ depends on $a, b$ and $d_{3}$ and can be conveniently written as $\mathcal{A}=a^{2}\left(\mathcal{A}_{S M}+\mathcal{A}_{1} \delta_{b}+\mathcal{A}_{2} \delta_{d_{3}}\right)$, where $\mathcal{A}_{S M}$ is the value predicted by the SM and ${ }^{19}$

$$
\delta_{b} \equiv 1-\frac{b}{a^{2}}, \quad \delta_{d_{3}} \equiv 1-\frac{d_{3}}{a} .
$$

At large partonic center-of-mass energies, $E \gg m_{V}, \mathcal{A}_{1}$ grows like $E^{2}$, while $\mathcal{A}_{2}$ and $\mathcal{A}_{S M}$ are constant. The parameter $\delta_{b}$ thus controls the high-energy behavior of the amplitude and gives a genuine "strong coupling" signature. On the contrary, $\delta_{d_{3}}$ determines the value of the cross section at threshold [28]. In an $e^{+} e^{-}$collider, $V_{L} V_{L} \rightarrow h h$ scatterings can be studied via the processes $e^{+} e^{-} \rightarrow \nu \bar{\nu} h h$ and $e^{+} e^{-} \rightarrow e^{+} e^{-} h h$. The latter, initiated by a partonic $Z Z$ state, has a cross section which is roughly one order of magnitude smaller than the former. This is due in particular to the fact that the $e^{ \pm} \rightarrow Z e^{ \pm}$splitting function is roughly a factor of 2 smaller than $e^{ \pm} \rightarrow W^{ \pm} \nu$. For this reason we neglect $e^{+} e^{-} \rightarrow e^{+} e^{-} h h$ in the following. The $e^{+} e^{-} \rightarrow \nu \bar{\nu} h h$ cross section can be written as

$$
\sigma=a^{4} \sigma_{S M}\left(1+A \delta_{b}+B \delta_{d_{3}}+C \delta_{b} \delta_{d_{3}}+D \delta_{b}^{2}+E \delta_{d_{3}}^{2}\right)
$$

\footnotetext{
${ }^{18}$ Here and in the following, by sensitivity/precision on some anomalous Higgs coupling we mean the $68 \%$ error on its measured value for injected SM signal.

${ }^{19}$ In the MCHM4 $\delta_{b}=\xi /(1-\xi), \delta_{d_{3}}=0$, while in the MCHM5 $\delta_{b}=\delta_{d_{3}}=\xi /(1-\xi)$. See eqs. $(2.13),(2.14)$
} 
where $\sigma_{S M}$ denotes its SM value. Notice that $a$ enters only as an overall factor. Without applying any kinematic cut on the Higgs decay products (nor including the branching fraction of Higgs decays) we find, for $\sqrt{s}=3 \mathrm{TeV}$,

$$
\sigma_{S M}=0.83 \mathrm{fb}, \quad\{A, B, C, D, E\}=\{3.83,0.64,3.41,15.6,0.48\} \quad\left[\begin{array}{c}
\sqrt{s}=3 \mathrm{TeV} \\
\text { before any cut }
\end{array}\right] .
$$

Notice that although the SM cross section $\sigma_{S M}$ of the processes $V V \rightarrow V V$ and $V V \rightarrow h h$ differs by more than two orders of magnitude, the energy-growing contributions (given by $\sigma_{S M} B=27.6 \mathrm{fb}$ for $V V \rightarrow V V$, see eqs. (6.4) and (6.5), and $\sigma_{S M} D=12.9 \mathrm{fb}$ for $V V \rightarrow h h$, see eqs. (6.18) and (6.19)) are of the same size, as required by the $\mathrm{SO}(4)$ invariance.

In contrast to $V V \rightarrow V V$ scattering, in the case of double Higgs production simple acceptance and reconstruction cuts keep the background at a negligible level. For our analysis we focus on events where both Higgs bosons in the signal decay to $b \bar{b}$, and select events with four or more jets and at least three $b$-tags. The most important processes which can fake the signal are then $e^{+} e^{-} \rightarrow \nu \bar{\nu} h Z, e^{+} e^{-} \rightarrow \nu \bar{\nu} Z Z$ and $e^{+} e^{-} \rightarrow e^{+} e^{-} Z Z$. In all cases the $Z$ boson must decay to a $b \bar{b}$ pair, and in the latter process both electrons have to be missed in the beam pipe. Before cuts we find

$$
\begin{aligned}
\sigma\left(e^{+} e^{-} \rightarrow h Z \nu \bar{\nu} \rightarrow b \bar{b} b \bar{b} \nu \bar{\nu}\right) & =0.88 \mathrm{fb}, \\
\sigma\left(e^{+} e^{-} \rightarrow Z Z \nu \bar{\nu} \rightarrow b \bar{b} b \bar{b} \nu \bar{\nu}\right) & =1.26 \mathrm{fb}, \\
\sigma\left(e^{+} e^{-} \rightarrow Z Z e^{+} e^{-} \rightarrow b \bar{b} b \bar{b} e^{+} e^{-}\right) & =0.58 \mathrm{fb},
\end{aligned}
$$

which can be compared to the signal cross section in eq. (6.19) after multiplying this latter by the Higgs pair branching fraction $B R(h h \rightarrow b \bar{b} b \bar{b}) \simeq B R(h h \rightarrow b \bar{b} b \bar{b})_{S M}=0.34$. Further backgrounds, like for example $t \bar{t} \rightarrow b \bar{b} W^{+} W^{-} \rightarrow b \bar{b} j j l \nu$, can fake our signal only if one or more light jets are mistagged as $b$-jets and if extra charged leptons escape into the beam pipe. This is enough suppression to safely ignore them. The backgrounds in eq. (6.20), on the other hand, are largely suppressed, and thus negligible, if the jet energy resolution of the detector is sufficiently good to accurately distinguish a $Z$ from a Higgs boson. This seems to be a valid assumption according to ref. [14-16], and in the following we will consequently assume the backgrounds to be negligible.

A simple-minded approach to the extraction of the two parameters $\delta_{b}$ and $\delta_{d_{3}}$ is the following. Let us consider a kinematical variable $\mathcal{O}$ whose value increases with the c.o.m. energy of the $W^{+} W^{-} \rightarrow h h$ subprocess. The invariant mass of the two Higgses, $m_{h h}$, and the sum of their transverse momenta, $H_{T}$, are two valid examples for $\mathcal{O}$. We can divide the set of $e^{+} e^{-} \rightarrow \nu \bar{\nu} h h$ events into two categories according to whether $\mathcal{O}<\overline{\mathcal{O}}$ or $\mathcal{O}>\overline{\mathcal{O}}$, where $\overline{\mathcal{O}}$ is some fixed value. The number of observed events in these two categories can be fitted to $\sigma_{<}\left(\delta_{b}, \delta_{d_{3}}\right)$ and $\sigma_{>}\left(\delta_{b}, \delta_{d_{3}}\right)$. Notice that thanks to the cut on $\mathcal{O}, \sigma_{>}\left(\delta_{b}, \delta_{d_{3}}\right)$ will have an enhanced sensitivity to $\delta_{b}$ while $\sigma_{<}\left(\delta_{b}, \delta_{d_{3}}\right)$ is more sensitive to $\delta_{d_{3}}$.

We thus adopt the above strategy and proceed as follows. We start by selecting events with four or more reconstructed jets. The Higgs candidates are identified from the list of the four most energetic jets, $j_{1, \ldots 4}$, by selecting the pairing $\left(j_{1} j_{2}, j_{3} j_{4}\right)$ which minimizes the 

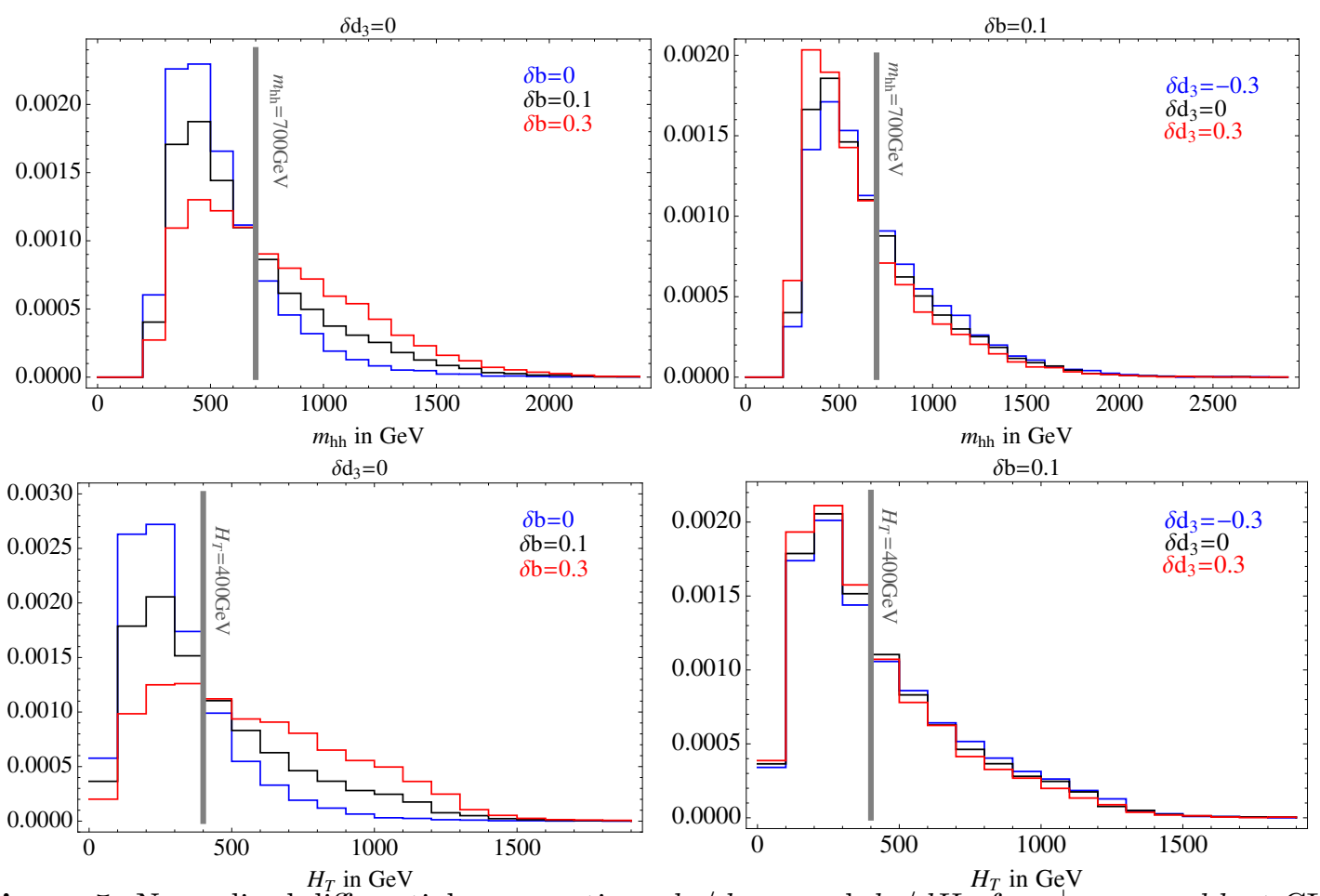

Figure 5. Normalized differential cross sections $d \sigma / d m_{h h}$ and $d \sigma / d H_{T}$ for $e^{+} e^{-} \rightarrow \nu \bar{\nu} h h$ at CLIC with $\sqrt{s}=3 \mathrm{TeV}$ after the identification cuts of eqs. (6.2) and (6.22), for several values of $\delta_{b}$ and $\delta_{d_{3}}$.

$\chi^{2}$ function

$$
\left(m_{j_{1} j_{2}}-m_{h}\right)^{2}+\left(m_{j_{3} j_{4}}-m_{h}\right)^{2} .
$$

We impose the following cut on the invariant mass of each of the two Higgs candidates

$$
\left|m_{j j}-m_{h}\right|<15 \mathrm{GeV},
$$

and require that at least three of the jets $j_{1, \ldots 4}$ are $b$-tagged. Events where these requirements are not fulfilled are discarded. We find that the overall efficiency of the identification cuts of eqs. (6.1), (6.2) and (6.22) varies from $20 \%$ to roughly $35 \%$ when $\delta_{b}$ ranges in the interval $0-0.5$, while it is only marginally sensitive to $\delta_{d_{3}}$. In particular, the energy cut on the jets has an almost constant efficiency (roughly $80 \%$ ) over the whole parameter space. The variation in the total efficiency comes mainly from the cuts on pseudorapidity and on $\Delta R$. The cut on $\eta$ disfavors small values of $\delta_{b}$, since these typically lead to more forward Higgses and consequently more forward $b$-jets, which in turn have a smaller probability to pass the $\eta$ cut. The cut on minimum $\Delta R$, eq. (6.1), on the other hand, disfavors large values of $\delta_{b}$, since these lead to more boosted Higgses and thus more collimated decay products. Finally, the cut in eq. (6.22) has an almost unit efficiency in our parton-level analysis with our assumed energy resolution.

Figure 5 shows the distributions of $m_{h h}$ and $H_{T}$ for some fixed values of the parameters $\delta_{b}$ and $\delta_{d_{3}}$ after the identification cuts. While a single cut on either of these two kinematic variables is sufficient to extract the dependence on $\delta_{b}$ and $\delta_{d_{3}}$, we found that 


\begin{tabular}{|c|cccccc|}
\hline & $r_{S M}[\mathrm{ab}]$ & $A_{r}$ & $B_{r}$ & $C_{r}$ & $D_{r}$ & $E_{r}$ \\
\hline I & 8.8 & 15.6 & 0.88 & 14.5 & 164 & 0.07 \\
II & 4.5 & 3.87 & 0.30 & 0.92 & 4.44 & -0.08 \\
III & 6.5 & 9.89 & 1.25 & 17.1 & 55.4 & 1.54 \\
IV & 44 & 3.95 & 1.23 & 5.09 & 7.3 & 1.10 \\
\hline
\end{tabular}

Table 4. Fit of the $e^{+} e^{-} \rightarrow h h(\rightarrow b \bar{b} b \bar{b}) \nu \bar{\nu}$ rate (see eq. (6.24)) at CLIC with $\sqrt{s}=3 \mathrm{TeV}$ in the various kinematical regions defined in eq. (6.23).
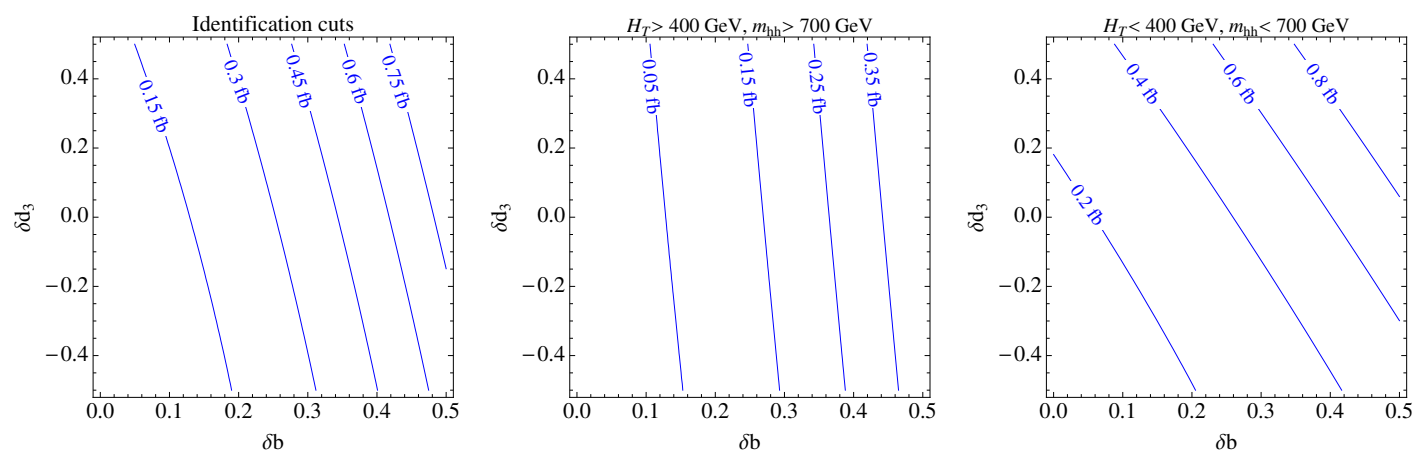

Figure 6. Contours of constant $e^{+} e^{-} \rightarrow h h(\rightarrow b \bar{b} b \bar{b}) \nu \bar{\nu}$ rate (see eq. (6.24)) for $\sqrt{s}=3 \mathrm{TeV}$ in the plane $\left(\delta_{b}, \delta_{d_{3}}\right)$. We set $a=1$ and $B R(h h \rightarrow b b \bar{b} \bar{b})=B R(h h \rightarrow b b \bar{b} \bar{b})_{S M}$.

using both $m_{h h}$ and $H_{T}$ gives a slightly better sensitivity. We thus consider the four independent kinematical regions

$$
\begin{aligned}
\text { I }: & m_{h h}>700 \mathrm{GeV} \text { and } H_{T}>400 \mathrm{GeV}, \\
\text { II }: & m_{h h}>700 \mathrm{GeV} \text { and } H_{T}<400 \mathrm{GeV}, \\
\text { III }: & m_{h h}<700 \mathrm{GeV} \text { and } H_{T}>400 \mathrm{GeV}, \\
\text { IV }: & m_{h h}<700 \mathrm{GeV} \text { and } H_{T}<400 \mathrm{GeV} .
\end{aligned}
$$

Our final results do not crucially depend on the specific choice of the cuts on $m_{h h}$ and $H_{T}$. One could in principle optimize them to obtain the best sensitivity on the parameters. We checked, however, that reasonable variations around the values adopted in eq. (6.23) result in small variations of the final results. For each of the kinematic regions (6.23), the signal rate $r \equiv \sigma\left(e^{+} e^{-} \rightarrow \nu \bar{\nu} h h\right) \times B R(h h \rightarrow b b \bar{b} \bar{b})$ can be parametrized as follows

$$
r=r_{S M} a^{4}\left(\frac{B R(b \bar{b})}{B R(b \bar{b})_{S M}}\right)^{2}\left(1+A_{r} \delta_{b}+B_{r} \delta_{d_{3}}+C_{r} \delta_{b} \delta_{d_{3}}+D_{r} \delta_{b}^{2}+E_{r} \delta_{d_{3}}^{2}\right)
$$

where $r_{S M}$ is the SM rate and $B R(b \bar{b})$ is the Higgs branching fraction to $b \bar{b}$. The values of the coefficients $A_{r}, B_{r}, C_{r}, D_{r}, E_{r}$ and of $r_{S M}$ are reported in table 4. Figure 6 shows the curves of constant rate in the plane $\left(\delta_{b}, \delta_{d_{3}}\right)$ for three choices of cuts: only the identification cuts of eqs. (6.2) and (6.22), identification cuts + region I, identification cuts + region IV. 


\begin{tabular}{|c|c|c|c|c|c|c|c|c|}
\hline \multirow{2}{*}{\multicolumn{2}{|c|}{$\begin{array}{c}\text { measured } \\
\delta_{b}\end{array}$}} & \multicolumn{7}{|c|}{$\bar{\delta}_{d_{3}}$} \\
\hline & & -0.5 & -0.3 & -0.1 & 0 & 0.1 & 0.3 & 0.5 \\
\hline \multirow{8}{*}{$\bar{\delta}_{b}$} & 0 & $-0.045_{-0.025}^{+0.060}$ & $0.015_{-0.040}^{+0.020}$ & $0.010_{-0.045}^{+0.070}$ & $0.00_{-0.05}^{+0.05}$ & $0.00_{-0.03}^{+0.03}$ & $0.00_{-0.03}^{+0.03}$ & $0.00_{-0.03}^{+0.03}$ \\
\hline & 0.01 & $-0.055_{-0.020}^{+0.070}$ & $0.030_{-0.045}^{+0.030}$ & $0.020_{-0.035}^{+0.080}$ & $0.015_{-0.035}^{+0.030}$ & $0.010_{-0.030}^{+0.020}$ & $0.010_{-0.025}^{+0.025}$ & $0.010_{-0.025}^{+0.025}$ \\
\hline & 0.02 & $0.02_{-0.035}^{+0.030}$ & $0.040_{-0.050}^{+0.040}$ & $0.025_{-0.020}^{+0.075}$ & $0.020_{-0.035}^{+0.030}$ & $0.020_{-0.025}^{+0.025}$ & $0.020_{-0.025}^{+0.025}$ & $0.020_{-0.025}^{+0.025}$ \\
\hline & 0.03 & $0.03_{-0.035}^{+0.030}$ & $0.050_{-0.050}^{+0.040}$ & $0.035_{-0.020}^{+0.030}$ & $0.030_{-0.025}^{+0.025}$ & $0.030_{-0.025}^{+0.025}$ & $0.030_{-0.025}^{+0.025}$ & $0.030_{-0.020}^{+0.020}$ \\
\hline & 0.05 & $0.05_{-0.035}^{+0.030}$ & $0.080_{-0.040}^{+0.020}$ & $0.055_{-0.020}^{+0.025}$ & $0.050_{-0.020}^{+0.025}$ & $0.050_{-0.025}^{+0.025}$ & $0.050_{-0.025}^{+0.025}$ & $0.050_{-0.020}^{+0.020}$ \\
\hline & 0.1 & $0.12_{-0.030}^{+0.025}$ & $0.10_{-0.02}^{+0.03}$ & $0.10_{-0.03}^{+0.03}$ & $0.10_{-0.03}^{+0.02}$ & $0.10_{-0.02}^{+0.02}$ & $0.10_{-0.02}^{+0.02}$ & $0.10_{-0.02}^{+0.02}$ \\
\hline & 0.3 & $0.30_{-0.02}^{+0.02}$ & $0.30_{-0.02}^{+0.02}$ & $0.30_{-0.02}^{+0.02}$ & $0.30_{-0.02}^{+0.02}$ & $0.30_{-0.02}^{+0.02}$ & $0.30_{-0.02}^{+0.02}$ & $0.30_{-0.02}^{+0.02}$ \\
\hline & 0.5 & $0.50_{-0.02}^{+0.02}$ & $0.50_{-0.02}^{+0.02}$ & $0.50_{-0.02}^{+0.02}$ & $0.50_{-0.02}^{+0.02}$ & $0.50_{-0.02}^{+0.02}$ & $0.50_{-0.02}^{+0.02}$ & $0.50_{-0.02}^{+0.02}$ \\
\hline
\end{tabular}

Table 5. Expected precision on $\delta_{b}$ for different true values $\bar{\delta}_{b}$ and $\bar{\delta}_{d_{3}}$ obtained at CLIC with $\sqrt{s}=3 \mathrm{TeV}$ and $L=1 \mathrm{ab}^{-1} /\left(a^{2} B R(b \bar{b}) / B R(b \bar{b})_{S M}\right)^{2}$ through $V V \rightarrow h h$ scattering.

In order to derive the expected sensitivity on $\delta_{b}$ and $\delta_{d_{3}}$, we construct a Poisson likelihood function (see eq. (6.14)) for each of the kinematical regions of eq. (6.23), and a global likelihood as the product of the individual ones. We assumed a flat prior on $\delta_{b}$ and $\delta_{d_{3}}$. Since $a$ and the branching ratio $B R(b \bar{b})$ appear in eq. (6.24) as overall factors, they can be conveniently absorbed by rescaling the integrated luminosity $L$ (note that, by the time the study of $V V \rightarrow h h$ will be feasible, both $a$ and $B R(b \bar{b})$ will be known precisely enough through single Higgs processes). The sensitivity on $\delta_{b}\left(\delta_{d_{3}}\right)$ is obtained by marginalizing the posterior probability over $\delta_{d_{3}}\left(\delta_{b}\right)$ and using the resulting single-parameter function to find the $68 \%$ probability interval on $\delta_{b}\left(\delta_{d_{3}}\right)$.

The results for a $3 \mathrm{TeV}$ linear collider with $L=1 \mathrm{ab}^{-1} /\left(a^{2} B R(b \bar{b}) / B R(b \bar{b})_{S M}\right)^{2}$ are shown in tables 5 and $6 .{ }^{20}$ For injected (true) values $\left(\bar{\delta}_{b}, \bar{\delta}_{d_{3}}\right)=(0,0)$ we find that the $68 \%$ error on $\delta_{d_{3}}$ is equal to $\sim 0.3$ (see table 6 ), which means that a measurement of the Higgs trilinear coupling in the SM should be possible with a precision of $\sim 30 \%$ with $L=1 \mathrm{ab}^{-1}$. This has to be compared with the $16 \%$ and $20 \%$ precisions reported respectively in ref. [123] and in the third paper of ref. [14-16] for $2 \mathrm{ab}^{-1}$ of integrated luminosity and unpolarized beams. For injected $\left(\bar{\delta}_{b}, \bar{\delta}_{d_{3}}\right)=(0,0)$ we also find that the precision attainable on $\delta_{b}$ with $L=1 \mathrm{ab}^{-1}$ is $\sim 5 \%$ (see table 5 ), which is compatible with the $3 \%$ recently reported for $L=2 \mathrm{ab}^{-1}$ by ref. [123].

The results of tables 5 and 6 have been obtained by considering $a, b$ and $d_{3}$ as independent parameters. Alternatively, by assuming them to be related as in eqs. (2.13) and (2.14) for the $\mathrm{SO}(5) / \mathrm{SO}(4)$ model MCHM4 (where $B R(b \bar{b})=B R(b \bar{b})_{S M}$ ), one can optimize the analysis to extract $\xi$. We do so by applying, besides the identification cuts of eqs. (6.2) and (6.22), a single cut on $H_{T}$ to isolate the energy growing behavior. Since we need to fit a single parameter, we select events with $H_{T}>400 \mathrm{GeV}$. The corresponding efficiencies

\footnotetext{
${ }^{20}$ Notice that for small $\delta_{b}$ and large and negative $\delta_{d_{3}}$, the central value of the measured parameter sometimes does not coincide with the true value. This is because in this limit, for our choice of integrated luminosity, the 2D likelihood can be largely non-gaussian and its marginalization over one parameter can lead to a shift of the central value of the second one.
} 


\begin{tabular}{|c|c|c|c|c|c|c|c|c|}
\hline \multirow{2}{*}{\multicolumn{2}{|c|}{$\begin{array}{l}\text { measured } \\
\qquad \delta_{d_{3}}\end{array}$}} & \multicolumn{7}{|c|}{$\bar{\delta}_{d_{3}}$} \\
\hline & & -0.5 & -0.3 & -0.1 & 0 & 0.1 & 0.3 & 0.5 \\
\hline \multirow{8}{*}{$\bar{\delta}_{b}$} & 0 & $-0.50_{-0.25}^{+0.35}$ & $-0.25_{-0.50}^{+0.20}$ & $0.00_{-0.40}^{+0.25}$ & $0.05_{-0.30}^{+0.30}$ & $0.10_{-0.20}^{+0.25}$ & $0.30_{-0.15}^{+0.20}$ & $0.50_{-0.15}^{+0.15}$ \\
\hline & 0.01 & $-0.45_{-0.30}^{+0.35}$ & $-0.20_{-0.55}^{+0.30}$ & $-0.05_{-0.30}^{+0.30}$ & $0.00_{-0.25}^{+0.25}$ & $0.10_{-0.20}^{+0.20}$ & $0.30_{-0.15}^{+0.15}$ & $0.50_{-0.15}^{+0.15}$ \\
\hline & 0.02 & $-0.35_{-0.35}^{+0.30}$ & $-0.25_{-0.60}^{+0.25}$ & $-0.10_{-0.30}^{+0.25}$ & $0.00_{-0.25}^{+0.20}$ & $0.10_{-0.20}^{+0.15}$ & $0.30_{-0.15}^{+0.15}$ & $0.50_{-0.15}^{+0.15}$ \\
\hline & 0.03 & $-0.40_{-0.35}^{+0.30}$ & $-0.25_{-0.70}^{+0.20}$ & $-0.10_{-0.25}^{+0.20}$ & $0.00_{-0.20}^{+0.15}$ & $0.10_{-0.20}^{+0.15}$ & $0.30_{-0.15}^{+0.15}$ & $0.50_{-0.15}^{+0.15}$ \\
\hline & 0.05 & $-0.55_{-0.40}^{+0.30}$ & $-0.30_{-0.30}^{+0.20}$ & $-0.10_{-0.20}^{+0.20}$ & $0.00_{-0.20}^{+0.15}$ & $0.10_{-0.15}^{+0.15}$ & $0.30_{-0.15}^{+0.15}$ & $0.50_{-0.10}^{+0.10}$ \\
\hline & 0.1 & $-0.50_{-0.25}^{+0.15}$ & $-0.30_{-0.20}^{+0.15}$ & $-0.10_{-0.20}^{+0.20}$ & $0.00_{-0.15}^{+0.15}$ & $0.10_{-0.15}^{+0.15}$ & $0.30_{-0.10}^{+0.10}$ & $0.50_{-0.10}^{+0.10}$ \\
\hline & 0.3 & $-0.50_{-0.15}^{+0.15}$ & $-0.30_{-0.15}^{+0.15}$ & $-0.10_{-0.10}^{+0.10}$ & $0.00_{-0.10}^{+0.10}$ & $0.10_{-0.10}^{+0.10}$ & $0.30_{-0.10}^{+0.10}$ & $0.50_{-0.10}^{+0.10}$ \\
\hline & 0.5 & $-0.50_{-0.10}^{+0.15}$ & $-0.30_{-0.10}^{+0.10}$ & $-0.10_{-0.10}^{+0.10}$ & $0.00_{-0.10}^{+0.10}$ & $0.10_{-0.10}^{+0.10}$ & $0.30_{-0.10}^{+0.10}$ & $0.50_{-0.10}^{+0.10}$ \\
\hline
\end{tabular}

Table 6. Expected precision on $\delta_{d_{3}}$ for different true values $\bar{\delta}_{b}$ and $\bar{\delta}_{d_{3}}$ obtained at CLIC with $\sqrt{s}=3 \mathrm{TeV}$ and $L=1 \mathrm{ab}^{-1} /\left(a^{2} B R(b \bar{b}) / B R(b \bar{b})_{S M}\right)^{2}$ through $V V \rightarrow h h$ scattering.

\begin{tabular}{|l|ccc|}
\hline & All $\times\left[H_{T}>400 \mathrm{GeV}\right]$ & No $\eta \times\left[H_{T}>400 \mathrm{GeV}\right]$ & No $\Delta R \times\left[H_{T}>400 \mathrm{GeV}\right]$ \\
\hline$\xi=0$ & $0.07=0.28 \times 0.24$ & $0.10=0.90 \times 0.11$ & $0.08=0.30 \times 0.26$ \\
$\xi=0.1$ & $0.15=0.35 \times 0.44$ & $0.20=0.89 \times 0.23$ & $0.18=0.39 \times 0.46$ \\
$\xi=0.5$ & $0.42=0.55 \times 0.77$ & $0.50=0.81 \times 0.62$ & $0.54=0.65 \times 0.83$ \\
\hline
\end{tabular}

Table 7. Efficiencies of the kinematic cuts imposed on the $e^{+} e^{-} \rightarrow \nu \bar{\nu} h h$ signal events to extract the parameter $\xi$ at CLIC with $\sqrt{s}=3 \mathrm{TeV}$. The format is $A=B \times C$, where $B$ is the efficiency for the identification cuts of eqs. (6.2) and (6.22), and $C$ is the efficiency of the cut $H_{T}>400 \mathrm{GeV}$ on the reconstructed Higgses.

are reported in table 7. Larger values of $\xi$ give larger efficiencies for the identification cuts, as mainly due to the stronger boost of the Higgses, as previously discussed. The signal rate can be parametrized in this case as follows

$$
r(\xi)=r_{S M}\left(1+A_{r} \xi+B_{r} \xi^{2}\right)
$$

The SM rate $r_{S M}$ and the coefficients $A_{r}, B_{r}$ are reported in table 8 . In order to estimate the sensitivity on $\xi$ that can be reached at CLIC, for any given true value $\bar{\xi}$ we construct a posterior probability (see eqs. (6.13) and (6.14)) by assuming a prior on $\xi$ which is flat in the theoretically allowed range $[0,1]$ and vanishing outside. The results are shown in table 9 for $L=1 \mathrm{ab}^{-1}$.

The results obtained in this section can be translated into an estimate of the sensitivity of CLIC on the scale of compositeness. In the presence of a shift in the Higgs couplings, $\delta_{h} \sim(v / f)^{2}$, the low-energy theory becomes strongly coupled at the scale $\Lambda=4 \pi f \sim$ $4 \pi v / \sqrt{\delta_{h}}$ unless New Physics states set in at a scale $m_{\rho}<\Lambda$, expectedly freezing the growth of the coupling at $g_{\rho} \sim m_{\rho} / f<4 \pi$. From tables 5 and 9 we conclude that the study of double-Higgs production at CLIC with $3 \mathrm{TeV}$ can lead to a sensitivity on $\Lambda$ of the 


\begin{tabular}{|r|ccc|}
\hline & $r_{S M}[\mathrm{ab}]$ & $A_{r}$ & $B_{r}$ \\
\hline All cuts & 15 & 11 & 106 \\
No $H_{T}$ & 63 & 4.1 & 28.3 \\
No $\eta$ & 23 & 10.5 & 76.9 \\
No $\Delta R$ & 17 & 11 & 118 \\
\hline
\end{tabular}

Table 8. Fit of the $e^{+} e^{-} \rightarrow h h(\rightarrow b \bar{b} b \bar{b}) \nu \bar{\nu}$ in the MCHM4 (see eq. (6.25)) at CLIC with $\sqrt{s}=$ $3 \mathrm{TeV}$. The numbers in the second row have been obtained by applying the whole set of kinematic cuts described in the text (eqs. (6.2), (6.22) and the cut on $H_{T}$ ), while each of the last three rows is obtained by removing one the cuts.

\begin{tabular}{|r|cccccc|}
\hline & \multicolumn{5}{|c|}{$\bar{\xi}$} \\
All cuts & 0 & 0.02 & 0.05 & 0.1 & 0.2 & 0.5 \\
\hline No $H_{T}$ & $0_{-0}^{+0.020}$ & $0.02_{-0.015}^{+0.015}$ & $0.05_{-0.015}^{+0.015}$ & $0.1_{-0.015}^{+0.015}$ & $0.2_{-0.015}^{+0.015}$ & $0.5_{-0.015}^{+0.010}$ \\
No $\eta$ & $0.02_{-0.020}^{+0.015}$ & $0.05_{-0.020}^{+0.020}$ & $0.1_{-0.015}^{+0.015}$ & $0.2_{-0.015}^{+0.015}$ & $0.5_{-0.015}^{+0.010}$ \\
No $\Delta R$ & $0_{-0}^{+0.015}$ & $0.02_{-0.015}^{+0.015}$ & $0.05_{-0.015}^{+0.015}$ & $0.1_{-0.015}^{+0.015}$ & $0.2_{-0.015}^{+0.010}$ & $0.5_{-0.010}^{+0.010}$ \\
\hline & $0_{-0}^{+0.020}$ & $0.02_{-0.015}^{+0.015}$ & $0.05_{-0.015}^{+0.015}$ & $0.1_{-0.015}^{+0.015}$ & $0.2_{-0.010}^{+0.010}$ & $0.5_{-0.010}^{+0.010}$ \\
\hline
\end{tabular}

Table 9. Expected $68 \%$ probability intervals on $\xi$ for different true values $\bar{\xi}$ obtained at CLIC with $\sqrt{s}=3 \mathrm{TeV}$ and $L=1 \mathrm{ab}^{-1}$ through $V V \rightarrow h h$ scattering.

order of $\sim 15-20 \mathrm{TeV}$ with an accumulated luminosity of $1 \mathrm{ab}^{-1}$. This has to be compared with sensitivities of the order of $\sim 10 \mathrm{TeV}$ and $\sim 30-40 \mathrm{TeV}$ expected from the study of single-Higgs processes respectively at the $14 \mathrm{TeV}$ LHC with $300 \mathrm{fb}^{-1}[92,93]$ and at the ILC with $250 \mathrm{fb}^{-1}$ of luminosity accumulated at $\sqrt{s}=250 \mathrm{GeV}$ plus another $500 \mathrm{fb}^{-1}$ at $\sqrt{s}=500 \mathrm{GeV}[13,124-126]$. Table 13 summarizes the reach on the compositeness scale at various experiments from the study of single and double Higgs processes.

\subsection{Double Higgs-strahlung}

The cross section for double Higgs production through $W W$ fusion drops as the energy of the collider is lowered: in the SM it goes from $1 \mathrm{fb}$ for $\sqrt{s}=3 \mathrm{TeV}$ down to $0.01 \mathrm{fb}$ for $\sqrt{s}=500 \mathrm{GeV}$. At such low energies one has to resort to other processes in order to measure the anomalous Higgs couplings $\delta_{b}$ and $\delta_{d_{3}}$. One possibility is double Higgs-strahlung (DHS), $e^{+} e^{-} \rightarrow h h Z$ [141-145]. The relevant Feynman diagrams are shown in figure 7, and the analytic expression of the differential cross section is known (see appendix B). Figure 8 shows the value of the total cross section as a function of the $e^{+} e^{-}$center-of-mass energy for some values of $\delta_{b}$ and $\delta_{d_{3}}$. For $\delta_{b}=0$ the cross section drops as $1 / s$ at high energy, while it asymptotically approaches a constant value for $\delta_{b} \neq 0$. This different high-energy behavior is due to the $e^{+} e^{-} \rightarrow h h Z_{L}$ amplitude and can be easily derived by using the 


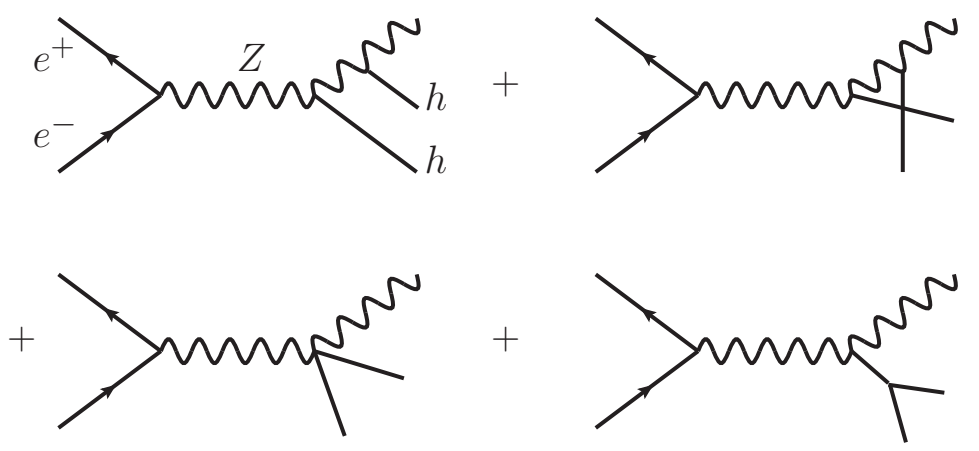

Figure 7. Diagrams contributing to double Higgs-strahlung at an $e^{+} e^{-}$collider. The two diagrams in the upper row are proportional to $a^{2}$, while the first and second diagrams in the lower row are proportional respectively to $b$ and $a d_{3}$.
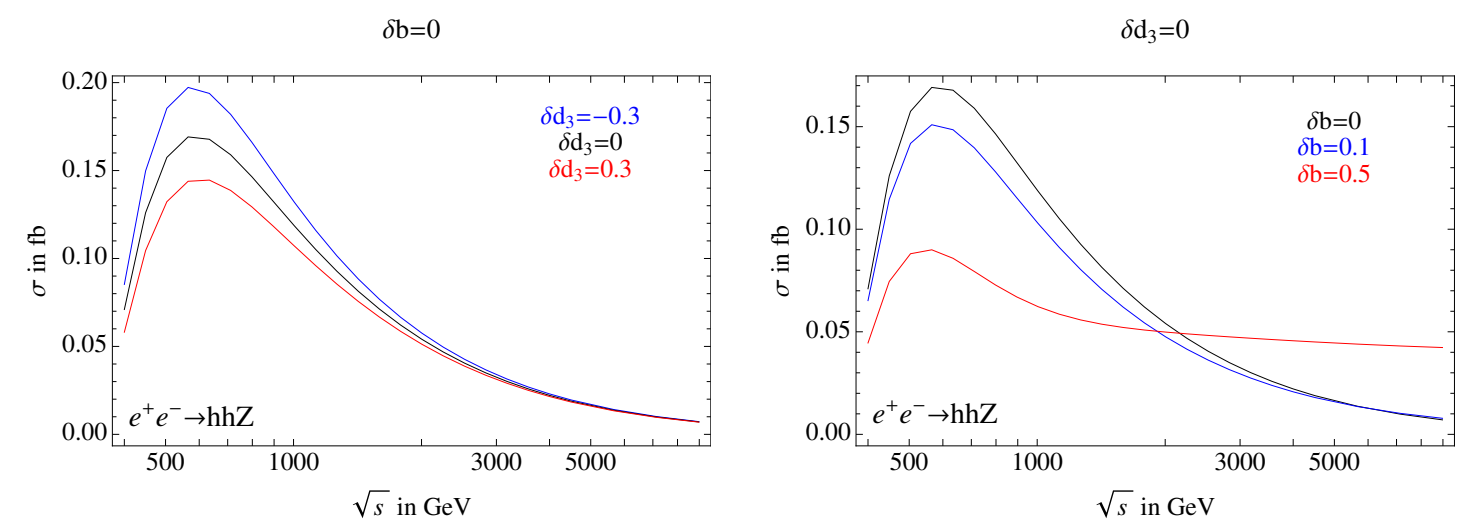

Figure 8. Total cross section of double Higgs-strahlung, $e^{+} e^{-} \rightarrow h h Z$, as a function of the c.o.m. energy for several values of the parameters $\delta_{b}$ and $\delta_{d_{3}}$.

\begin{tabular}{|c|cccccc|}
\hline$e^{+} e^{-} \rightarrow h h Z$ & $\sigma_{S M}[\mathrm{fb}]$ & $A$ & $B$ & $C$ & $D$ & $E$ \\
\hline $500 \mathrm{GeV}$ & 0.16 & -1.02 & -0.56 & 0.31 & 0.28 & 0.10 \\
$1 \mathrm{TeV}$ & 0.12 & -1.42 & -0.35 & 0.48 & 0.93 & 0.91 \\
$1 \mathrm{TeV}\left(m_{h h}<500\right)$ & 0.03 & -2.45 & -1.02 & 1.42 & 1.85 & 0.33 \\
$1 \mathrm{TeV}\left(m_{h h}>500\right)$ & 0.09 & -3.15 & -0.36 & 0.48 & 1.83 & 0.03 \\
\hline
\end{tabular}

Table 10. Parametrization of the double Higgsstrahlung $e^{+} e^{-} \rightarrow h h Z$ cross section (see eq. (6.18)) for various center-of-mass energies and cuts on the invariant mass of the two Higgses. The coefficients in the table have been computed by using the analytic expressions given in appendix B. Decay branching fractions and reconstruction efficiencies are not included.

equivalence theorem (see appendix B). Notice that for $m_{h}=125 \mathrm{GeV}$ the cross section is maximal between $500 \mathrm{GeV}$ and $1 \mathrm{TeV}$.

Before decaying the Higgs bosons, the DHS total cross section can also be parametrized as in eq. (6.18). By using the analytic expressions given in appendix B, we find the coefficients reported in table 10. In figure 9 we compare the invariant mass distributions 

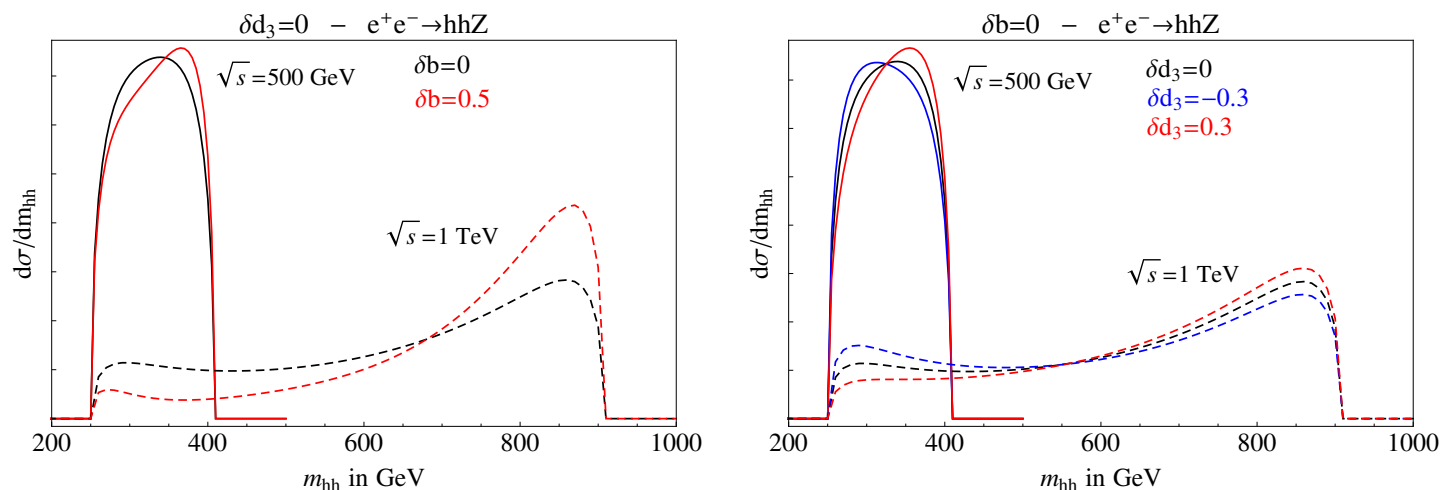

Figure 9. Differential cross section $d \sigma / d m_{h h}$ of double Higgsstrahlung at a linear collider with $\sqrt{s}=500 \mathrm{GeV}$ (solid lines) and $\sqrt{s}=1 \mathrm{TeV}$ (dashed lines), for several values of $\delta_{b}$ and $\delta_{d_{3}}$. All distributions have been normalized to unit area.

of the two Higgses at $\sqrt{s}=500 \mathrm{GeV}$ and $\sqrt{s}=1 \mathrm{TeV}$ for various values of the parameters. ${ }^{21}$

Our strategy to extract the anomalous couplings in this section differs in part from the one employed to analyze $W W$ scattering. In the case of DHS, at the energies we are interested in, the efficiency of the identification and reconstruction cuts is practically insensitive to the value of the Higgs couplings. The final rate can then be obtained by starting from the analytical expression of the cross section in terms of the parameters $\delta_{b}$ and $\delta_{d_{3}}$ given in eq. (6.18) and table 10, and rescaling the value of $\sigma_{S M}$ by an overall efficiency factor to include the decay branching fractions and the effect of kinematic cuts. We extracted such efficiency factor by generating a single sample of events corresponding to the SM choice of parameters. Such simplified approach fully exploits the analytic expression of the DHS cross section and greatly reduces the complexity of the Monte Carlo simulation.

The analysis of DHS turns out to be more difficult than the one of double Higgs production via $W W$ fusion due to the presence of non-negligible background processes. We focus on final states where both Higgses decay to $b \bar{b}$ and the $Z$ decays either hadronically or to a pair of charged leptons. We thus select events with 6 or more jets (and no lepton), or with 4 or more jets plus 2 opposite-charge leptons (electrons or muons). Jets and leptons are reconstructed according to the criteria defined in eqs. (6.2) and (6.3). Our selection ensures a full reconstruction of the momentum of the $Z$ boson in signal events and consequently a substantial reduction of background contamination. As a final discrimination we require at least 3 of the jets in the event to be $b$-tagged.

The reconstruction of the Higgs and $Z$ candidates proceeds as follows. In the case of events with 4 or more jets and 2 leptons, the $Z$ is reconstructed from the lepton pair, while the two Higgs candidates are identified as done in the case of $W W \rightarrow h h$, see section 6.3. We impose the following cut on the invariant mass of the lepton pair

$$
\left|m_{l l}-m_{Z}\right|<10 \mathrm{GeV},
$$

\footnotetext{
${ }^{21}$ The enhancement of the cross section at $m_{h h} \sim \sqrt{s}$ is due to the infrared singularity associated with the soft emission of a transversely-polarized $Z$ in the diagrams in the first row of figure 7 . The energy of the $Z$ boson, $E_{3}$, is related to the invariant mass of the two Higgses by the formula $m_{h h}^{2} / s=1-2 E_{3} / \sqrt{s}+m_{Z}^{2} / s$. In the limit $E_{3} \rightarrow 0$ it then follows $m_{h h} \rightarrow \sqrt{s}\left(1+O\left(m_{Z}^{2} / s\right)\right)$. See appendix B for more details.
} 


\begin{tabular}{|c|ccc|}
\hline & Energy & Efficiency & $r_{S M}[\mathrm{ab}]$ \\
\hline \multirow{5}{*}{$h h Z \rightarrow b \bar{b} b \bar{b} j j$} & $500 \mathrm{GeV}$ & 0.56 & 21 \\
& $1 \mathrm{TeV}-\mathrm{I}$ & 0.50 & 3.5 \\
& $1 \mathrm{TeV}-\mathrm{II}$ & 0.50 & 10.2 \\
& & & \\
& $500 \mathrm{GeV}$ & 0.58 & 2.2 \\
& $1 \mathrm{TeV}-\mathrm{I}$ & 0.56 & 0.34 \\
& $1 \mathrm{TeV}-\mathrm{II}$ & 0.56 & 1.0 \\
\hline
\end{tabular}

Table 11. Efficiencies for the identification of the Higgs and $Z$ candidates in the DHS signal at $500 \mathrm{GeV}$ and $1 \mathrm{TeV}$ (for both regions I and II of eq. (6.29)) for the two final states discussed in the analysis. The variation of the efficiency with the parameters $\delta_{b}$ and $\delta_{d_{3}}$ is negligible. The last column reports the SM rate after the identification cuts, $r_{S M}$, as defined in eq. (6.24).

while the invariant masses of the Higgs candidates are required to satisfy eq. (6.22). Events which do not satisfy these cuts are rejected. In the case of fully hadronic events, the Higgs and $Z$ candidates are reconstructed from the six most energetic jets, $j_{1, \ldots 6}$, by identifying the pairing $\left(j_{1} j_{2}, j_{3} j_{4}, j_{5} j_{6}\right)$ that minimizes the $\chi^{2}$ function

$$
\left(m_{j_{1} j_{2}}-m_{h}\right)^{2}+\left(m_{j_{3} j_{4}}-m_{h}\right)^{2}+\left(m_{j_{5} j_{6}}-m_{Z}\right)^{2} .
$$

We focus only on pairings where at least three among the four jets $j_{1 \ldots 4}$ are $b$-tagged, discarding the other pairings. This implies the presence of at least $3 b$-tags in the decay products of the two reconstructed Higgs bosons. After the Higgs $\left(j_{1} j_{2}\right.$ and $\left.j_{3} j_{4}\right)$ and $Z$ $\left(j_{5} j_{6}\right)$ candidates have been reconstructed, we impose a cut

$$
\left|m_{j_{5} j_{6}}-m_{Z}\right|<10 \mathrm{GeV}
$$

on the invariant mass of the $Z$ candidate, and the cut of eq. (6.22) on the invariant mass of each of the two Higgs candidates. Events where these requirements are not fulfilled are rejected. This algorithm has a fake rate (i.e. the rate at which it reconstructs fake Higgs or $Z$ candidates) always below $5 \%$ in the case of fully-hadronic events, and even smaller in the case of events with two leptons. The identification efficiencies on the signal at $\sqrt{s}=500 \mathrm{GeV}$ and $1 \mathrm{TeV}$ are given in table 11 for each of the two final states under consideration. They are to a large extent constant upon variations of $\delta_{b}$ and $\delta_{d_{3}}$. The signal rate $r\left(\delta_{b}, \delta_{d_{3}}\right)$ can be thus parametrized as in eq. (6.24) with coefficients $A_{r}, B_{r}, C_{r}, D_{r}, E_{r}$ equal to the $A, B, C, D, E$ given in table 10 , and an overall factor $r_{S M}$ fully subsuming the reconstruction efficiency and the decay branching fraction.

At a center-of-mass energy of $500 \mathrm{GeV}$, the signal rate is mostly dominated by events at the kinematical threshold. Disentangling the effect of $\delta_{b}$ from that of $\delta_{d_{3}}$ by means of kinematic cuts does not seem possible (at least for reasonable values of integrated luminosity). A measurement of the total cross section gives nevertheless the possibility to constrain a combination of the two relevant parameters $\delta_{b}$ and $\delta_{d_{3}}$. At the higher center-of-mass energy $\sqrt{s}=1 \mathrm{TeV}$, a better determination of both parameters is possible by cutting on the 


\begin{tabular}{|c|ccll|}
\hline & \multicolumn{4}{|c|}{ Rate } \\
& scaling with $a$ & $500 \mathrm{GeV}$ & $1 \mathrm{TeV}-\mathrm{I}$ & $1 \mathrm{TeV}-\mathrm{II}$ \\
\hline$b \bar{b} b \bar{b} j j$ & $a^{0}$ & $r_{b}^{(0)}=20 \mathrm{ab}$ & $r_{b}^{(0)}=1.4 \mathrm{ab}$ & $r_{b}^{(0)}=3.1 \mathrm{ab}$ \\
$h b \bar{b} j j$ & $a^{2}$ & $r_{b}^{(1)}=5.5 \mathrm{ab}$ & $r_{b}^{(1)}=0.4 \mathrm{ab}$ & $r_{b}^{(1)}=0.3 \mathrm{ab}$ \\
$b \bar{b} b \bar{b} \ell \ell$ & $a^{0}$ & $r_{b}^{(0)}=0.2 \mathrm{ab}$ & $r_{b}^{(0)}=0.05 \mathrm{ab}$ & $r_{b}^{(0)}=0.01 \mathrm{ab}$ \\
\hline
\end{tabular}

Table 12. Rates after identification and reconstruction cuts for the backgrounds included in our double Higgs-strahlung analysis at $\sqrt{s}=500 \mathrm{GeV}$ and $1 \mathrm{TeV}$ (for both regions I and II of eq. (6.29)). The leptonic background $h b b \bar{b} \ell \ell$ is negligible.

invariant mass of the two Higgs bosons. We define the two kinematical regions

$$
\begin{array}{cl}
\text { I : } & m_{h h}>500 \mathrm{GeV}, \\
\text { II : } & m_{h h}<500 \mathrm{GeV} .
\end{array}
$$

The signal rate in each region and for each of the two final states (leptonic and fully hadronic) is obtained by multiplying the cross section by the decay branching fraction and an overall reconstruction efficiency. ${ }^{22}$ The value of the SM rate $r_{S M}$ for each of the event categories at $\sqrt{s}=500 \mathrm{GeV}$ and $1 \mathrm{TeV}$ is reported in table 11 .

As previously mentioned, a crucial difference between DHS and double Higgs production via vector boson fusion is the presence of backgrounds processes that cannot be neglected. They can be classified according to their scaling with the parameter $a$, which sets the strength of the $h V V$ coupling. The powers of $a$ thus control the number of external Higgs boson legs. Notice that up to effects of order $\Gamma / m$ the interference between amplitudes with a different number of on-shell Higgs boson legs is negligible. Under this assumption, each factor $a^{2}$ is accompanied by one power of the Higgs decay branching ratio to $b \bar{b}$. The total rate can thus be parametrized as follows:

$$
r_{\text {tot }}\left(\delta_{b}, \delta_{d_{3}}\right)=r_{b}^{(0)}+a^{2} \frac{B R(b \bar{b})}{B R(b \bar{b})_{S M}} r_{b}^{(1)}+a^{4}\left(\frac{B R(b \bar{b})}{B R(b \bar{b})_{S M}}\right)^{2} r\left(\delta_{b}, \delta_{d_{3}}\right),
$$

where $r^{(0)}$ and $r^{(1)}$ are the rates for background processes respectively with 0 and 1 Higgs boson. Simple inspection of eq. (6.30) shows that in this case our results will depend in a non-trivial way on three quantities: $a^{2}\left(B R(b \bar{b}) / B R(b \bar{b})_{S M}\right), \delta_{b}$ and $\delta_{d_{3}}$. The backgrounds included in our analysis are listed in table 12 , together with their rate after applying the same reconstruction algorithm adopted for the signal.

We derive the expected sensitivity on $\delta_{b}$ and $\delta_{d_{3}}$ by assuming a flat prior on these coupling shifts and constructing a Poissonian likelihood function for each event category:

\footnotetext{
${ }^{22}$ Notice that in order for this procedure to be accurate it is crucial that the fake-rate of our algorithm for the reconstruction of the Higgs and $Z$ candidates is very small. If this was not the case, the measured invariant mass distribution of the Higgs pair could be affected by the reconstruction and this would invalidate our procedure.
} 

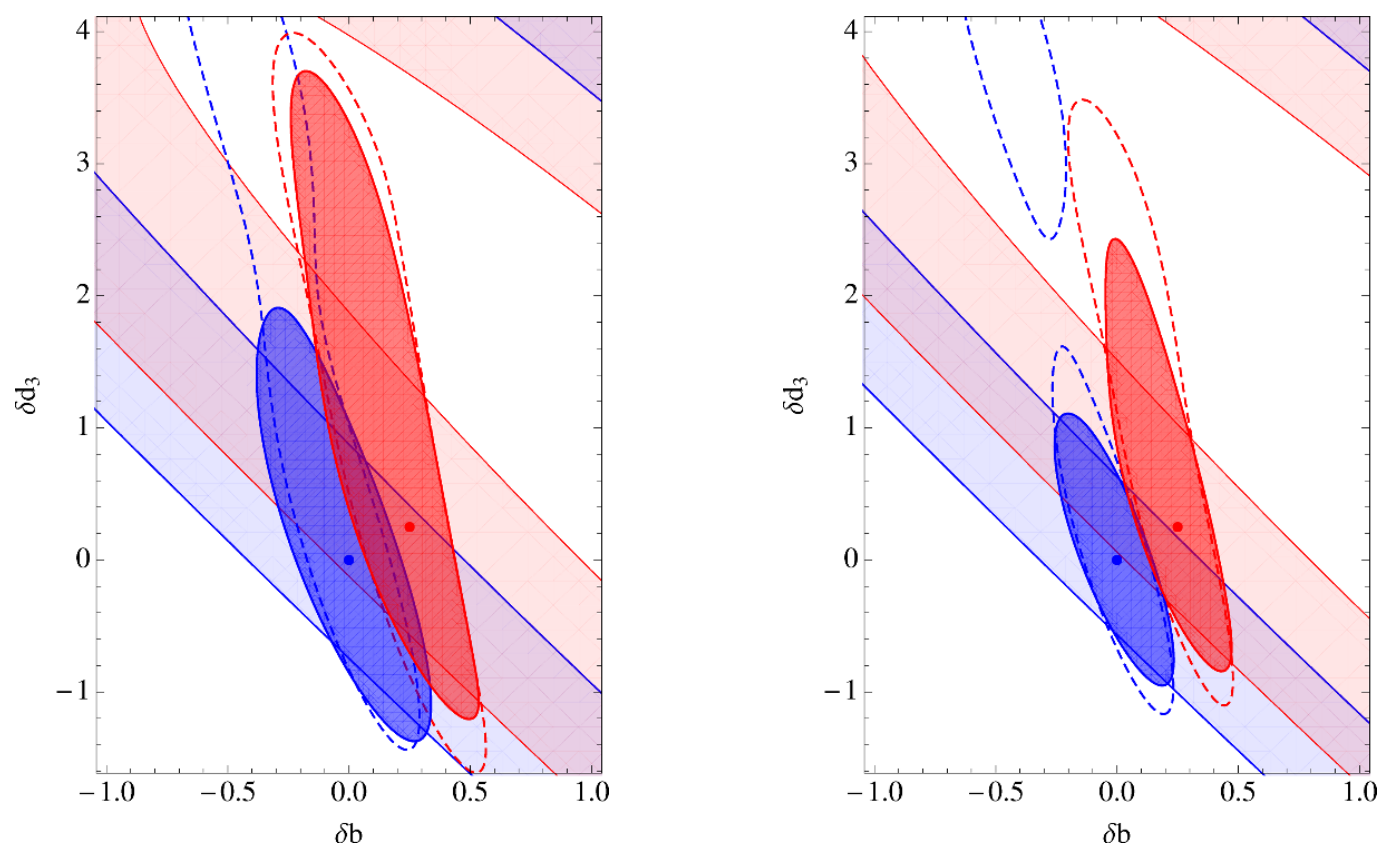

Figure 10. Regions of $68 \%$ probability in the plane $\left(\delta_{b}, \delta_{d_{3}}\right)$ obtained from the analysis of double Higgs-strahlung at various collider energies. Blue (red) shapes and contours are relative to the case of injected values $\bar{\delta}_{b}=0, \bar{\delta}_{d_{3}}=0\left(\bar{\delta}_{b}=0.25, \bar{\delta}_{d_{3}}=0.25\right)$. Lighter shaded bands: $500 \mathrm{GeV}$; Dashed contours: $1 \mathrm{TeV}$; Darker shaded regions: $500 \mathrm{GeV}+1 \mathrm{TeV}$. The plots have been obtained by assuming an integrated luminosity $L=1 \mathrm{ab}^{-1}$ and setting $a^{2}\left(B R(b \bar{b}) / B R(b \bar{b})_{S M}\right)=0.81$ (left plot) and $a^{2}\left(B R(b \bar{b}) / B R(b \bar{b})_{S M}\right)=1$ (right plot).

two categories for a $\sqrt{s}=500 \mathrm{GeV}$ collider (the leptonic and fully hadronic final states); four categories for a $\sqrt{s}=1 \mathrm{TeV}$ collider (two kinematic regions for each of the two final states). In each case, the total likelihood is obtained by taking the product of the individual ones. In general, we find that the fully hadronic final states lead to a better sensitivity on the couplings than the leptonic ones. As a way to effectively take into account the systematic and theoretical uncertainties on the estimate of the background in our statistical analysis, we have rescaled all the background rates by a factor 1.5 compared to the MC predictions reported in table 12 .

Figure 10 shows the regions of $68 \%$ probability obtained in the plane $\left(\delta_{b}, \delta_{d_{3}}\right)$ with $L=$ $1 \mathrm{ab}^{-1}$ by setting $a^{2}\left(B R(b \bar{b}) / B R(b \bar{b})_{S M}\right)=0.81$ (left plot) and $a^{2}\left(B R(b \bar{b}) / B R(b \bar{b})_{S M}\right)=1$ (right plot). The various contours are relative to the following two benchmark points: $\left(\delta_{b}, \delta_{d_{3}}\right)=(0,0)$ (in blue), and $\left(\delta_{b}, \delta_{d_{3}}\right)=(0.25,0.25)$ (in red). The latter point is obtained in the $\mathrm{SO}(4) / \mathrm{SO}(5) \mathrm{MCHM} 5$ for $\xi=0.2$, see eqs. (2.13), (2.14). The bands in light (red and blue) color indicate the result obtainable by measuring just the total cross section at a $500 \mathrm{GeV}$ collider. The red and blue dashed curves show instead the precision achievable at a linear collider with $1 \mathrm{TeV}$ c.o.m. energy by exploiting the cut on $m_{h h}$. If measurements at both $500 \mathrm{GeV}$ and $1 \mathrm{TeV}$ c.o.m. energies are possible (each with an integrated luminosity $L=1 \mathrm{ab}^{-1}$ ), an even more accurate precision on the couplings can be reached. The corresponding 68\% regions are shown in darker (red and blue) color in figure 10. 
An estimate of the precision attainable on the Higgs trilinear coupling at the ILC has been recently derived in ref. [13] assuming $a=1, b=1$. It is found that for $\sqrt{s}=500 \mathrm{GeV}$ and with an integrated luminosity $L=500 \mathrm{fb}^{-1}, d_{3}$ can be measured with a precision of $104 \%$ through DHS. At $\sqrt{s}=1 \mathrm{TeV}$ with $L=1 \mathrm{ab}^{-1}$, ref. [13] cites a precision of $28 \%$ through $V V \rightarrow h h$ scattering, while DHS is found to be less powerful. This suggests that a substantial improvement of our results can be obtained by including double Higgs production via vector boson fusion at $\sqrt{s}=1 \mathrm{TeV}$ into the analysis. Another recent study appeared in ref. [146] whose approach is more similar to the one presented in this paper. The couplings $b$ and $d_{3}$ are extracted through the measurement of the total DHS cross section at $500 \mathrm{GeV}$ and $1 \mathrm{TeV}$ (with no cut on $m_{h h}$ applied), and on double Higgs production through $V V$ fusion at $1 \mathrm{TeV}$. In the case of DHS, all the final states $Z \rightarrow \ell \ell, \nu \bar{\nu}, j j$ were included. We find that, although we did not include $V V \rightarrow h h$ and the $\nu \bar{\nu}$ final state in DHS, our analysis gives a better precision on the couplings $d_{3}$ and $b$ for those benchmark points where a comparison can be performed.

\section{Discussion}

The observation of a resonance with a mass around $125 \mathrm{GeV}$ and properties remarkably compatible with those of the Standard Model Higgs boson makes the questions about the dynamics at the origin of electroweak symmetry breaking more pressing. The most relevant and urgent issue now facing us concerns the structure of the newly discovered (Higgs) scalar. Are there additional states accompanying it? Is it elementary or is it composite? Could this really be the first elementary scalar observed in Nature, or could it just be a bound state arising from some novel strong dynamics, like a $\pi$ or $\eta$ in QCD? The answer to these questions will have profound implications on our picture of fundamental physics. That is because of the hierarchy problem. Establishing, to the best of our experimental capability, that the Higgs boson is elementary, weakly coupled and solitary, would surely be shocking, but it may well start a revolution in the basic concepts of quantum mechanics and spacetime. If instead deviations from the SM will emerge in the dynamics of the Higgs, we will have to use them as a diagnostic tool of the underlying dynamics. A crucial part of this program is the identification of the smoking guns of compositeness in Higgs dynamics. Moreover, along this basic question there are more specific ones we can ask, related to the symmetry properties of the new state. For instance, it will be essential to establish whether the new scalar is indeed "a Higgs" fitting into an SU(2) doublet and not some exotic impostor, like for instance a pseudo-dilaton. Although there is really no strong theoretical motivation for such an alternative, and so far the data disfavor it, it remains a logical possibility that can be tested and possibly ruled out. A perhaps more interesting question is whether the Higgs particle is just an ordinary composite, like a $\sigma$, or whether it is a pseudo-Nambu-Goldstone boson, like the $\pi$. The answer to this question will give us important clues on the UV completion of the electroweak breaking dynamics.

It is well known that in a fully natural theory of electroweak symmetry breaking the Higgs couplings must deviate, even in a significant way, from the predictions of the Standard Model. Note, however, that past and current experiments already put stringent constraints 
on these deviations, specifically on the single-Higgs $h V V$ coupling $a$, while the quadratic $h^{2} V V$ coupling $b$ and the Higgs cubic self-coupling $d_{3}$ so far remain unconstrained. Thanks to finally precise knowledge of the Higgs mass and to a more accurate measurement of the $W$ mass, the Higgs coupling $a$ is now constrained to lie with $95 \%$ probability in the interval $0.98 \leq a^{2} \leq 1.12$ under the assumption of no further contribution from New Physics. The tension with the theoretically motivated range $a<1$ can be slightly lifted by including an extra positive contribution to $\Delta \hat{T}$ in the electroweak fit, as it can arise in explicit models. Assuming $\Delta \hat{T}=+1.5 \times 10^{-3}$ leads to an interval $0.70 \leq a^{2} \leq 0.92$. Important constraints are also set by direct searches for spin-1 resonances at the LHC, which start to exclude interesting portions of the parameter space. Direct Higgs coupling measurements at the LHC, on the other hand, still have a limited precision but they are expected to reach a $\sim 5 \%$ resolution on $a$ at the $14 \mathrm{TeV}$ LHC.

In this paper we laid down a strategy to infer information about the scale and the nature of the dynamics behind EWSB through a precise measurement of the Higgs couplings. Observing a shift in the Higgs couplings of order $\delta_{h}$ in single-Higgs processes, together with the absence of any other new degree of freedom below a scale $M$, puts a qualitative lower bound on the strength of the interaction within the New Physics sector, $g_{\rho}>\sqrt{\delta_{h}} \times M / v$. This could provide a first indirect evidence for strong dynamics at the origin of EWSB and a hint towards a composite nature of the Higgs boson. For instance, $O(10 \%)$ deviations without any new states below $2 \mathrm{TeV}$, would already correspond to a coupling exceeding all the SM interactions in strength. Qualitatively, an analogous lower bound on $g_{\rho}$ can be obtained from the observation of an enhanced amplitude for the scattering of massive gauge bosons. Indeed, as we point out in section 4, at an electron-positron collider, the precision of the measurements could in principle allow us to estimate the size of subleading terms in the growth of the amplitude, thus providing a stronger indirect bound on the strength of the coupling.

Multi-Higgs production can bring additional valuable information to characterize the strong sector, even if it does not provide stronger bounds on its coupling strength or its scale. Double Higgs production by vector boson fusion gives access to the linear and quadratic couplings of the Higgs to the electroweak gauge bosons. We established a universal relation among these couplings, valid at order $v^{2} / f^{2}$ (where the scale $f$ is defined by $f=m_{\rho} / g_{\rho}$ for a generic composite state and corresponds to the decay constant for a PNGB), that follows when the Higgs boson is part of an electroweak doublet. This is because a single operator of dimension- 6 controls the leading corrections to both scattering amplitudes and single Higgs couplings. Furthermore, we studied the corrections to this relation that arise at order $v^{4} / f^{4}$ from dimension- 8 operators and we demonstrated that they can distinguish scenarios with a PNGB Higgs from those where the discovered boson is a generic light scalar resonance of the strong dynamics. The reason for this non-trivial result, is that, in the case of a PNGB Higgs, the non-linearly realized symmetry relates operators of different dimension.

We also emphasized the importance of a precise and energetic lepton collider such as CLIC to study the rare process of triple Higgs production through vector boson fusion, $V V \rightarrow h h h$. For a generic composite Higgs, the leading expected growing behaviour of 
the cross section below the scale of the resonances is $\sim v^{2} s^{2} / f^{8}$ and could in principle be observed provided $v^{2} / f^{2} \sim 0.1$. However we pointed out that for a PNGB Higgs based on cosets involving only doublets, in particular in the simplest cases of $\mathrm{SO}(5) / \mathrm{SO}(4)$ or $\mathrm{SO}(4,1) / \mathrm{SO}(4)$, this leading term exactly cancels. This cancellation is a simple consequence of the homogeneity and of the grading symmetry of such cosets, but in the effective lagrangian it corresponds to a more obscure correlation among the coefficients of operators of dimension 6 and dimension 8. This is the same correlation we mentioned before. The observation, or lack thereof, of a visible rate for $V V \rightarrow h h h$ could then play a relevant role in the reconstruction of the underlying theory.

We presented a quantitative analysis of vector boson scattering and double Higgs production, both through vector boson fusion and double Higgs-strahlung, at the ILC and CLIC for two different center-of-mass energies. Focusing on $V V \rightarrow V V$ scattering processes and using a simple cut-and-count analysis, we found that a $\sqrt{s}=500 \mathrm{GeV}$ linear collider with an integrated luminosity of $1 \mathrm{ab}^{-1}$ is only sensitive to large deviations of the coupling $a$ from its SM value, $\Delta a^{2} \sim 0.5$ (see footnote 18 for the definition of sensitivity used in this paper). A $3 \mathrm{TeV}$ linear collider with the same luminosity, on the other hand, is sensitive to shifts in $a^{2}$ larger than $\sim 0.2$. Double Higgs production depends both on $a$ and on the couplings $b$ and $d_{3}$. Its cross section can be conveniently expressed in terms of the two shifts $\delta_{b} \equiv 1-b / a^{2}$ and $\delta_{d_{3}} \equiv 1-d_{3} / a$, while the parameter $a$ enters as a simple overall rescaling which can be absorbed in the value of the luminosity (note that, at the time of the studies we are proposing, $a$ will be known with good accuracy thanks to single Higgs processes, hence $\delta_{b}$ and $\delta_{d_{3}}$ will really measure the deviations in the $b$ and $d_{3}$ couplings). As it emerges clearly throughout our study, $\delta_{b}$ offers a more sensitive probe into the Higgs structure than the trilinear $\delta_{d_{3}}$. In the case of a $3 \mathrm{TeV}$ CLIC machine with $L=1 \mathrm{ab}^{-1}$, the study of $V V \rightarrow h h$ offers a sensitivity of about 0.05 on $\delta_{b}$ while that on $\delta_{d_{3}}$ is hardly better than 0.3. In a specific model like the Minimal Composite Higgs model the couplings $a, b$ and $d_{3}$ depend on the single parameter $\xi=(v / f)^{2}$. Through the study of $e^{+} e^{-} \rightarrow h h \nu \bar{\nu}$, a machine like CLIC with $\sqrt{s}=3 \mathrm{TeV}$ and $L=1 \mathrm{ab}^{-1}$ can reach a sensitivity as small as 0.02 on $\xi$. These sensitivities can be translated into an indirect reach on the cutoff scale $\Lambda \equiv 4 \pi f$, that is the mass scale of the resonances for the case where the underlying dynamics is maximally strong. We find a reach $\Lambda \sim 15-20 \mathrm{TeV}$, which should be compared with the reach $\Lambda \sim 30-40 \mathrm{TeV}$ expected through single-Higgs processes at the ILC with $250 \mathrm{fb}^{-1}$ of luminosity accumulated at $\sqrt{s}=250 \mathrm{GeV}$ plus another $500 \mathrm{fb}^{-1}$ at $\sqrt{s}=500 \mathrm{GeV}[13,124-126]$. Table 13 summarizes the values of $\Lambda$ which can be probed at various experiments through the study of single and double Higgs processes. Though the reach on $\Lambda$ seems remarkable, one should not forget that the measured value of the Higgs mass disfavors a maximally strong coupling [110]: new states are therefore expected significantly below $\Lambda$ with a mass around $m_{\rho} \sim \Lambda \times g_{\rho} /(4 \pi)$. Still, even in the case of a moderately strong sector $g_{\rho} \sim 3$, direct production of resonances at a high-energy hadron collider like the LHC with $\sqrt{s}=33 \mathrm{TeV}$ may not become competitive. Of course one must beware of these qualitative arguments, as the model's details often matter.

At lower energies the $e^{+} e^{-} \rightarrow h h \nu \bar{\nu}$ process is not effective to measure the couplings $b, d_{3}$, and one has to resort to double Higgs-strahlung, $e^{+} e^{-} \rightarrow h h Z$. At $500 \mathrm{GeV}$ centerof-mass energy, only a linear combination of the two couplings $\delta_{b}$ and $\delta_{d_{3}}$ can be extracted 


\begin{tabular}{|lrlll|}
\hline & & $\xi=(v / f)^{2}$ & $\Lambda=4 \pi f$ \\
\hline LHC & $14 \mathrm{TeV} \quad L=300 \mathrm{fb}^{-1}$ & 0.5 (double Higgs [28, 29]) & $4.5 \mathrm{TeV}$ \\
& & 0.1 (single Higgs [92, 93]) & $10 \mathrm{TeV}$ \\
ILC $\quad 250 \mathrm{GeV} \quad L=250 \mathrm{fb}^{-1}$ & $0.6-1.2 \times 10^{-2}$ (single Higgs [13, 124-126]) & $30-40 \mathrm{TeV}$ \\
& $+500 \mathrm{GeV} \quad L=500 \mathrm{fb}^{-1}$ & & \\
CLIC $\quad 3 \mathrm{TeV} \quad L=1 \mathrm{ab}^{-1}$ & $2-5 \times 10^{-2}$ (double Higgs [this work]) & $15-20 \mathrm{TeV}$ \\
CLIC $\quad 350 \mathrm{GeV} \quad L=500 \mathrm{fb}^{-1}$ & & \\
& $+1.4 \mathrm{TeV} \quad L=1.5 \mathrm{ab}^{-1}$ & $1.1-2.4 \times 10^{-3}$ (single Higgs [123]) & $60-90 \mathrm{TeV}$ \\
$+3.0 \mathrm{TeV} \quad L=2 \mathrm{ab}^{-1}$ & & \\
\hline
\end{tabular}

Table 13. Summary of the precision on $\xi$ (as defined in footnote 18) and the corresponding reach on the compositeness scale at various experiments from the study of single and double Higgs processes.

from a measurement of the total cross section. For $\sqrt{s}=1 \mathrm{TeV}$, on the other hand, it is possible to exploit the kinematical distribution of the final state to extract both couplings independently, even though with large uncertainties. The combined measurement of double Higgs-strahlung at both $500 \mathrm{GeV}$ and $1 \mathrm{TeV}$ allows us to obtain the sensitivity contours shown in figure 10, which again indicate that $\delta_{b}$ can be measured more precisely than $\delta_{d_{3}}$. For all those interested in the structure of the Higgs the message is then very clear. The parameter $b$ not only encodes more robust information than $d_{3}$ about the nature of $h$, whether an impostor, a composite or a PNGB, but it also affords better sensitivity.

In the absence of direct production of new particles at the LHC, precision measurements in the sector of the newly discovered Higgs boson can play a key role in the search for New Physics. The time has come to establish a clear strategy to extract the information on the origin of electroweak symmetry breaking encoded in the Higgs measurements and to pave the way for a future experimental program.

\section{Acknowledgments}

We would like to thank Daniele Del Re, Enrico Franco, Gian Giudice, Lucie Linssen, Markus Luty, Barbara Mele, Paolo Meridiani, Satoshi Mishima, Alexandra Oliveira, Rogerio Rosenfeld, Luca Silvestrini, Alessandro Strumia, Riccardo Torre, James Wells and Andrea Wulzer for useful discussions and comments. We are grateful to Philipp Roloff and Marc Thomson for discussions and clarifications about the CLIC detector simulation. We thank Ian Low for pointing out a typo in the first version of the paper. The work of C.G. and A.T. has been partly supported by the European Commission under the contract ERC advanced grant 226371 'MassTeV' and the contract PITN-GA-2009-237920 'UNILHC'. The work of D.P. is supported by the NSF Grant PHY-0855653. The work of R.R. and A.T. is sup- 
ported by the Swiss National Science Foundation under contracts No. 200020-138131 and No. 200022-126941. R.C. was partly supported by the ERC Advanced Grant No. 267985 Electroweak Symmetry Breaking, Flavour and Dark Matter: One Solution for Three Mysteries (DaMeSyFla).

\section{A Dimension-8 operators for strong scatterings}

At the dimension- 8 level, the following three operators can be constructed with two derivatives and six Higgs fields

$$
\begin{aligned}
& c_{r}^{\prime} \mathcal{O}_{r}^{\prime}=\frac{c_{r}^{\prime}}{f^{2}}|H|^{4}\left|D_{\mu} H\right|^{2} \quad=|H|^{2} c_{r}^{\prime} \mathcal{O}_{r}, \\
& c_{H}^{\prime} \mathcal{O}_{H}^{\prime}=\frac{c_{H}^{\prime}}{2 f^{2}}|H|^{2} \partial_{\mu}|H|^{2} \partial^{\mu}|H|^{2} \quad=|H|^{2} c_{H}^{\prime} \mathcal{O}_{H}, \\
& c_{T}^{\prime} \mathcal{O}_{T}^{\prime}=\frac{c_{T}^{\prime}}{2 f^{2}}|H|^{2}\left(H^{\dagger} \overleftrightarrow{D}_{\mu} H\right)\left(H^{\dagger} \overleftrightarrow{D}^{\mu} H\right)=|H|^{2} c_{T}^{\prime} \mathcal{O}_{T},
\end{aligned}
$$

which can be found by constructing all possible $\mathrm{SU}(2)_{L}$-invariant structures and using integration by parts and the identities

$$
\begin{gathered}
\sigma_{i j}^{A} \sigma_{h k}^{A}=2 \delta_{i k} \delta_{j h}-\delta_{i j} \delta_{h k}, \\
\sigma_{i j}^{2} \sigma_{h k}^{2}=-\delta_{i h} \delta_{j k}+\delta_{j h} \delta_{i k} .
\end{gathered}
$$

The operators of eq. (A.1) consist of the dimension-6 structures discussed in ref. [29] extended by two Higgs fields. Note that $\mathcal{O}_{T}^{\prime}$ violates custodial symmetry, in analogy to $\mathcal{O}_{T}$. At the dimension-8 level there is also the operator $O_{8}=-c_{8}\left(H^{\dagger} H\right)^{4} / f^{2}$ defined in eq. (2.10), which involves no derivative. The operator $\mathcal{O}_{r}$ can be redefined away by the following field redefinition

$$
H \rightarrow H+\alpha H \frac{|H|^{2}}{f^{2}}+\beta H \frac{|H|^{4}}{f^{4}}
$$

under which the kinetic term transforms as follows

$$
\left|D_{\mu} H\right|^{2} \rightarrow\left|D_{\mu} H\right|^{2}+\frac{2 \alpha}{f^{2}} \mathcal{O}_{r}+\frac{\alpha}{f^{2}} \mathcal{O}_{H}+\frac{\alpha^{2}+2 \beta}{f^{4}}\left(\mathcal{O}_{r}^{\prime}+2 \mathcal{O}_{H}^{\prime}\right),
$$

while the dimension- 6 operators give

$$
\mathcal{O}_{T} \rightarrow \mathcal{O}_{T}+\frac{5 \alpha}{f^{2}} O_{T}^{\prime}+\ldots, \quad O_{H} \rightarrow \mathcal{O}_{H}+\frac{8 \alpha}{f^{2}} \mathcal{O}_{H}^{\prime}+\ldots, \quad \mathcal{O}_{r} \rightarrow O_{r}+\frac{8 \alpha}{f^{2}} \mathcal{O}_{r}^{\prime}+\frac{2 \alpha}{f^{2}} \mathcal{O}_{H}^{\prime}+\ldots
$$

Hence by choosing $\alpha$ and $\beta$ appropriately both $\mathcal{O}_{r}$ and $\mathcal{O}_{r}^{\prime}$ can be redefined away. We thus remain with only one custodially-invariant operator: $\mathcal{O}_{H}^{\prime}$. 


\section{B Double Higgs-strahlung}

The differential cross section for double Higgsstrahlung can be expressed in term of the Dalitz variables $x_{i} \equiv 2 E_{i} / \sqrt{s}$ where $E_{1,2}$ are the energies of the two Higgses and $x_{3} \equiv$ $2 E_{3} / \sqrt{s}$ for the $Z$ boson $[143,144]$ :

$$
\frac{d \sigma}{d x_{1} d x_{2}}=\frac{G_{F}^{3} m_{Z}^{6}}{384 \sqrt{2} \pi^{3} s} \frac{1+\left(1-4 s_{W}^{2}\right)^{2}}{\left(1-\mu_{Z}\right)^{2}} \mathcal{A} .
$$

Here $\sqrt{s}$ is the collider center-of-mass energy. We define $\mu_{i} \equiv m_{i}^{2} / s, \mu_{i j} \equiv \mu_{i}-\mu_{j}$, $y_{i} \equiv 1-x_{i}$, so that $x_{3}=2-x_{1}-x_{2}$ follows by energy conservation. We have

$$
\mathcal{A}=\mathcal{A}_{0}^{2} f_{0}+\frac{a^{2}}{4 \mu_{Z}\left(y_{1}+\mu_{h Z}\right)}\left(\frac{a^{2} f_{1}}{y_{1}+\mu_{h Z}}+\frac{a^{2} f_{2}}{y_{2}+\mu_{h Z}}+2 \mu_{Z} \mathcal{A}_{0} f_{3}\right)+\left(y_{1} \leftrightarrow y_{2}\right)
$$

with

$$
\mathcal{A}_{0}=3 \frac{m_{h}^{2}}{m_{Z}^{2}} \frac{a d_{3}}{y_{3}-\mu_{h Z}}+\frac{2 a^{2}}{y_{1}+\mu_{h Z}}+\frac{2 a^{2}}{y_{2}+\mu_{h Z}}+\frac{b}{\mu_{Z}}
$$

and

$$
\begin{aligned}
f_{0}= & \frac{1}{8} \mu_{Z}\left(\left(y_{1}+y_{2}\right)^{2}+8 \mu_{Z}\right) \\
f_{1}= & \left(y_{1}-1\right)^{2}\left(\mu_{Z}-y_{1}\right)^{2}-4 \mu_{h} y_{1}\left(y_{1}+y_{1} \mu_{Z}-4 \mu_{Z}\right)+\mu_{Z}\left(\mu_{Z}-4 \mu_{h}\right)\left(1-4 \mu_{h}\right)-\mu_{Z}^{2}, \\
f_{2}= & \left(\mu_{Z}\left(y_{3}+\mu_{Z}-8 \mu_{h}\right)-\left(1+\mu_{Z}\right) y_{1} y_{2}\right)\left(1+y_{3}+2 \mu_{Z}\right) \\
& +y_{1} y_{2}\left(y_{1} y_{2}+1+\mu_{Z}^{2}+4 \mu_{h}\left(1+\mu_{Z}\right)\right)+4 \mu_{h} \mu_{Z}\left(1+\mu_{Z}+4 \mu_{h}\right)+\mu_{Z}^{2} \\
f_{3}= & y_{1}\left(y_{1}-1\right)\left(\mu_{Z}-y_{1}\right)-y_{2}\left(y_{1}+1\right)\left(y_{1}+\mu_{Z}\right)+2 \mu_{Z}\left(1+\mu_{Z}-4 \mu_{h}\right) .
\end{aligned}
$$

The kinematical boundaries of the phase space integration are defined by

$$
\left|2\left(1-x_{1}-x_{2}+2 \mu_{h}-\mu_{Z}\right)+x_{1} x_{2}\right| \leq \sqrt{x_{1}^{2}-4 \mu_{h}} \sqrt{x_{2}^{2}-4 \mu_{h}} .
$$

Using

$$
x_{1}=\frac{s+m_{h}^{2}-m_{23}^{2}}{s}, \quad x_{2}=\frac{m_{12}^{2}+m_{23}^{2}-m_{h}^{2}-m_{Z}^{2}}{s}
$$

and

$$
\frac{d \sigma}{d x_{1} d x_{2}}=s^{2} \frac{d \sigma}{d m_{12}^{2} d m_{23}^{2}},
$$

one can obtain the differential cross section as a function of $m_{12} \equiv m_{h h}$ and $m_{23} \equiv m_{h Z}$. Figure 9 in particular is derived by integrating over $m_{23}$ and varying $m_{h h}$.

It is interesting to analyze the enhancement of the differential cross section $d \sigma / d m_{h h}$ near the kinematic boundary $m_{h h} \simeq \sqrt{s}$. As mentioned in footnote 21 , the enhancement is due to the singularity associated with the soft emission of a transversely-polarized $Z$ boson in the diagrams in the first row of figure 7 . The leading singular behavior can be thus isolated by setting $d_{3}=0$, to switch off the Higgs trilinear coupling, and by fixing the 

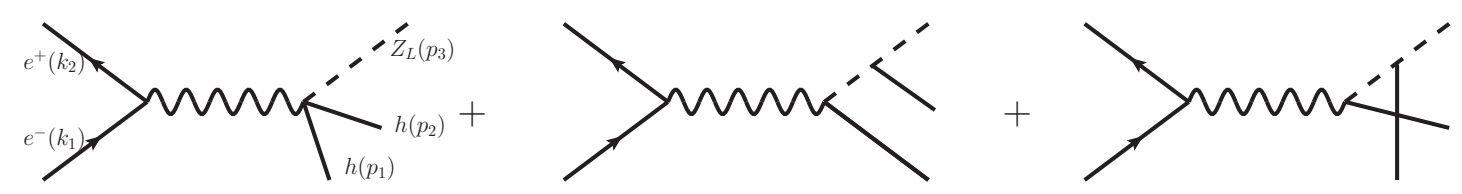

Figure 11. Diagrams contributing to the leading high-energy behavior of the double Higgsstrahlung cross section.

couplings $a$ and $b$ to their SM value $\left(\delta_{b}=0\right)$. It is useful to make a change of variables in eq. (B.1) from $\left(x_{1}, x_{2}\right)$ to $\left(r \equiv m_{h h}^{2} / s, x_{2}\right)$, where the energy of the $Z$ is related to the invariant mass of the two Higgses by

$$
r=1-x_{3}+\frac{m_{Z}^{2}}{s}
$$

By integrating $x_{2}$ over the interval $r+\epsilon \leq x_{2} \leq 1-\epsilon$ and expanding for $\epsilon=m_{Z}^{2} / s$ small, we obtain

$$
\frac{d \sigma}{d r} \simeq \frac{G_{F}^{3} m_{Z}^{6}\left[1+\left(1-4 s_{W}^{2}\right)^{2}\right]}{192 \sqrt{2} \pi^{3} s}\left[(1-r)+\frac{r}{1-r} \log (1-r)-\frac{r}{1-r} \log \epsilon\right]
$$

which shows the singularity at $r=1$. The logarithmic terms in the above formula follow from the collinear singularity also associated with the $Z$ emission. Notice that events with a final longitudinally-polarized $Z$ have no soft singularity. At very large c.o.m. energies the process $e^{+} e^{-} \rightarrow h h Z_{L}$ dominates the total cross section and its leading contribution, which is proportional to $\delta_{b}$, peaks at $m_{h h} / \sqrt{s} \sim 1 / \sqrt{7}$, see eq. (B.17). The left plot of figure 9 shows that for $\sqrt{s}=1 \mathrm{TeV}$ the values of the differential cross section near the kinematic edge increases when going from $\delta_{b}=0$ to $\delta_{b}=0.5$. This means that the contribution from transversely-polarized final $Z$ bosons is still large in this case, as also shown by the right plot of figure 8. We have checked that for larger c.o.m. energies (or, similarly, much larger values of $\delta_{b}$ ), the differential cross section $d \sigma / d m_{h h}$ eventually peaks at the intermediate values $m_{h h} / \sqrt{s} \sim 1 / \sqrt{7}$.

The high-energy limit of the double Higgs-strahlung total cross section can be easily calculated explicitly. In a gauge in which the equivalence theorem is manifest, the diagrams contributing to the leading high-energy behavior are those depicted in figure 11. The relevant vertices are found by expanding the Lagrangian of eq. (2.1)

$$
\Delta \mathcal{L}=a \frac{h}{v}\left(\partial_{\mu} \chi^{3}\right)^{2}-m_{Z}\left(2 a \frac{h}{v}+b \frac{h^{2}}{v^{2}}\right) \partial_{\mu} \chi^{3} Z^{\mu} .
$$

Neglecting the masses of the initial state leptons, as well as those of the Higgs and the $Z$ boson, the amplitude can be written as

$$
i \mathcal{A}\left(e^{+} e^{-} \rightarrow h h Z_{L}\right) \simeq\left(\sqrt{2} G_{F}\right)^{3 / 2} \frac{2 m_{Z}^{2}}{s}\left(b-a^{2}\right) \bar{v}\left(k_{2}\right) \not p_{3}\left(g_{V}-g_{A} \gamma^{5}\right) u\left(k_{2}\right),
$$

where $k_{1}$ and $k_{2}$ are the momenta of the incoming electron and positron, $p_{3}$ is the momentum of the outgoing $Z$ boson and $s$ is the center-of-mass energy. The vector and 
axial-vector couplings of the electron are given by

$$
g_{V}=-\frac{1}{2}+2 s_{W}^{2}, \quad g_{A}=-\frac{1}{2} .
$$

Squaring and averaging the amplitude over the initial spins one gets

$$
\overline{|\mathcal{A}|^{2}} \simeq 8 m_{Z}^{4}\left(\sqrt{2} G_{F}\right)^{3}\left(g_{V}^{2}+g_{A}^{2}\right)\left(b-a^{2}\right)^{2} \frac{p_{3} \cdot k_{1} p_{3} \cdot k_{2}}{s^{2}} .
$$

The total cross section is written as

$$
\sigma\left(e^{+} e^{-} \rightarrow h h Z_{L}\right)=\frac{1}{2} \times \frac{1}{2 s} \int \overline{|\mathcal{A}|^{2}} d \Phi^{(3)},
$$

where the extra 1/2 factor accounts for the two Higgs particles in the final state. The phase space integral can be done by using the recursive formula

$$
d \Phi^{(3)}\left(P ; p_{1}, p_{2}, p_{3}\right)=\int \frac{d p_{12}^{2}}{2 \pi} d \Phi^{(2)}\left(p_{12} ; p_{1}, p_{2}\right) d \Phi^{(2)}\left(P ; p_{12}, p_{3}\right) .
$$

Notice that $p_{12}^{2}$ is the invariant mass of the two Higgses. Since the amplitude in eq. (B.13) does not depend on the momenta of the two Higgs bosons, $p_{1}$ and $p_{2}$, the first phase space integral in eq. (B.15) is trivial and gives

$$
d \Phi^{(2)}\left(p_{12} ; p_{1}, p_{2}\right)=\frac{1}{8 \pi} .
$$

Taking into account the energy and angular dependence of the amplitude one obtains

$$
\frac{d \sigma}{d m_{h h}^{2}} \simeq \frac{\left(\sqrt{2} G_{F}\right)^{3}\left(g_{V}^{2}+g_{A}^{2}\right)}{1536 \pi^{3}} \frac{m_{Z}^{4}}{s}\left(b-a^{2}\right)^{2}\left(1-\frac{m_{h h}^{2}}{s}\right)^{3}
$$

and integrating over $0 \leq m_{h h}^{2} \leq s$ it follows

$$
\sigma \simeq \frac{\left(\sqrt{2} G_{F}\right)^{3}\left(g_{V}^{2}+g_{A}^{2}\right)}{6144 \pi^{3}} m_{Z}^{4}\left(b-a^{2}\right)^{2}=0.15 \mathrm{fb}\left(b-a^{2}\right)^{2} .
$$

Open Access. This article is distributed under the terms of the Creative Commons Attribution License (CC-BY 4.0), which permits any use, distribution and reproduction in any medium, provided the original author(s) and source are credited.

\section{References}

[1] ATLAS collaboration, Observation of a new particle in the search for the standard model Higgs boson with the ATLAS detector at the LHC, Phys. Lett. B 716 (2012) 1 [arXiv:1207.7214] [INSPIRE].

[2] CMS collaboration, Observation of a new boson at a mass of $125 \mathrm{GeV}$ with the CMS experiment at the LHC, Phys. Lett. B 716 (2012) 30 [arXiv:1207.7235] [INSPIRE].

[3] D.B. Kaplan and H. Georgi, $\mathrm{SU}(2) \times \mathrm{U}(1)$ breaking by vacuum misalignment, Phys. Lett. B 136 (1984) 183 [INSPIRE]. 
[4] S. Dimopoulos and J. Preskill, Massless composites with massive constituents, Nucl. Phys. B 199 (1982) 206 [INSPIRE].

[5] T. Banks, Constraints on $\mathrm{SU}(2) \times \mathrm{U}(1)$ breaking by vacuum misalignment, Nucl. Phys. $\mathrm{B}$ 243 (1984) 125 [inSPIRE].

[6] D.B. Kaplan, H. Georgi and S. Dimopoulos, Composite Higgs scalars, Phys. Lett. B 136 (1984) 187 [INSPIRE].

[7] H. Georgi, D.B. Kaplan and P. Galison, Calculation of the composite Higgs mass, Phys. Lett. B 143 (1984) 152 [INSPIRE].

[8] H. Georgi and D.B. Kaplan, Composite Higgs and custodial SU(2), Phys. Lett. B 145 (1984) 216 [INSPIRE].

[9] M.J. Dugan, H. Georgi and D.B. Kaplan, Anatomy of a composite Higgs model, Nucl. Phys. B 254 (1985) 299 [INSPIRE].

[10] A. De Roeck et al., From the LHC to future colliders, Eur. Phys. J. C 66 (2010) 525 [arXiv:0909.3240] [INSPIRE].

[11] E. Todesco and F. Zimmermann, Proceedings of EuCARD-AccNet-EuroLumi Workshop: the high-energy Large Hadron Collider, Malta, republic of Malta, 14-16 Oct 2010, arXiv:1111.7188 [INSPIRE].

[12] CMS collaboration, CMS at the high-energy frontier, talk given at the Open SYmposium European Strategy Preparatory Group, September 10-12, Krakow, Poland (2012), CERN-ATS-2012-237.clo (2012).

[13] H. Baer et al., The International Linear Collider technical design report - Volume 2: physics, arXiv:1306.6352 [INSPIRE].

[14] CLIC Physics Working Group collaboration, E. Accomando et al., Physics at the CLIC multi-TeV linear collider, hep-ph/0412251 [INSPIRE].

[15] L. Linssen, A. Miyamoto, M. Stanitzki and H. Weerts, Physics and detectors at CLIC: CLIC conceptual design report, arXiv: 1202.5940 [INSPIRE].

[16] P. Lebrun et al., The CLIC programme: towards a staged $e^{+} e^{-}$linear collider exploring the Terascale : CLIC conceptual design report, arXiv:1209.2543 [INSPIRE].

[17] V. Barger, T. Han, P. Langacker, B. McElrath and P. Zerwas, Effects of genuine dimension-six Higgs operators, Phys. Rev. D 67 (2003) 115001 [hep-ph/0301097] [INSPIRE].

[18] J. Baggeet al., The strongly interacting WW system: gold plated modes, Phys. Rev. D 49 (1994) 1246 [hep-ph/9306256] [INSPIRE].

[19] J. Bagger et al., CERN LHC analysis of the strongly interacting WW system: gold plated modes, Phys. Rev. D 52 (1995) 3878 [hep-ph/9504426] [inSPIRE].

[20] A. Ballestrero, G. Bevilacqua, D.B. Franzosi and E. Maina, How well can the LHC distinguish between the SM light Higgs scenario, a composite Higgs and the Higgsless case using VV scattering channels?, JHEP 11 (2009) 126 [arXiv:0909.3838] [INSPIRE].

[21] A. Ballestrero, G. Bevilacqua and E. Maina, A complete parton level analysis of boson-boson scattering and electroweak symmetry breaking in $l \nu+$ four jets production at the LHC, JHEP 05 (2009) 015 [arXiv: 0812.5084] [INSPIRE].

[22] CMS collaboration, P. Govoni, Study of $V V$-scattering processes as a probe of electroweak symmetry breaking, AIP Conf. Proc. 1078 (2009) 229 [INSPIRE]. 
[23] E. Accomando, A. Ballestrero, A. Belhouari and E. Maina, Boson fusion and Higgs production at the LHC in six fermion final states with one charged lepton pair, Phys. Rev. D 75 (2007) 113006 [hep-ph/0603167] [INSPIRE].

[24] J. Butterworth, B. Cox and J.R. Forshaw, WW scattering at the CERN LHC, Phys. Rev. D 65 (2002) 096014 [hep-ph/0201098] [INSPIRE].

[25] T. Han, D. Krohn, L.-T. Wang and W. Zhu, New physics signals in longitudinal gauge boson scattering at the LHC, JHEP 03 (2010) 082 [arXiv:0911.3656] [INSPIRE].

[26] D. Espriu and B. Yencho, Longitudinal WW scattering in light of the "Higgs boson" discovery, Phys. Rev. D 87 (2013) 055017 [arXiv:1212.4158] [INSPIRE].

[27] A. Freitas and J. Gainer, High energy $W W$ scattering at the LHC with the matrix element method, Phys. Rev. D 88 (2013) 017302 [arXiv:1212.3598] [InSPIRE].

[28] R. Contino, C. Grojean, M. Moretti, F. Piccinini and R. Rattazzi, Strong double Higgs production at the LHC, JHEP 05 (2010) 089 [arXiv:1002.1011] [INSPIRE].

[29] G. Giudice, C. Grojean, A. Pomarol and R. Rattazzi, The strongly-interacting light Higgs, JHEP 06 (2007) 045 [hep-ph/0703164] [INSPIRE].

[30] T. Appelquist and C.W. Bernard, Strongly interacting Higgs bosons, Phys. Rev. D 22 (1980) 200 [INSPIRE].

[31] A.C. Longhitano, Heavy Higgs bosons in the Weinberg-Salam model, Phys. Rev. D 22 (1980) 1166 [INSPIRE].

[32] A.C. Longhitano, Low-energy impact of a heavy Higgs boson sector, Nucl. Phys. B 188 (1981) 118 [INSPIRE].

[33] V. Koulovassilopoulos and R.S. Chivukula, The Phenomenology of a nonstandard Higgs boson in $W(L) W(L)$ scattering, Phys. Rev. D 50 (1994) 3218 [hep-ph/9312317] [INSPIRE].

[34] C. Burgess, J. Matias and M. Pospelov, A Higgs or not a Higgs? What to do if you discover a new scalar particle, Int. J. Mod. Phys. A 17 (2002) 1841 [hep-ph/9912459] [INSPIRE].

[35] B. Grinstein and M. Trott, A Higgs-Higgs bound state due to new physics at a TeV, Phys. Rev. D 76 (2007) 073002 [arXiv: 0704.1505] [INSPIRE].

[36] R. Alonso, M. Gavela, L. Merlo, S. Rigolin and J. Yepes, The effective chiral lagrangian for a light dynamical "Higgs Particle", Phys. Lett. B 722 (2013) 330 [Erratum ibid. B 726 (2013) 926] [arXiv: 1212.3305] [INSPIRE].

[37] E. Halyo, Technidilaton or Higgs?, Mod. Phys. Lett. A 8 (1993) 275 [InSPIRE].

[38] W.D. Goldberger, B. Grinstein and W. Skiba, Distinguishing the Higgs boson from the dilaton at the Large Hadron Collider, Phys. Rev. Lett. 100 (2008) 111802 [arXiv:0708.1463] [INSPIRE].

[39] L. Vecchi, Phenomenology of a light scalar: the dilaton, Phys. Rev. D 82 (2010) 076009 [arXiv: 1002.1721] [INSPIRE].

[40] B.A. Campbell, J. Ellis and K.A. Olive, Phenomenology and cosmology of an electroweak pseudo-dilaton and electroweak baryons, JHEP 03 (2012) 026 [arXiv:1111.4495] [INSPIRE].

[41] Z. Chacko, R. Franceschini and R.K. Mishra, Resonance at 125 GeV: Higgs or dilaton/radion?, JHEP 04 (2013) 015 [arXiv:1209.3259] [INSPIRE]. 
[42] B. Bellazzini, C. Csáki, J. Hubisz, J. Serra and J. Terning, A higgslike dilaton, Eur. Phys. J. C 73 (2013) 2333 [arXiv:1209.3299] [InSPIRE].

[43] M.S. Chanowitz and M.K. Gaillard, The TeV physics of strongly interacting $W$ 's and $Z$ 's, Nucl. Phys. B 261 (1985) 379 [InSPIRE].

[44] A. Wulzer, An equivalent gauge and the equivalence theorem, arXiv:1309.6055 [INSPIRE].

[45] R. Contino, D. Marzocca, D. Pappadopulo and R. Rattazzi, On the effect of resonances in composite Higgs phenomenology, JHEP 10 (2011) 081 [arXiv:1109.1570] [INSPIRE].

[46] M. Farina, C. Grojean and E. Salvioni, (Dys)Zphilia or a custodial breaking Higgs at the LHC, JHEP 07 (2012) 012 [arXiv:1205.0011] [INSPIRE].

[47] CMS Collaboration, Combination of standard model Higgs boson searches and measurements of the properties of the new boson with a mass near $125 \mathrm{GeV}$, CMS-PAS-HIG-13-005 (2013).

[48] ATLAS collaboration, Combined coupling measurements of the Higgs-like boson with the ATLAS detector using up to $25 \mathrm{fb}^{-1}$ of proton-proton collision data, ATLAS-CONF-2013-034 (2013).

[49] C. Burges and H.J. Schnitzer, Virtual effects of excited quarks as probes of a possible new hadronic mass scale, Nucl. Phys. B 228 (1983) 464 [INSPIRE].

[50] C.N. Leung, S. Love and S. Rao, Low-energy manifestations of a new interaction scale: operator analysis, Z. Phys. C 31 (1986) 433 [INSPIRE].

[51] W. Buchmüller and D. Wyler, Effective lagrangian analysis of new interactions and flavor conservation, Nucl. Phys. B 268 (1986) 621 [INSPIRE].

[52] R. Rattazzi, Anomalous interactions at the $Z^{0}$ pole, Z. Phys. C 40 (1988) 605 [INSPIRE].

[53] B. Grzadkowski, Z. Hioki, K. Ohkuma and J. Wudka, Probing anomalous top quark couplings induced by dimension-six operators at photon colliders, Nucl. Phys. B 689 (2004) 108 [hep-ph/0310159] [INSPIRE].

[54] C. Grojean, W. Skiba and J. Terning, Disguising the oblique parameters, Phys. Rev. D 73 (2006) 075008 [hep-ph/0602154] [INSPIRE].

[55] P.J. Fox, Z. Ligeti, M. Papucci, G. Perez and M.D. Schwartz, Deciphering top flavor violation at the LHC with B factories, Phys. Rev. D 78 (2008) 054008 [arXiv:0704.1482] [INSPIRE].

[56] J. Aguilar-Saavedra, A minimal set of top anomalous couplings, Nucl. Phys. B 812 (2009) 181 [arXiv: 0811.3842] [INSPIRE].

[57] J. Aguilar-Saavedra, A minimal set of top-Higgs anomalous couplings, Nucl. Phys. B 821 (2009) 215 [arXiv: 0904.2387] [InSPIRE].

[58] B. Grzadkowski, M. Iskrzynski, M. Misiak and J. Rosiek, Dimension-six terms in the standard model lagrangian, JHEP 10 (2010) 085 [arXiv: 1008.4884] [INSPIRE].

[59] R. Contino, M. Ghezzi, C. Grojean, M. Muhlleitner and M. Spira, Effective lagrangian for a light Higgs-like scalar, JHEP 07 (2013) 035 [arXiv: 1303.3876] [INSPIRE].

[60] J. Elias-Miro, J. Espinosa, E. Masso and A. Pomarol, Higgs windows to new physics through $D=6$ operators: constraints and one-loop anomalous dimensions, JHEP 11 (2013) 066 [arXiv: 1308.1879] [INSPIRE]. 
[61] K. Agashe, R. Contino and A. Pomarol, The minimal composite Higgs model, Nucl. Phys. B 719 (2005) 165 [hep-ph/0412089] [INSPIRE].

[62] R. Contino, L. Da Rold and A. Pomarol, Light custodians in natural composite Higgs models, Phys. Rev. D 75 (2007) 055014 [hep-ph/0612048] [INSPIRE].

[63] M. Baak et al., The electroweak fit of the standard model after the discovery of a new boson at the LHC, Eur. Phys. J. C 72 (2012) 2205 [arXiv:1209.2716] [InSPIRE].

[64] J. Erler, Tests of the electroweak standard model, arXiv:1209.3324 [INSPIRE].

[65] B. Batell, S. Gori and L.-T. Wang, Higgs couplings and precision electroweak data, JHEP 01 (2013) 139 [arXiv: 1209.6382] [inSPIRE].

[66] M. Ciuchini, E. Franco, S. Mishima and L. Silvestrini, Electroweak precision observables, new physics and the nature of a 126 GeV Higgs boson, JHEP 08 (2013) 106 [arXiv: 1306.4644] [INSPIRE].

[67] ALEPH, DELPHI, L3, OPAL, SLD, LEP Electroweak Working Group, SLD Electroweak Group, Sld Heavy Flavour Group, S. Schael et al., Precision electroweak measurements on the $Z$ resonance, Phys. Rept. 427 (2006) 257 [hep-ex/0509008] [INSPIRE].

[68] CDF, D0 collaboration, T.E.W. Group, 2012 update of the combination of CDF and D0 results for the mass of the $W$ boson, arXiv:1204.0042 [INSPIRE].

[69] R. Barbieri, B. Bellazzini, V.S. Rychkov and A. Varagnolo, The Higgs boson from an extended symmetry, Phys. Rev. D 76 (2007) 115008 [arXiv:0706.0432] [INSPIRE].

[70] M.E. Peskin and T. Takeuchi, Estimation of oblique electroweak corrections, Phys. Rev. D 46 (1992) 381 [INSPIRE].

[71] R. Barbieri, A. Pomarol, R. Rattazzi and A. Strumia, Electroweak symmetry breaking after LEP-1 and LEP-2, Nucl. Phys. B 703 (2004) 127 [hep-ph/0405040] [InSPIRE].

[72] I. Low, R. Rattazzi and A. Vichi, Theoretical constraints on the Higgs effective couplings, JHEP 04 (2010) 126 [arXiv:0907.5413] [INSPIRE].

[73] A. Falkowski, S. Rychkov and A. Urbano, What if the Higgs couplings to $W$ and $Z$ bosons are larger than in the standard model?, JHEP 04 (2012) 073 [arXiv:1202.1532] [INSPIRE].

[74] M. Golden and L. Randall, Radiative corrections to electroweak parameters in technicolor theories, Nucl. Phys. B 361 (1991) 3 [InSPIRE].

[75] R. Barbieri, G. Isidori and D. Pappadopulo, Composite fermions in electroweak symmetry breaking, JHEP 02 (2009) 029 [arXiv:0811.2888] [INSPIRE].

[76] C. Grojean, O. Matsedonskyi and G. Panico, Light top partners and precision physics, JHEP 10 (2013) 160 [arXiv:1306.4655] [INSPIRE].

[77] A. Azatov, R. Contino, A. Di Iura and J. Galloway, New prospects for Higgs compositeness in $h \rightarrow Z \gamma$, Phys. Rev. D 88 (2013) 075019 [arXiv:1308.2676] [InSPIRE].

[78] M.S. Carena, E. Ponton, J. Santiago and C.E. Wagner, Light Kaluza Klein states in Randall-Sundrum models with custodial SU(2), Nucl. Phys. B 759 (2006) 202 [hep-ph/0607106] [INSPIRE].

[79] P. Lodone, Vector-like quarks in a 'composite' Higgs model, JHEP 12 (2008) 029 [arXiv:0806.1472] [INSPIRE]. 
[80] M. Gillioz, A light composite Higgs boson facing electroweak precision tests, Phys. Rev. D 80 (2009) 055003 [arXiv:0806.3450] [InSPIRE].

[81] A. Pomarol and J. Serra, Top quark compositeness: feasibility and implications, Phys. Rev. D 78 (2008) 074026 [arXiv:0806.3247] [InSPIRE].

[82] A. Freitas and Y.-C. Huang, Electroweak two-loop corrections to $\sin ^{2} \theta_{\text {eff }}^{b \bar{b}}$ and $R_{b}$ using numerical Mellin-Barnes integrals, JHEP 08 (2012) 050 [Erratum ibid. 1305 (2013) 074] [arXiv: 1205.0299] [INSPIRE].

[83] D. Guadagnoli and G. Isidori, $B R\left(B_{s} \rightarrow \mu^{+} \mu^{-}\right)$as an electroweak precision test, Phys. Lett. B 724 (2013) 63 [arXiv:1302.3909] [INSPIRE].

[84] CDF Collaboration, D0 collaboration, T. Aaltonen et al., Combination of the top-quark mass measurements from the Tevatron collider, Phys. Rev. D 86 (2012) 092003 [arXiv:1207.1069] [INSPIRE].

[85] S. Alekhin, A. Djouadi and S. Moch, The top quark and Higgs boson masses and the stability of the electroweak vacuum, Phys. Lett. B 716 (2012) 214 [arXiv:1207.0980] [INSPIRE].

[86] T. Corbett, O.J.P. Eboli, J. Gonzalez-Fraile and M.C. Gonzalez-Garcia, Robust determination of the Higgs couplings: power to the data, Phys. Rev. D 87 (2013) 015022 [arXiv:1211.4580] [INSPIRE].

[87] A. Azatov and J. Galloway, Electroweak symmetry breaking and the Higgs boson: confronting theories at colliders, Int. J. Mod. Phys. A 28 (2013) 1330004 [arXiv:1212.1380] [INSPIRE].

[88] A. Falkowski, F. Riva and A. Urbano, Higgs at last, JHEP 11 (2013) 111 [arXiv:1303.1812] [INSPIRE].

[89] P.P. Giardino, K. Kannike, I. Masina, M. Raidal and A. Strumia, The universal Higgs fit, arXiv: 1303.3570 [INSPIRE].

[90] J. Ellis and T. You, Updated global analysis of Higgs couplings, JHEP 06 (2013) 103 [arXiv:1303.3879] [INSPIRE].

[91] A. Pomarol and F. Riva, Towards the ultimate SM fit to close in on Higgs physics, arXiv: 1308.2803 [INSPIRE].

[92] CMS collaboration, CMS at the high-energy frontier. Contribution to the update of the European Strategy for Particle Physics, CMS-NOTE-2012-006 (2012).

[93] ATLAS collaboration, Physics at a high-luminosity LHC with ATLAS (Update), ATL-PHYS-PUB-2012-004 (2012).

[94] R. Barbieri, G. Isidori, V.S. Rychkov and E. Trincherini, Heavy vectors in Higgs-less models, Phys. Rev. D 78 (2008) 036012 [arXiv:0806.1624] [INSPIRE].

[95] R. Barbieri, A. Carcamo Hernandez, G. Corcella, R. Torre and E. Trincherini, Composite vectors at the Large Hadron Collider, JHEP 03 (2010) 068 [arXiv:0911.1942] [INSPIRE].

[96] A. Carcamo Hernandez and R. Torre, A 'composite' scalar-vector system at the LHC, Nucl. Phys. B 841 (2010) 188 [arXiv: 1005.3809] [INSPIRE].

[97] A. Falkowski, C. Grojean, A. Kaminska, S. Pokorski and A. Weiler, If no Higgs then what?, JHEP 11 (2011) 028 [arXiv:1108.1183] [InSPIRE].

[98] R. Contino, T. Kramer, M. Son and R. Sundrum, Warped/composite phenomenology simplified, JHEP 05 (2007) 074 [hep-ph/0612180] [INSPIRE]. 
[99] M. Redi and A. Weiler, Flavor and CP invariant composite Higgs models, JHEP 11 (2011) 108 [arXiv: 1106.6357] [INSPIRE].

[100] CMS collaboration, Search for leptonic decays of $W^{\prime}$ bosons in pp collisions at $\sqrt{s}=8 \mathrm{TeV}$, CMS-PAS-EXO-12-060 (2012).

[101] CMS collaboration, Search for resonances in the dilepton mass distribution in pp collisions at $\sqrt{s}=8 \mathrm{TeV}$, CMS-PAS-EXO-12-061 (2012).

[102] ATLAS collaboration, Search for high-mass dilepton resonances in $20 \mathrm{fb}^{-1}$ of pp collisions at $\sqrt{s}=8$ TeV with the ATLAS experiment, ATLAS-CONF-2013-017 (2013).

[103] CMS collaboration, Search for $W^{\prime} /$ technirho in $W Z$ using leptonic final states, CMS-PAS-EXO-12-025 (2012).

[104] ATLAS collaboration, Search for resonant $W Z \rightarrow 3 l \nu$ production in $\sqrt{s}=8 \mathrm{TeV} p p$ collisions with $13 \mathrm{fb}^{-1}$ at ATLAS, ATLAS-CONF-2013-015 (2013).

[105] CMS collaboration, Search for new resonances decaying to $W W \rightarrow l \nu q \bar{q}^{\prime}$ in the final state with a lepton, missing transverse energy and single reconstructed jet,

CMS-PAS-EXO-12-021 (2012).

[106] CMS collaboration, Search for a narrow spin-2 resonance decaying to $Z$ bosons in the semileptonic final state, CMS-PAS-EXO-12-022 (2012).

[107] CMS collaboration, Search for heavy resonances in the W/Z-tagged dijet mass spectrum in pp collisions at $8 \mathrm{TeV}$, CMS-PAS-EXO-12-024 (2012).

[108] G. Panico, M. Redi, A. Tesi and A. Wulzer, On the tuning and the mass of the composite Higgs, JHEP 03 (2013) 051 [arXiv: 1210.7114] [INSPIRE].

[109] A. De Simone, O. Matsedonskyi, R. Rattazzi and A. Wulzer, A first top partner hunter's guide, JHEP 04 (2013) 004 [arXiv:1211.5663] [INSPIRE].

[110] D. Pappadopulo, A. Thamm and R. Torre, A minimally tuned composite Higgs model from an extra dimension, JHEP 07 (2013) 058 [arXiv: 1303.3062] [INSPIRE].

[111] A. Azatov and J. Galloway, Light custodians and Higgs physics in composite models, Phys. Rev. D 85 (2012) 055013 [arXiv:1110.5646] [INSPIRE].

[112] C. Delaunay, C. Grojean and G. Perez, Modified Higgs physics from composite light flavors, JHEP 09 (2013) 090 [arXiv: 1303.5701] [INSPIRE].

[113] M. Montull, F. Riva, E. Salvioni and R. Torre, Higgs couplings in composite models, Phys. Rev. D 88 (2013) 095006 [arXiv:1308.0559] [INSPIRE].

[114] M.S. Chanowitz and W. Kilgore, Complementarity of resonant and nonresonant strong WW scattering at the LHC, Phys. Lett. B 322 (1994) 147 [hep-ph/9311336] [INSPIRE].

[115] M.S. Chanowitz and W.B. Kilgore, $W^{+} Z$ and $W^{+} \gamma^{*}$ backgrounds to strong $W^{+} W^{+}$ scattering at the LHC, Phys. Lett. B 347 (1995) 387 [hep-ph/9412275] [INSPIRE].

[116] M.S. Chanowitz, Strong WW scattering at the end of the 90's: theory and experimental prospects, hep-ph/9812215 [INSPIRE].

[117] R. Casalbuoni et al., Vector resonances from a strong electroweak sector at linear colliders, Z. Phys. C 60 (1993) 315 [hep-ph/9303201] [INSPIRE].

[118] W. Bernreuther and T. Schroder, Strongly interacting Higgs sector and $W$ pair production in $e^{+} e^{-}$collisions, Z. Phys. C 62 (1994) 615 [INSPIRE]. 
[119] D. Dominici, Tests for a strong electroweak sector at future $e^{+} e^{-}$high-energy colliders, Riv. Nuovo Cim. 20N11 (1997) 1 [Riv. Nuovo Cim. 20 (1997) 1] [hep-ph/9711385] [InSPIRE].

[120] P. Poulose, S. Rindani and L. Sehgal, Lepton spectra from $e^{+} e^{-} \rightarrow W^{+} W^{-}$in the BESS model, Phys. Lett. B 525 (2002) 71 [hep-ph/0111134] [InSPIRE].

[121] T.L. Barklow, Strong symmetry breaking at $e^{+} e^{-}$linear colliders, eConf $\mathbf{C} 010630$ (2001) E3067 [hep-ph/0112286] [inSPIRE].

[122] B. Ananthanarayan, M. Patra and P. Poulose, Probing strongly interacting $W$ 's at the ILC with polarized beams, JHEP 03 (2012) 060 [arXiv:1112.5020] [INSPIRE].

[123] CLIC Detector and Physics Study collaboration, H. Abramowicz et al., Physics at the CLIC $e^{+} e^{-}$Linear Collider - Input to the Snowmass process 2013, arXiv:1307.5288 [INSPIRE].

[124] S. Dutta, K. Hagiwara and Y. Matsumoto, Measuring the Higgs-vector boson couplings at linear $e^{+} e^{-}$collider, Phys. Rev. D 78 (2008) 115016 [arXiv:0808.0477] [InSPIRE].

[125] M.E. Peskin, Comparison of LHC and ILC capabilities for Higgs boson coupling measurements, arXiv:1207.2516 [INSPIRE].

[126] M. Klute, R. Lafaye, T. Plehn, M. Rauch and D. Zerwas, Measuring Higgs couplings at a linear collider, Europhys. Lett. 101 (2013) 51001 [arXiv:1301.1322] [INSPIRE].

[127] O. Matsedonskyi, G. Panico and A. Wulzer, Light top partners for a light composite Higgs, JHEP 01 (2013) 164 [arXiv:1204.6333] [InSPIRE].

[128] D. Marzocca, M. Serone and J. Shu, General composite Higgs models, JHEP 08 (2012) 013 [arXiv:1205.0770] [INSPIRE].

[129] A. Pomarol and F. Riva, The composite Higgs and light resonance connection, JHEP 08 (2012) 135 [arXiv: 1205.6434] [INSPIRE].

[130] G. Ferrera, J. Guasch, D. Lopez-Val and J. Solà, Triple Higgs boson production in the linear collider, Phys. Lett. B 659 (2008) 297 [arXiv:0707.3162] [InSPIRE].

[131] A. Belyaev, A. Oliveira, R. Rosenfeld and M.C. Thomas, Multi Higgs and vector boson production beyond the standard model, JHEP 05 (2013) 005 [arXiv:1212.3860] [INSPIRE].

[132] T. Binoth, S. Karg, N. Kauer and R. Ruckl, Multi-Higgs boson production in the standard model and beyond, Phys. Rev. D 74 (2006) 113008 [hep-ph/0608057] [INSPIRE].

[133] V.D. Barger, K.-m. Cheung, T. Han and R. Phillips, Probing strongly interacting electroweak dynamics through $W^{+} W^{-} / Z Z$ ratios at future $e^{+} e^{-}$colliders, Phys. Rev. D 52 (1995) 3815 [hep-ph/9501379] [INSPIRE].

[134] T. Han, Higgs boson and $W(L) W(L)$ scattering at $e^{-} e^{-}$colliders, Int. J. Mod. Phys. A 11 (1996) 1541 [hep-ph/9601393] [INSPIRE].

[135] E. Boos et al., Strongly interacting vector bosons at TeV $e^{+} e^{-}$linear colliders, Phys. Rev. D 57 (1998) 1553 [hep-ph/9708310] [INSPIRE].

[136] T. Han, Y.-P. Kuang and B. Zhang, Anomalous gauge couplings of the Higgs boson at high energy photon colliders, Phys. Rev. D 73 (2006) 055010 [hep-ph/0512193] [INSPIRE].

[137] V.D. Barger and T. Han, Double Higgs boson production via WW fusion in $\mathrm{TeV} e^{+} e^{-}$ collisions, Mod. Phys. Lett. A 5 (1990) 667 [INSPIRE]. 
[138] E. Asakawa, D. Harada, S. Kanemura, Y. Okada and K. Tsumura, Higgs boson pair production in new physics models at hadron, lepton and photon colliders, Phys. Rev. D 82 (2010) 115002 [arXiv: 1009.4670] [INSPIRE].

[139] J. Alwall, M. Herquet, F. Maltoni, O. Mattelaer and T. Stelzer, MadGraph 5: going beyond, JHEP 06 (2011) 128 [arXiv:1106.0522] [InSPIRE].

[140] W. Kilian, T. Ohl and J. Reuter, WHIZARD: simulating multi-particle processes at LHC and ILC, Eur. Phys. J. C 71 (2011) 1742 [arXiv:0708.4233] [InSPIRE].

[141] G. Gounaris, D. Schildknecht and F. Renard, Test of Higgs boson nature in $e^{+} e^{-} \rightarrow H H Z$, Phys. Lett. B 83 (1979) 191 [INSPIRE].

[142] V.D. Barger, T. Han and R. Phillips, Double Higgs boson Bremsstrahlung from $W$ and $Z$ bosons at supercolliders, Phys. Rev. D 38 (1988) 2766 [InSPIRE].

[143] A. Djouadi, H. Haber and P. Zerwas, Multiple production of MSSM neutral Higgs bosons at high-energy $e^{+} e^{-}$colliders, Phys. Lett. B 375 (1996) 203 [hep-ph/9602234] [INSPIRE].

[144] A. Djouadi, W. Kilian, M. Muhlleitner and P. Zerwas, Testing Higgs selfcouplings at $e^{+} e^{-}$ linear colliders, Eur. Phys. J. C 10 (1999) 27 [hep-ph/9903229] [InSPIRE].

[145] S.S. Biswal et al., Role of polarization in probing anomalous gauge interactions of the Higgs boson, Phys. Rev. D 79 (2009) 035012 [arXiv:0809.0202] [INSPIRE].

[146] R. Killick, K. Kumar and H.E. Logan, Learning what the Higgs boson is mixed with, Phys. Rev. D 88 (2013) 033015 [arXiv: 1305.7236] [INSPIRE]. 